\title{
Cross Sections for Neutron-Producing Reactions Induced by 6- and 10-MeV Neutrons Incident on ${ }^{10} \mathrm{~B}$ and ${ }^{11} \mathrm{~B}$
}

\author{
M. Drosg \\ P. W. Lisowski \\ D. M. Drake \\ R. A. Hardekopf \\ S. M. Muellner**
}

LA--10665-MS

DE86 011734

"Consultant at Los Alamos. Department of Physics, University of Austria, Strudholgasse A-1090, lenat, AUSTRIA

"Department of Physics, University of Austria, Strudholgasse A-1090, lenat, AUSTRIA.

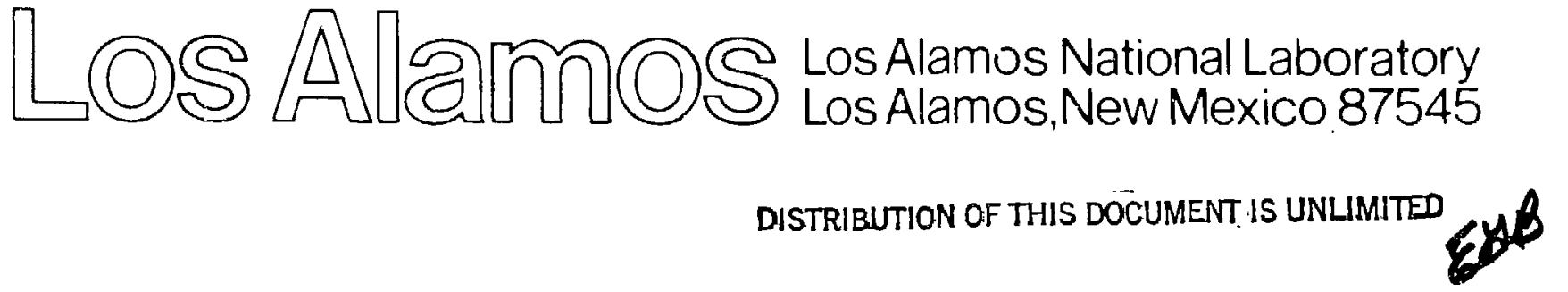




\title{
CROSS SECTIONS FOR NEUTRON-PRODUCING REACTIONS INDUCED
}

\section{BY 6- AND 10-MeV NEUTRONS INCIDENT ON ${ }^{10} \mathrm{~B}$ AND ${ }^{11} \mathrm{~B}$}

by

M. Drosg, P. W. Lisowski, D. M. Drake, R. A. Hardekopf, and M. Muellner

\begin{abstract}
Using the time-of-flight technique, we have measured the ${ }^{10} \mathrm{~B}$ and ${ }^{11_{B}}$ neutron emission spectra at incident neutron energies of 6.00 and $10.00 \mathrm{MeV}$ for angles between $20^{\circ}$ and $145^{\circ}$. Double differential cross sections and their integrated values have been extracted and are presented in tables and graphs. The integrated values (corrected for charged particle cross sections, if necessary) are in excellent agreement with measured total cross sections.
\end{abstract}

\section{INTRODUCTION}

Cross sactions for neutron-induced reactions on light nuclei below about $14 \mathrm{MeV}$ are important for fusion reactor design. Among these cross sections it is particularly difficult to measure nonelastic neutron cross sections because a monoenergetic neutron source of high yield is required to measure the resulting continuous spectra reliably. Between 8 and $14 \mathrm{MeV}$ only the ${ }^{1} \mathrm{H}(t, n){ }^{3} \mathrm{He}$ source has these required properties. Presently, the only installation that can supply bunched triton beams for fast neutron time-of-flight work are the Van de Graaffs of the Los Alamos National Laboratory. Consequently, double differential neutron cross sections of beryllium between 5.9 and $14.2 \mathrm{MeV}$, of lithium at 5.96 and $9.83 \mathrm{MeV}$, and of 
${ }^{6} \mathrm{Li},{ }^{7} \mathrm{Li},{ }^{10} \mathrm{~B},{ }^{11} \mathrm{~B}$, and carbon at $14.2 \mathrm{MeV}$ have been measured ${ }^{1-3}$ at the Los Alamos National Laboratory.

\section{EXPERIMENTAL DETAILS}

Bunched tritons from the tandem Van de Graaff at the Los Alamos National Laboratory were used to produce $6.00-\mathrm{MeV}$ and $10.00-\mathrm{MeV}$ neutrons by the ${ }^{1} H(t, n)^{3}$ He reaction with full width at half maximum (FWHM) energy spreads of $0.15 \mathrm{MeV}$ and $0.10 \mathrm{MeV}$, respectively. The rarget was a gas cell with an entrance foil of $5.3 \mathrm{mg} / \mathrm{cm}^{2}$ molybdenum and a beamstop of $0.05 \mathrm{~cm}$ gold. The target to sample distance was $14.0 \mathrm{~cm}$, the flight path $252.3 \mathrm{~cm}$. The ${ }^{10} \mathrm{~B}$ sample was a slightly irregularly shaped cylinder of $4 \mathrm{~cm}$ height, a purity of $95.5 \%$, and a mass of 1.80 moles. During the data analysis it was discovered that the spectra weie contaminated by peaks from elastic scattering from hydrogen. From the intensity of the peaks and the wellknown cross sections ${ }^{4}$ it was concluded that $0.31 \pm 0.03$ wto of the sample was hydrogen. Consequently all relevant spectra were corrected for this contamination. The ${ }^{11} \mathrm{~B}$ sample was a right-circular cylinder of about $3 \mathrm{~cm}$ height, $1.7 \mathrm{~cm}$ diameter, a purity of 90.58 , and a mass of 0.92 moles.

The general setup has been described before ${ }^{1,2}$, as well as the measurement of ti: efficiency of the NE213 neutron detector ${ }^{5}$ at the $0.3-\mathrm{MeV}$ bias as used in this experiment.

The advantages of using the ${ }^{1} \mathrm{H}(t, n){ }^{3} \mathrm{He}$ reaction at $0^{\circ}$ are threefold:

a) for $10-\mathrm{MeV}$ neutrons this reaction is the only monoenergetic source. (The neutrons from the second line have $0.033 \mathrm{MeV}$ and are, therefore, too low in energy to be a factor in this type of experiment.) 
b) for $6-\mathrm{MeV}$ neutrons the specific neutron yield is an order of magnitude larger than that of the competing $\mathrm{p}-\mathrm{T}$ and $\mathrm{d}-\mathrm{D}$ reactions (sei Fig. 1).

c) the neutrons are emitted only into a forward cone, facilitating the source shadowing and giving smaller overall room background.

Although the $t-H$ source is intrinsically monoenergetic, the background stemming from triton interactiuns with the target structure (entrance foil, beamstop) must be subtracted. Therefore, at each angle four neutron spectra must be measured ${ }^{1}$ :
a) sample in, hydrogen gas in the cell
b) sample out, hydrogen gas in the cell
c) sample in, hydrogen removed from the cell
d) sample out, hydrogen removed from the cell.

The final spectrum is obtained (after appropriate normalization) by subtracting b) and c) from the sum of a) and d). However, this procedure is not exact, because in the "gas-in" spectra the neutron yield from the beam stop is lower than in the "gas-out" case because of the energy loss of the tritons in the gas. By scattering from hydrogen it was concluded that to a first order this difference can be taken into account by downscaling the "hydrogen-out" runs by $1.015 \pm 0.005$. In the fresent experiment $s i x$ spectra were measured at each angle, because both "sample-out" runs are identical for the two samples. So, at each angle ${ }^{10} \mathrm{~B}$ and ${ }^{11} \mathrm{~B}$ were measured relative to each other. 


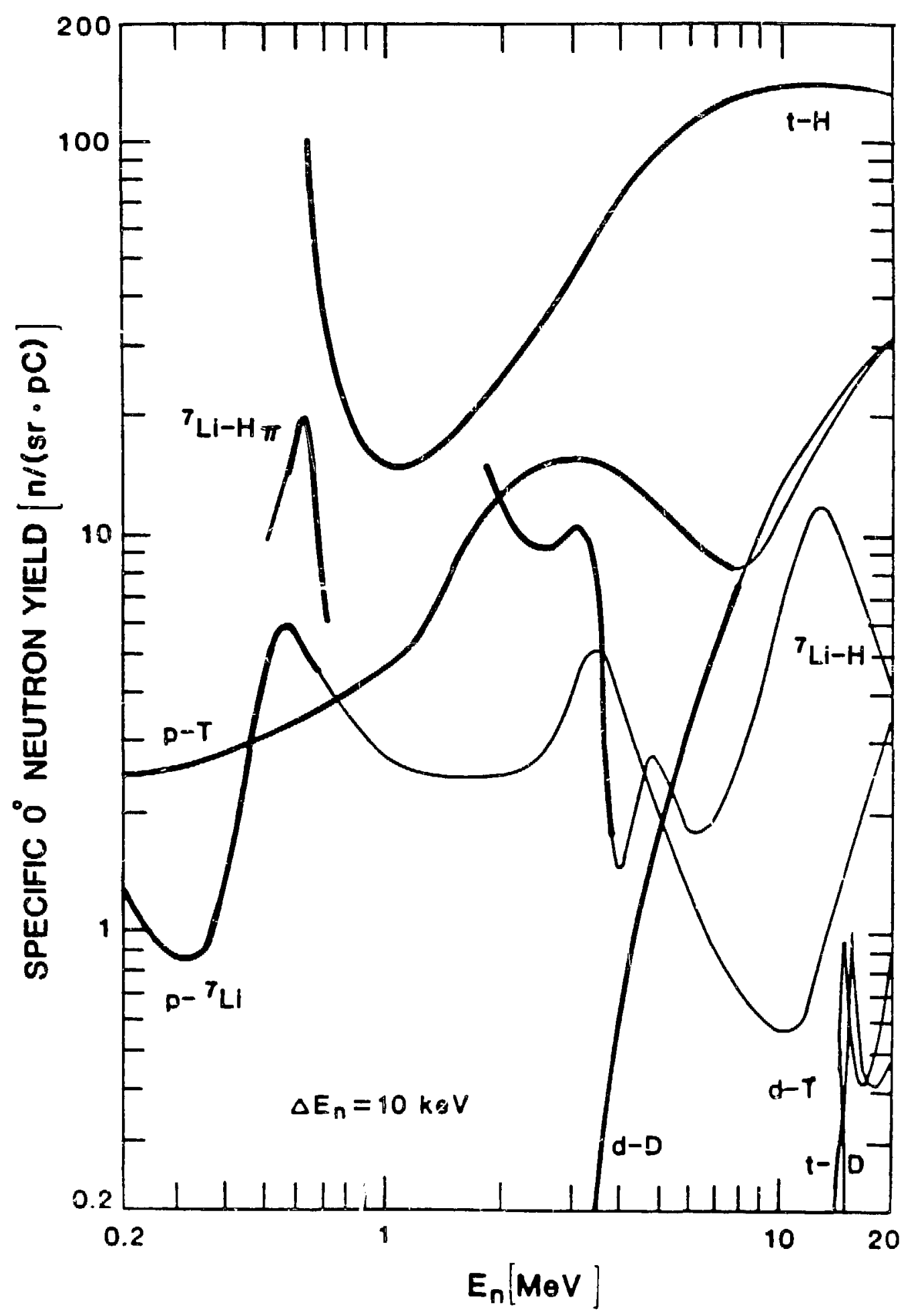

Fig. 1. Energy dependence of the specific zero-degree neutron yield. Except for $d-T$ and $t-D$ the thick curves indicate the monoenergetic range of the reaction in question. The index $\pi$ denotes the second neutron group at $0^{\circ}\left(180^{\circ}\right.$ in the C.m. systent). 
The use of a fast time digitizer ${ }^{1}$ and a rather long spacing between the neutron bursts ( $800 \mathrm{~ns}$ ) resulted ir, a dead ine of less than 18 . After dead-time correction the six spectra at each angle were normalized by using the accumulated beam charge. The "gas-in" runs were, in addition, normalized to the accumulated counts of the neutron monitor viewing the gas target. After correcting for temperature and pressure changes in the active volume of the gas target, the two methods agreed to better than 0.58 .

At $35^{\circ}$, scattering from hydrogen (using cylindrical samples of $2.92 \mathrm{~g}$ polyethelene and $2.83 \mathrm{~g}$ carbon foam with a height of $2.54 \mathrm{~cm}$ and a diameter of $1.25 \mathrm{~cm}$ as foreground and background samples respectively) was used to obtain absolute cross sections by comparison with the ${ }^{1} \mathrm{H}(\mathrm{n}, \mathrm{n}){ }^{1} \mathrm{H}$ cross section standard ${ }^{4}$.

\section{DATA REDUCTION}

The normalized net time-of-flight spectra obtained the way described in Section II were converted (in a first order relativistic approximation) to energy spectra and corrected for the energy dependence of the neutron detection efficiency. Then, at each angle, the appropriate proportion of the spectrum of the other isotope was subtracted to correct for its admixture. This way the uncorrected spectra of Tables I through IV were obtained. These spectra were then corrected for multiple scattering using the Monte Carlo simulation performed by the Los Alamos National Laboratory code $M_{C N P}{ }^{6}$ (Tables V through VIII). For these calculations the cross section library ENDF/B-IV was lised. In this simulation the scattered 
TABLE I. Uncorrected Double Diff. Neutron Emission Cross Sections for 6.0.MeV Neutrans on ${ }^{10} B$ [Data and Errors in mb/(srkMeV)]

on

\begin{tabular}{|c|c|c|c|c|c|c|c|c|c|c|c|c|c|c|c|c|c|c|c|c|}
\hline \multirow{2}{*}{$\begin{array}{l}\text { LAB.ANGLE [Deg] } \\
\text { E-RANGE [MeV] }\end{array}$} & \multicolumn{2}{|c|}{20.0} & \multicolumn{2}{|c|}{25.0} & \multicolumn{2}{|c|}{35.0} & \multicolumn{2}{|c|}{45.0} & \multicolumn{2}{|c|}{60.0} & \multicolumn{2}{|c|}{75.0} & \multicolumn{2}{|c|}{90.0} & \multicolumn{2}{|c|}{110.0} & \multicolumn{2}{|c|}{130.0} & \multicolumn{2}{|c|}{145.0} \\
\hline & 6.34 & 0.58 & 7.56 & 0.57 & 5.32 & 0.47 & 5.42 & 0.46 & 4.35 & 0.39 & 5.32 & 1.01 & 3.14 & 0.35 & 4.84 & 0.31 & 3.59 & 0.35 & 2.15 & 0.36 \\
\hline $0.50-0.65$ & 7.29 & 0.41 & 8.01 & 0.39 & 4.57 & 0.32 & 3.38 & 0.31 & 2.71 & 0.28 & 9.52 & 0.46 & 6.45 & 0.24 & 1.66 & 0.20 & 1.45 & 0.22 & 1.42 & 0.26 \\
\hline $0.65 \cdot 0.80$ & 3.27 & 0.39 & 3.68 & 0.36 & 2.62 & 0.28 & 4.51 & 0.25 & 7.40 & 0.23 & 4.91 & 0.21 & 1.17 & 0.19 & 0.76 & 0.19 & 0.91 & 0.23 & 1.20 & 0.24 \\
\hline $0.80-0.95$ & 5.51 & 0.32 & 6.95 & 0.30 & 7.46 & 0.24 & 6.73 & 0.23 & 3.47 & 0.20 & 1.38 & 0.18 & 0.81 & 0.22 & 0.65 & 0.17 & 1.10 & 0.17 & 1.09 & 0.21 \\
\hline $0.95 \cdot 1.10$ & 5.57 & 0.30 & 4.78 & 0.27 & 2.94 & 0.21 & 2.02 & 0.20 & 1.54 & 0.20 & 0.52 & 0.24 & 1.06 & 0.18 & 1.15 & 0.14 & 1.16 & 0.16 & 1.20 & 0.20 \\
\hline $1.10-1.25$ & 1.59 & 0.28 & 2.32 & 0.25 & 1.32 & 0.21 & 1.51 & 0.22 & 1.13 & 0.70 & 0.95 & 0.15 & 0.98 & 0.15 & 1.46 & 0.13 & 3.79 & 0.16 & 8.47 & 0.21 \\
\hline $1.25 \cdot 1.40$ & 1.80 & 0.30 & 2.34 & 0.28 & 1.55 & 0.24 & 1.48 & 0.20 & 1.61 & 1.18 & 1.25 & 0.14 & 1.49 & 0.14 & 5.57 & 0.15 & 10.10 & 0.18 & 4.87 & 0.19 \\
\hline $1.40-1.55$ & 1.69 & 0.26 & 2.18 & 0.23 & 1.33 & 0.18 & 1.37 & 0.17 & 1.78 & 1.24 & 1.39 & 0.14 & 4.61 & 0.15 & 11.53 & 0.17 & 1.68 & 0.14 & 1.25 & 0.17 \\
\hline $1.55 \cdot 1.70$ & 1.79 & 0.25 & 1.99 & 0.22 & 1.69 & 0.17 & 1.74 & $0.10 ́$ & 2.36 & 1.18 & 6.08 & 0.16 & 14.96 & 0.20 & 1.65 & 0.11 & 0.85 & 0.13 & 1.02 & 0.16 \\
\hline $1.70-1.85$ & 2.32 & 0.24 & 2.89 & 0.21 & 1.75 & 0.17 & 2.53 & 0.16 & 0.91 & 0.68 & 15.99 & 0.20 & 4.59 & 0.14 & 1.07 & 0.10 & 1.11 & 0.12 & 0.73 & 0.16 \\
\hline $1.85 \cdot 2.00$ & 2.27 & 0.23 & 2.58 & 0.20 & 3.37 & 0.17 & 6.84 & 0.18 & 16.37 & 0.22 & 4.50 & 0.14 & 1.10 & 0.11 & 0.89 & 0.10 & 1.03 & 0.13 & 0.74 & 0.15 \\
\hline $2.00-2.20$ & 7.18 & 0.21 & 7.87 & 0.19 & 10.92 & 0.17 & 12.81 & 0.17 & 4.41 & 0.13 & 1.02 & 0.09 & 1.05 & 0.09 & 0.98 & 0.79 & 1.11 & 0.10 & 1.19 & 0.13 \\
\hline $2.20-2.40$ & 9.48 & 0.21 & 8.92 & 0.19 & 4.98 & 0.14 & 2.90 & 0.13 & 0.91 & 0.10 & 0.80 & 0.09 & 1.00 & 0.09 & 1.01 & 0.09 & 2.38 & 0.11 & 5.59 & 0.15 \\
\hline $2.40-2.60$ & 2.59 & 0.19 & 2.46 & 0.16 & 1.91 & 0.13 & 1.25 & 0.58 & 1.00 & $0.1 C$ & 0.91 & 0.09 & 1.09 & 0.09 & 2.40 & 0.10 & 5.23 & 0.17 & 3.12 & 0.23 \\
\hline $2.60-2.80$ & 3.43 & 0.18 & 3.36 & 0.17 & 2.86 & 0.13 & 2.09 & 1.30 & 1.34 & 0.10 & 1.29 & 0.09 & 1.87 & 0.09 & 5.05 & 0.15 & 1.34 & 0.17 & 1.57 & 0.12 \\
\hline $2.80-3.00$ & 3.68 & 0.18 & 3.50 & 0.17 & 2.88 & 0.13 & 2.92 & 1.49 & 1.98 & 0.10 & 3.08 & 0.10 & 5.61 & 0.14 & 1.70 & 0.14 & 1.74 & 0.10 & 1.22 & 0.11 \\
\hline $3.00-3.20$ & 3.71 & 0.18 & 3.83 & 0.17 & 3.37 & 0.14 & 4.07 & 1.30 & 3.19 & 0.12 & 6.64 & 0.16 & 3.71 & 0.16 & 2.02 & 0.09 & 1.35 & 0.09 & 1.62 & 0.11 \\
\hline $3.20 \cdot 3.40$ & 4.52 & 0.18 & 4.05 & 0.18 & 6.32 & 0.16 & 7.65 & 0.66 & 6.75 & 0.19 & 3.61 & 0.17 & 2.33 & 0.10 & 2.15 & 0.09 & 2.41 & 0.10 & 4.01 & 0.13 \\
\hline $3.40-3.60$ & 9.11 & 0.21 & 8.23 & 0.21 & 13.70 & 0.40 & 10.51 & 0.22 & 4.15 & 0.17 & 2.17 & 0.10 & 2.11 & 0.10 & 2.67 & 0.10 & 6.36 & 0.13 & 11.28 & 0.17 \\
\hline $3.60 \cdot 3.80$ & 13.14 & 0.28 & 12.31 & 0.29 & 13.81 & 0.97 & 4.39 & 0.18 & 2.34 & 0.11 & 1.95 & 0.09 & 2.22 & 0.10 & 4.86 & 0.12 & 11.58 & 0.17 & 10.58 & 0.17 \\
\hline $3.80 \cdot 4.00$ & 7.91 & 0.23 & 7.09 & 0.24 & 4.46 & 1.78 & 3.28 & 0.12 & 2.06 & 0.10 & 1.91 & 0.10 & 3.03 & 0.11 & 9.70 & 0.15 & 12.16 & 0.18 & 30.09 & 0.28 \\
\hline $4.00 \cdot 4.25$ & 6.33 & 0.18 & 7.22 & 0.20 & 4.98 & 1.41 & 3.05 & 0.11 & 1.91 & 0.10 & 2.88 & 0.10 & 6.18 & 0.12 & 11.87 & 0.16 & 46.06 & 0.36 & 79.78 & 0.65 \\
\hline $4.25-4.50$ & 6.63 & 0.19 & 6.90 & 0.58 & 2.40 & 0.69 & 2.89 & 0.11 & 2.80 & 0.10 & 6.12 & 0.12 & 9.15 & 0.14 & 44.06 & 0.35 & 59.33 & 0.85 & 25.05 & 0.84 \\
\hline $4.50-4.75$ & 11.45 & 0.24 & 13.08 & 1.08 & 2.45 & 0.36 & 4.88 & 0.12 & 6.01 & 0.13 & 8.36 & 0.14 & 22.21 & 0.7 .0 & 71.51 & 0.79 & 13.10 & 0.48 & 7.71 & 0.17 \\
\hline $4.75 \cdot 5.00$ & 21.53 & 0.73 & 11.10 & 2.17 & 12.44 & 0.17 & 11.34 & 0.17 & 9.28 & 0.15 & 19.40 & 0.22 & 61.00 & 0.59 & 22.08 & 0.51 & 11.00 & 0.16 & 7.78 & 0.13 \\
\hline $5.00 \cdot 5.25$ & 45.79 & $1.5 i$ & 31.83 & 1.10 & 32.03 & 0.29 & 25.04 & 0.26 & 19.69 & 0.25 & 43.24 & 0.40 & 43.51 & 0.53 & 12.37 & 0.16 & 3.48 & 0.10 & & \\
\hline $5.25 \cdot 5.50$ & 99.35 & 2.46 & 80.77 & 0.83 & 90.32 & 0.68 & 68.62 & 0.55 & 45.69 & 0.46 & 28.96 & 0.38 & 14.49 & 0.19 & 2.79 & 0.08 & 0.40 & 0.06 & & \\
\hline $5.50-5.73$ & 259.60 & 2.27 & 209.25 & 1.51 & 207.06 & 1.52 & 133.71 & 1.08 & 38.44 & 0.40 & 8.33 & 0.15 & 4.15 & 0.10 & 0.34 & 0.05 & 0.15 & 0.05 & & \\
\hline $5.75 \cdot 6.00$ & 406.55 & 3.24 & 363.19 & 2.90 & 181.65 & 1.73 & 74.96 & 0.87 & 7.84 & 0.14 & 1.31 & 0.07 & 0.37 & 0.06 & 0.05 & 0.04 & & & & \\
\hline $6.00-6.25$ & 188.56 & 1.82 & 167.44 & 1.69 & 27.91 & 0.44 & 6.28 & 0.16 & 0.83 & 0.06 & 0.19 & 0.05 & 0.20 & 0.05 & & & & & & \\
\hline $6.25-6.50$ & 17.14 & 0.27 & 10.06 & 0.19 & 1.76 & 0.08 & 0.49 & 0.06 & 0.05 & 0.05 & & & & & & & & & & \\
\hline $6.50-6.75$ & 1.98 & 0.10 & 1.46 & 0.09 & 0.70 & 0.06 & 0.10 & 0.06 & & & & & & & & & & & & \\
\hline $6.75 \cdot 7.00$ & 0.88 & 0.09 & 0.72 & 0.08 & 0.24 & 0.06 & & & & & & & & & & & & & & \\
\hline
\end{tabular}




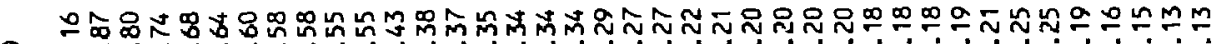

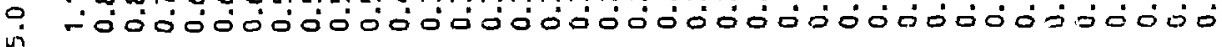

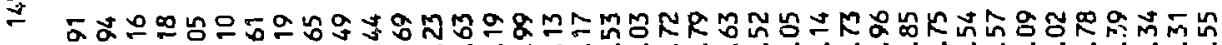

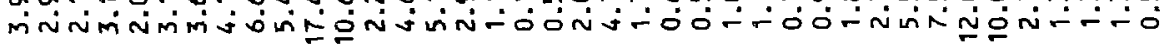

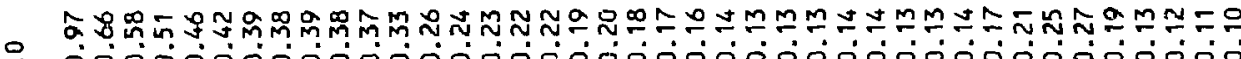

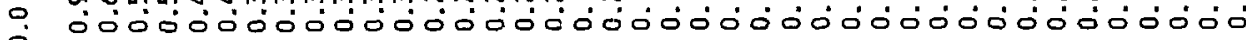

$\stackrel{\rho}{m}$

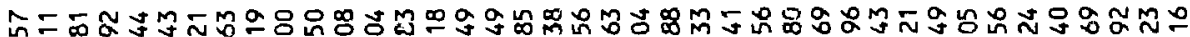

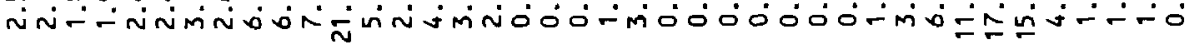

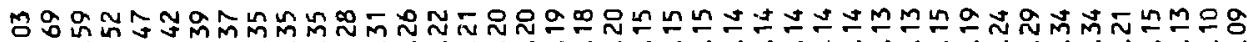

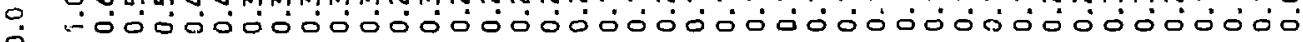

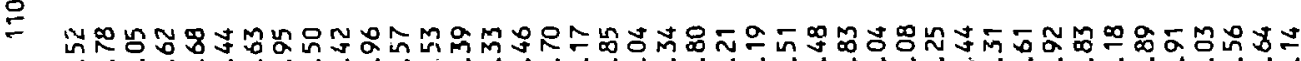

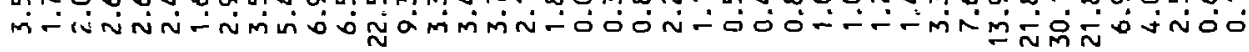

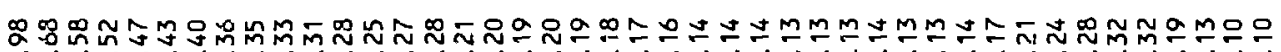

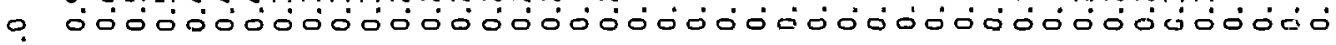

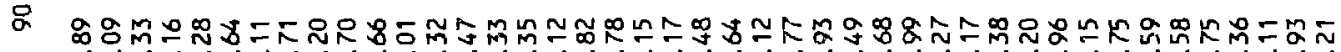

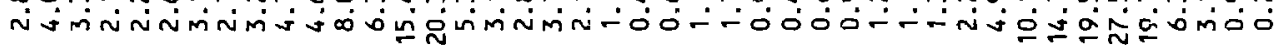

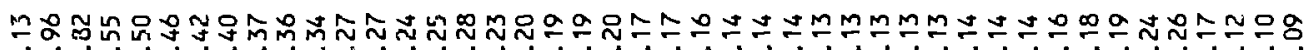

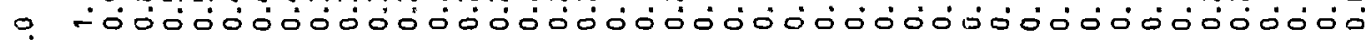

는 n-

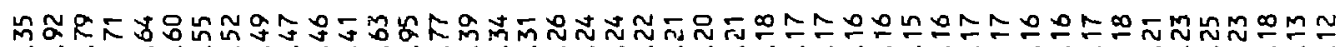

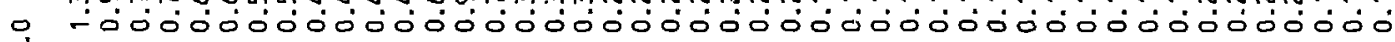

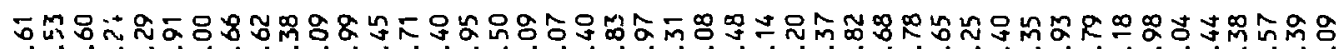

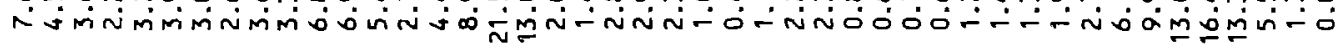

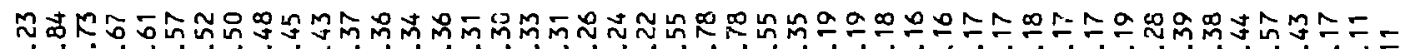

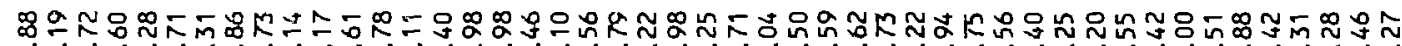

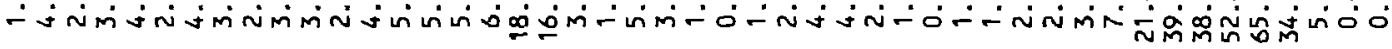

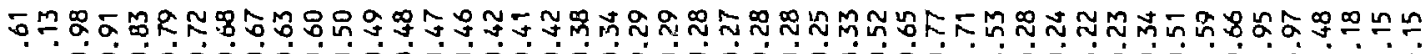

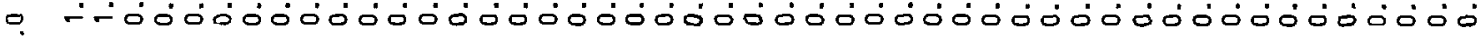

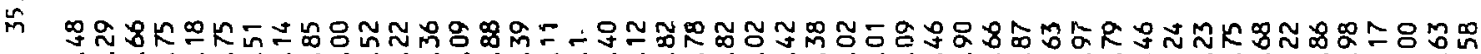

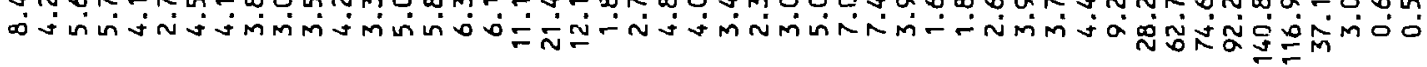

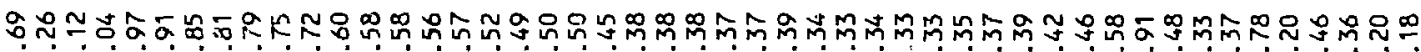

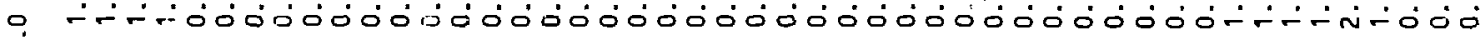

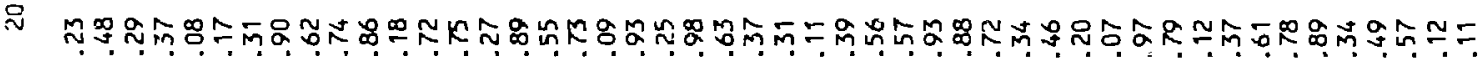
nиं $\stackrel{g}{0}$

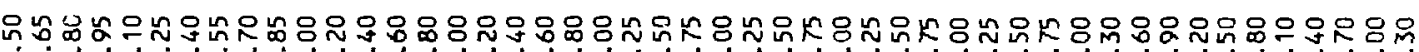
ú

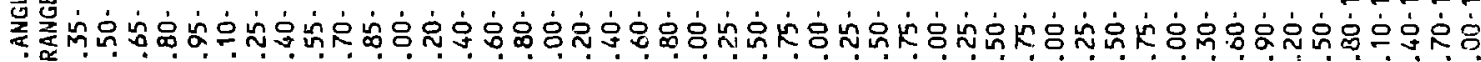

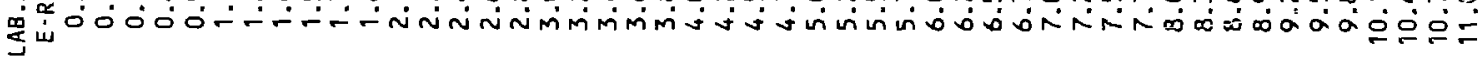


TABLE 111. Uncorrected Louble Diff. Neutron Emission Cross Sections for 6.0-MeV Neutrons on $11^{B}$ [Data and Errors in mb/(srkMeV)]

\begin{tabular}{|c|c|c|c|c|c|c|c|c|c|c|c|c|c|c|c|c|c|c|c|c|}
\hline $\begin{array}{l}\text { LAB.ANGLE [Deg] } \\
\text { E-RANGE [MeV] }\end{array}$ & \multicolumn{2}{|c|}{20.0} & \multicolumn{2}{|c|}{25.0} & \multicolumn{2}{|c|}{35.0} & \multicolumn{2}{|c|}{45.0} & \multicolumn{2}{|c|}{60.0} & \multicolumn{2}{|c|}{75.0} & \multicolumn{2}{|c|}{90.0} & \multicolumn{2}{|c|}{110.0} & \multicolumn{2}{|c|}{130.0} & \multicolumn{2}{|c|}{145.0} \\
\hline $0.35 \cdot 0.50$ & 6.05 & 1.11 & 7.83 & 1.08 & 5.32 & 0.89 & 6.57 & 0.85 & 8.80 & 0.73 & 16.25 & 0.68 & 14.33 & 0.68 & 3.11 & 0.60 & 2.31 & 0.65 & 2.41 & 0.66 \\
\hline $0.50-0.65$ & 14.51 & 0.77 & 18.10 & 0.72 & 21.40 & 0.59 & 26.93 & 0.58 & 22.39 & 0.51 & 8.78 & 0.44 & 3.48 & 0.42 & 2.13 & 0.38 & 6.12 & 0.42 & 11.36 & 0.50 \\
\hline $0.65-0.80$ & 38.44 & 0.71 & 34.95 & 0.66 & 24.23 & 0.52 & 11.37 & 0.46 & 2.00 & 0.39 & 2.35 & 0.35 & 3.33 & $0.3 j$ & 18.64 & 0.39 & 24.84 & 0.44 & 13.36 & 0.47 \\
\hline $0.80-0.95$ & 5.78 & 0.60 & 5.76 & 0.53 & 2.22 & 0.42 & 2.29 & 0.38 & 3.12 & 0.34 & 7.14 & 0.33 & 28.38 & 0.43 & 17.91 & 0.38 & 3.40 & 0.35 & 3.20 & 0.44 \\
\hline $0.95-1.10$ & 2.44 & 0.56 & 3.74 & 0.50 & 3.64 & 0.39 & 4.37 & 0.36 & 13.21 & 0.37 & 37.01 & 0.44 & 15.82 & 0.37 & 1.71 & 0.29 & 2.50 & 0.31 & 0.49 & 0.37 \\
\hline $1.10-1.25$ & 6.61 & 0.55 & 8.49 & 0.49 & 11.58 & 0.41 & 23.73 & 0.43 & 33.74 & 0.44 & 7.54 & 0.30 & 0.88 & 0.28 & 0.27 & 0.25 & 0.46 & 0.27 & 0.49 & 0.37 \\
\hline $1.25-1.40$ & 30.85 & 0.57 & 31.49 & 0.53 & 30.67 & 0.44 & 20.55 & 0.40 & 3.02 & 0.31 & 0.45 & 0.25 & 0.47 & 0.27 & 0.27 & 0.25 & 0.36 & 0.26 & 0.17 & 0.33 \\
\hline $1.40-1.55$ & 14.98 & 0.51 & 11.91 & 0.45 & 3.24 & 0.33 & 1.83 & 0.29 & 1.88 & 0.30 & 0.99 & 0.24 & 0.25 & 0.25 & 0.24 & 0.25 & 0.36 & 0.26 & 0.40 & 0.31 \\
\hline $1.55 \cdot 1.70$ & 2.01 & 0.46 & 2.43 & 0.40 & 1.36 & 0.31 & 1.36 & 0.29 & 1.14 & 0.29 & 0.83 & 0.24 & 0.27 & 0.26 & 1.04 & 0.21 & 0.54 & 0.24 & $1.2^{7}$ & 0.29 \\
\hline $1.70-1.85$ & 3.20 & 0.44 & 3.19 & 0.38 & 2.49 & 0.30 & 2.12 & 0.27 & 1.20 & 0.26 & 0.28 & 0.28 & 0.27 & 0.26 & 1.32 & 0.21 & 1.64 & 0.23 & 1.02 & 0.29 \\
\hline $1.85-2.00$ & 2.20 & 0.43 & 3.66 & 0.36 & 2.13 & 0.29 & 1.09 & 0.27 & 1.05 & 0.29 & 1.71 & 0.22 & 1.97 & 0.22 & 1.67 & 0.20 & 2.01 & 0.23 & 1.80 & 0.28 \\
\hline $2.00-2.20$ & 2.27 & 0.37 & 1.74 & 0.32 & 1.24 & 0.26 & 1.29 & 0.26 & 1.66 & 0.21 & 1.89 & 0.18 & 2.26 & 0.19 & 2.30 & 0.18 & 2.77 & 0.20 & 2.34 & 0.24 \\
\hline $2.20-2.40$ & 2.39 & 0.37 & 2.92 & 0.32 & 2.04 & 0.24 & 1.68 & 0.22 & 1.33 & 0.19 & 2.02 & 0.17 & 2.13 & 0.18 & 2.96 & 0.18 & 4.33 & 0.20 & 7.46 & 0.27 \\
\hline $2.40-2.60$ & 3.11 & 0.34 & 3.38 & 0.29 & 2.83 & 0.23 & 1.81 & 0.22 & 1.45 & 0.18 & 2.07 & 0.16 & 2.49 & 0.18 & 3.73 & 0.18 & 21.16 & 0.30 & 37.67 & 0.39 \\
\hline $2.60 \cdot 2.80$ & 3.78 & 0.33 & 3.84 & 0.28 & 3.76 & 0.22 & 2.53 & 0.25 & 1.72 & 0.18 & 2.06 & 0.17 & 3.26 & 0.18 & 20.36 & 0.29 & 27.35 & 0.32 & 7.26 & 0.25 \\
\hline $2.80 \cdot 3.00$ & & 0.33 & 6.03 & 0.29 & 4.89 & 0.23 & 4.29 & 0.27 & & 0.18 & & 0.18 & 14.57 & 0.28 & 22.60 & 0.29 & 3.00 & 0.18 & 1.76 & 0.21 \\
\hline $3.00 \cdot 3.20$ & 7.18 & 0.33 & 7.51 & 0.31 & 6.43 & 0.25 & 6.28 & 0.27 & 7.89 & 0.22 & 17.92 & 0.29 & 23.45 & 0.32 & 2.87 & 0.16 & & 0.17 & 1.79 & 0.21 \\
\hline $3.20 \cdot 3.40$ & 8.20 & 0.33 & 7.51 & 0.32 & 7.95 & 0.27 & 9.51 & 0.28 & 24.44 & 0.33 & 25.74 & 0.32 & 6.59 & 0.21 & 3.10 & 0.16 & 2.14 & 0.18 & 1.57 & 0.22 \\
\hline $3.40-3.60$ & 15.70 & 0.38 & 15.14 & 0.38 & 22.64 & 0.38 & 29.56 & 0.39 & 23.05 & 0.32 & & 0.20 & $4.1 i$ & 0.19 & 4.53 & 0.18 & 3.31 & 0.21 & 2.30 & 0.25 \\
\hline $3.60-3.80$ & 36.20 & 0.47 & 36.92 & 0.50 & 33.47 & 0.43 & 23.50 & 0.34 & 3.88 & 0.20 & 3.00 & 0.17 & 3.64 & 0.19 & 4.66 & 0.19 & & 0.25 & 5.38 & 0.28 \\
\hline $3.80-4.00$ & 23.39 & 0.42 & 22.46 & 0.45 & 9.66 & 0.35 & 4.22 & 0.22 & 2.55 & 0.19 & 2.38 & 0.17 & 3.74 & 0.19 & 5.27 & 0.22 & 9.67 & 0.29 & 20.37 & 0.48 \\
\hline $4.00-4.25$ & 7.21 & 0.32 & 6.08 & 0.35 & 4.42 & 0.28 & 3.14 & 0.19 & 2.40 & 0.18 & 2.87 & 0.16 & 4.80 & 0.20 & 9.87 & 0.25 & 44.17 & 0.65 & 111.96 & 1.09 \\
\hline $4.25 \cdot 4.50$ & 6.36 & 0.35 & 6.69 & 0.35 & 4.54 & 0.23 & 3.71 & 0.19 & 2.96 & 0.19 & 3.74 & 0.19 & 8.03 & 0.24 & 41.93 & 0.63 & 160.21 & 1.01 & 166.94 & 0.81 \\
\hline $4.50-4.75$ & 6.47 & 0.44 & 6.68 & 0.37 & 5.32 & 0.22 & 3.69 & 0.20 & 3.96 & 0.20 & 6.61 & 0.23 & 29.85 & 0.44 & 141.58 & 1.07 & 91.91 & 0.58 & 20.34 & 0.31 \\
\hline $4.75-5.00$ & 11.92 & 0.63 & 10.97 & 0.42 & 8.62 & 0.29 & 6.51 & 0.26 & 8.27 & 0.25 & 19.43 & 0.37 & 97.28 & 0.93 & 96.11 & 0.63 & 7.91 & 0.25 & 3.07 & 0.20 \\
\hline $5.00-5.25$ & 35.28 & 0.99 & 26.29 & 0.55 & 30.37 & 0.52 & 23.30 & 0.44 & 27.35 & 0.41 & 54.42 & 0.67 & 89.49 & 0.78 & 10.12 & 0.26 & 1.34 & 0.14 & 0.61 & 0.12 \\
\hline $5.25 \cdot 5.50$ & 130.43 & 1.71 & 98.34 & 1.12 & 108.71 & 1.18 & 78.54 & 0.95 & 69.59 & 6.73 & 57.88 & 0.57 & 12.32 & 0.30 & 1.38 & 0.13 & 0.28 & 0.10 & & \\
\hline
\end{tabular}






年 mí-

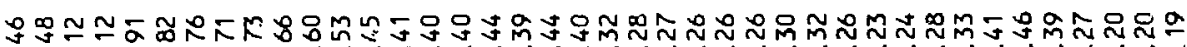
$\because-\therefore$ -

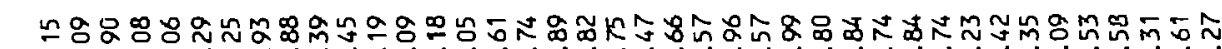
0ं0ல0்

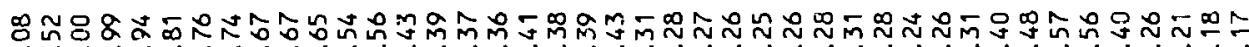
i- -

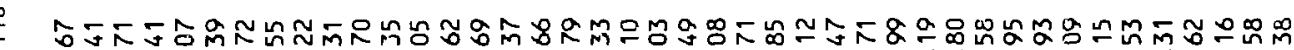
ப-

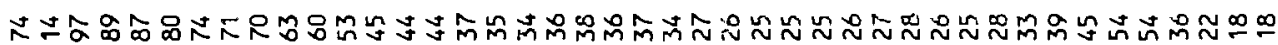

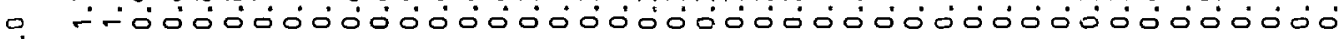

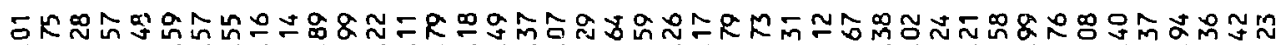

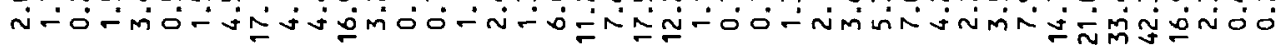





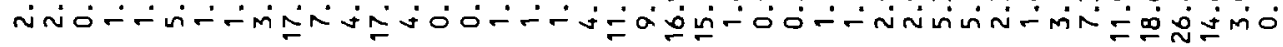

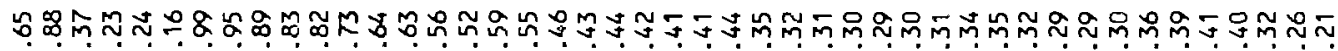
N亡- - -

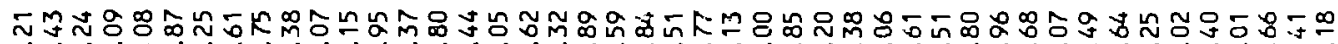

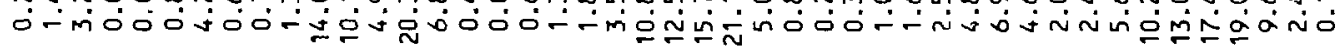

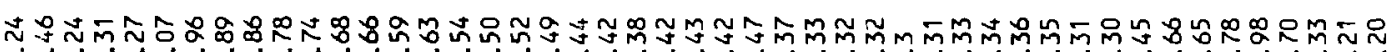

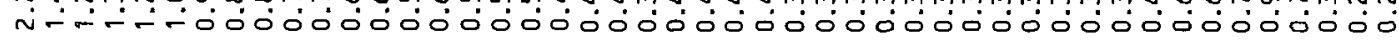

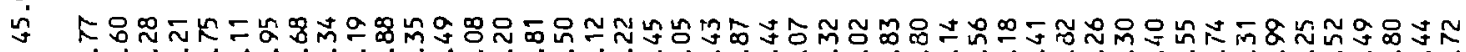


бํํำ

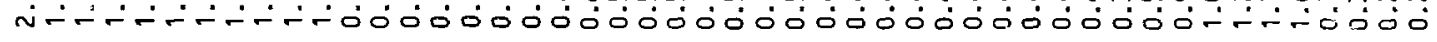

卢

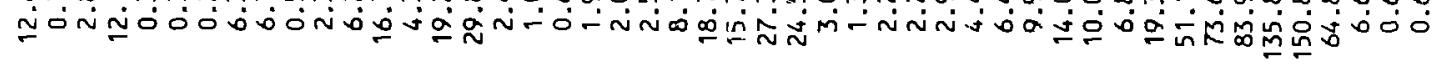

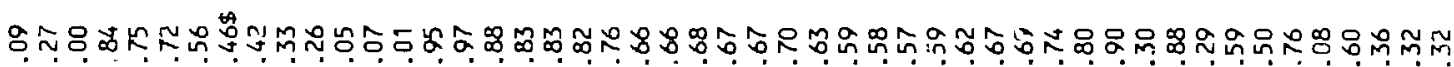

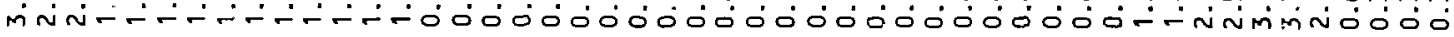

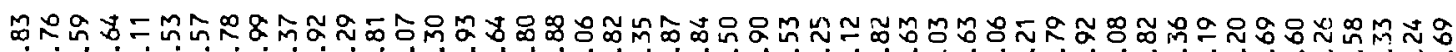
نं

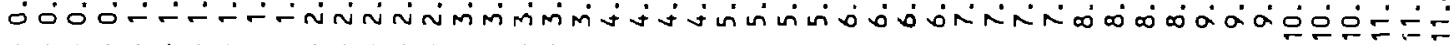




\begin{tabular}{|c|c|c|c|c|c|c|c|c|c|c|c|c|c|c|c|c|c|c|c|c|}
\hline \multirow{2}{*}{$\begin{array}{l}\text { LAB.ANGLE [Deg] } \\
\text { E-RANGE [MeV] }\end{array}$} & \multicolumn{2}{|c|}{20.0} & \multicolumn{2}{|c|}{25.0} & \multicolumn{2}{|c|}{35.0} & \multicolumn{2}{|c|}{45.0} & \multicolumn{2}{|c|}{60.0} & \multicolumn{2}{|c|}{75.0} & \multicolumn{2}{|c|}{90.0} & \multicolumn{2}{|c|}{110.0} & \multicolumn{2}{|c|}{130.0} & \multicolumn{2}{|c|}{145.0} \\
\hline & & & & & & & & & & & & & & & & & & & & \\
\hline $0.35-0.50$ & 8.7 & 1.5 & 10.6 & 2.6 & 8.6 & 2.1 & 7.9 & 1.4 & 5.9 & 0.9 & 6.3 & 1.2 & 2.5 & 0.4 & 6.1 & 0.8 & 4.3 & 0.6 & 2.8 & 0.5 \\
\hline $0.50-0.65$ & 7.5 & 0.5 & 8.4 & 0.6 & 3.8 & 0.5 & 7.1 & 2.0 & 5.6 & 1.6 & 12.1 & 1.5 & 8.1 & 0.9 & 1.2 & 0.3 & 1.0 & 0.3 & 1.0 & 0.3 \\
\hline $0.65 \cdot 0.80$ & 3.2 & 0.4 & 3.7 & 0.4 & 1.3 & 0.7 & 8.4 & 2.1 & 10.5 & 1.6 & 7.7 & 1.5 & 3.2 & 1.1 & 0.1 & 0.4 & 0.3 & 0.3 & 0.6 & 0.3 \\
\hline $0.80 \cdot 0.95$ & 9.6 & 2.3 & 11.6 & 3.8 & 11.3 & 2.3 & 7.2 & 0.4 & 3.8 & 0.3 & 1.7 & 0.3 & 0.9 & 0.2 & 0.5 & 0.2 & 0.9 & 0.2 & 0.9 & 0.2 \\
\hline $0.95-1.10$ & 11.0 & 3.0 & 10.9 & 4.9 & 8.3 & 3.3 & 1.4 & 0.3 & 1.0 & 0.3 & 0.04 & 0.3 & 0.6 & 0.3 & 1.2 & 0.2 & 1.1 & 0.2 & 1.1 & 0.2 \\
\hline $1.10-1.25$ & 3.2 & 1.0 & 4.3 & 1.6 & 2.9 & 1.0 & 1.1 & 0.3 & 0.7 & 0.7 & 0.9 & 0.2 & 0.9 & 0.1 & 2.1 & 0.4 & 4.2 & 0.3 & 8.9 & 0.4 \\
\hline $1.25-1.40$ & 1.4 & 0.4 & 2.1 & 0.3 & 1.1 & 0.3 & 1.1 & 0.3 & 1.2 & 1.2 & 1.5 & 0.2 & 1.6 & 0.2 & 6.5 & 0.6 & 10.8 & 0.5 & 5.6 & 0.5 \\
\hline $1.40 \cdot 1.55$ & 1.1 & 0.4 & 1.7 & 0.4 & 0.7 & 0.4 & $1 . \overline{3}$ & 0.2 & 1.7 & 1.2 & 2.2 & 0.5 & 5.2 & 0.4 & 12.0 & 0.3 & 2.0 & 0.3 & 1.6 & 0.3 \\
\hline $1.55 \cdot 1.70$ & 1.1 & 0.4 & 1.3 & 0.5 & 0.9 & 0.4 & 2.0 & 0.2 & 2.5 & 1.2 & 7.4 & 0.8 & 16.0 & 0.7 & 1.6 & 0.1 & 0.8 & 0.1 & $1 . n$ & 0.2 \\
\hline $1.70-1.85$ & 2.0 & 0.3 & 2.6 & 0.2 & 1.4 & 0.2 & 3.4 & 0.6 & 7.6 & 0.8 & 16.8 & 0.5 & 5.2 & 0.4 & 0.9 & 0.1 & 0.9 & 0.1 & 0.7 & 0.2 \\
\hline $1.85 \cdot 2.00$ & 2.5 & 0.3 & 3.0 & 0.5 & 3.5 & 0.3 & 8.7 & 1.1 & 17.9 & 0.9 & 4.6 & 0.2 & 1.1 & 0.1 & 0.4 & 0.2 & 0.6 & 0.2 & 0.7 & 0.2 \\
\hline $2.00-2.20$ & 8.5 & 0.8 & 9.4 & 1.4 & 12.2 & 0.9 & 14.0 & 0.7 & 5.4 & 0.6 & 0.8 & 0.1 & 0.3 & 0.1 & 0.7 & 0.9 & 0.8 & 0.1 & 1.5 & 0.3 \\
\hline $2.20-2.40$ & 12.0 & 1.4 & 11.8 & 2.4 & 7.5 & 1.6 & 3.3 & 0.3 & 9.2 & 0.2 & 0.4 & 0.2 & 0.5 & 0.2 & 1.0 & 0.1 & 2.1 & 0.1 & 6.2 & 0.6 \\
\hline $2.40-2.60$ & 3.3 & 0.6 & 3.5 & 1.0 & 2.7 & 0.6 & 0.9 & 0.6 & 0.6 & 0.1 & 0.4 & 0.2 & 1.1 & 0.1 & 3.4 & 0.6 & 5.9 & 0.5 & 3.3 & 0.3 \\
\hline $2.60-2.80$ & 3.6 & 0.3 & 3.7 & 0.5 & 3.0 & 0.3 & 1.5 & 1.3 & 1.0 & 0.1 & 0.8 & 0.1 & 2.3 & 0.4 & 5.9 & 0.6 & 2.0 & 0.5 & 1.6 & 0.2 \\
\hline $2.80 \cdot 3.00$ & 2.9 & 0.3 & 2.7 & 0.3 & 2.0 & 0.3 & 2.1 & 1.5 & 1.8 & 0.2 & 2.8 & 0.2 & 6.8 & 0.9 & 1.9 & 3.3 & 2.0 & 0.3 & 0.9 & 0.1 \\
\hline $3.00 \cdot 3.20$ & 2.6 & 0.3 & 2.9 & 0.3 & 3.0 & 0.2 & 3.6 & 1.3 & 3.8 & 0.7 & 7.3 & 0.7 & 4.0 & 0.6 & 1.5 & 0.2 & 1.2 & 0.2 & 1.2 & 0.2 \\
\hline $3.20-3.40$ & 3.4 & 0.3 & 3.3 & 0.2 & 6.3 & 0.5 & 7.5 & 0.7 & 7.6 & 0.8 & 4.5 & 0.9 & 2.2 & 0.4 & 1.3 & 0.2 & 2.0 & 0.2 & 3.5 & 0.2 \\
\hline $3.40-3.60$ & 8.7 & 0.4 & 8.8 & 1.1 & 15.4 & 1.6 & 12.0 & 1.2 & 4.0 & 0.4 & 2.3 & 0.5 & 1.1 & 0.2 & 1.7 & 0.3 & 5.3 & 0.5 & 9.8 & 0.6 \\
\hline $3.60 \cdot 3.80$ & 14.1 & 1.0 & 13.8 & 1.8 & 14.9 & 9.4 & 5.4 & 0.9 & 2.0 & 0.3 & 1.5 & 0.3 & 1.3 & 0.2 & 3.9 & 0.5 & 12.7 & 0.7 & 10.4 & 0.3 \\
\hline $3.80 \cdot 4.00$ & 10.1 & $i .6$ & 9.4 & 2.4 & 5.0 & 1.9 & 3.0 & 0.6 & 1.6 & 0.2 & 1.0 & 0.2 & 2.1 & 0.3 & 8.8 & 0.6 & 15.2 & 0.6 & 31.0 & 0.3 \\
\hline $4.00-4.25$ & 7.5 & 0.9 & 8.2 & 1.2 & 4.9 & 1.4 & 2.5 & 0.2 & 1.1 & 0.2 & 1.9 & 0.3 & 5.4 & 0.7 & 16.3 & 0.9 & 59.2 & 2.7 & 90.9 & 2.3 \\
\hline $4.25 \cdot 4.50$ & 6.7 & 0.4 & 7.0 & 0.7 & 1.9 & 0.7 & 2.6 & 0.3 & 2.0 & 0.3 & 5.3 & 0.7 & 12.8 & 0.7 & 58.0 & 2.8 & 64.8 & 1.4 & 30.9 & 1.4 \\
\hline $4.50-4.73$ & 11.3 & 0.3 & 12.9 & 1.1 & 2.1 & 0.4 & 4.9 & 0.5 & 5.4 & 0.5 & 9.4 & 0.5 & 31.3 & 1.8 & 84.3 & 2.7 & 13.6 & 0.5 & 5.3 & 0.4 \\
\hline $4 . \pi 5 \cdot 5.00$ & 21.5 & 0.9 & 11.8 & 2.3 & 12.3 & 0.6 & 12.2 & 0.7 & 9.5 & 0.6 & 23.7 & 0.8 & 73.8 & 2.6 & 26.2 & 1.0 & 9.9 & 0.3 & 7.4 & 0.2 \\
\hline $5.00-5.25$ & 45.4 & 1.8 & 36.0 & 1.9 & 31.7 & 1.1 & 28.7 & 0.7 & 23.4 & 0.7 & 51.6 & 1.7 & 49.8 & 1.4 & 91.5 & 0.2 & 3.4 & 0.1 & & \\
\hline $5.25-5.50$ & 145.1 & 9.3 & 120.0 & 7.7 & 112.0 & 4.2 & 83.8 & 3.1 & 53.2 & 1.6 & 33.0 & 0.9 & 94.7 & 0.2 & 2.8 & 0.1 & 0.4 & 0.1 & & \\
\hline $5.50-5.75$ & 345.3 & 17.2 & 275.3 & 13.2 & 248.4 & 8.3 & 154.0 & 4.2 & 43.9 & 1.2 & 9.2 & 0.2 & 3.8 & 0.7 & 0.3 & 0.05 & 0.1 & 0.05 & & \\
\hline $5.75 \cdot 6.00$ & 496.7 & 18.3 & 431.4 & 13.9 & 225.5 & 8.9 & 93.2 & 3.7 & 10.2 & 0.5 & 0.8 & 0.1 & 0.3 & 0.1 & 0.1 & 0.04 & & & & \\
\hline $6.00-6.25$ & 239.7 & 10.4 & 208.0 & 8.3 & 53.1 & 5.1 & 15.0 & 1.8 & 0.5 & 0.1 & 0.2 & 0.05 & 0.2 & 0.05 & & & & & & \\
\hline $6.25-6.50$ & 17.0 & 0.3 & 13.0 & 0.6 & 1.8 & 0.1 & 0.5 & 0.1 & 0.05 & 0.05 & & & & & & & & & & \\
\hline $6.50 \cdot 6.75$ & 1.9 & 0.1 & 2.1 & 0.2 & 0.7 & 0.1 & $0 . ?$ & 0.1 & & & & & & & & & & & & \\
\hline $6.75 \cdot 7.00$ & 0.9 & 0.1 & 0.7 & 0.1 & 0.2 & 0.1 & & & & & & & & & & & & & & \\
\hline
\end{tabular}







TABLE VII. Corrected Double Diff. Neutron Emission Cross Sections for 6.0-MeV Neutrons on ${ }^{11} B$ [Data and Errors in mb/(sr^MeV)]

\begin{tabular}{|c|c|c|c|c|c|c|c|c|c|c|c|c|c|c|c|c|c|c|c|c|}
\hline \multicolumn{2}{|c|}{$\begin{array}{l}\text { LAB.ANGLE [Deg] } \\
\text { E-RANGE [MeV] }\end{array}$} & 20.0 & \multicolumn{2}{|c|}{25.0} & \multicolumn{2}{|c|}{35.0} & \multicolumn{2}{|c|}{45.0} & \multicolumn{2}{|c|}{60.0} & \multicolumn{2}{|c|}{75.0} & \multicolumn{2}{|c|}{90.0} & \multicolumn{2}{|c|}{$110 . C$} & \multicolumn{2}{|c|}{130.0} & \multicolumn{2}{|c|}{145.0} \\
\hline $0.35 \cap .50$ & 4.2 & 1.9 & 6.1 & 1.9 & 3.7 & 1.6 & 7.6 & 1.4 & 9.7 & 1.4 & 17.3 & 1.2 & 13.1 & 0.9 & 3.1 & 0.6 & 2.0 & 0.7 & 1.7 & 0.7 \\
\hline $0.50-0.65$ & 13.8 & 0.9 & 17.4 & 0.9 & 20.7 & 0.8 & 27.9 & 1.1 & 23.2 & 1.1 & 10.5 & 1.7 & 1.9 & 1.0 & 3.1 & 0.8 & 6.8 & 0.8 & 11.7 & 0.6 \\
\hline $0.65-0.80$ & 40.2 & 1.9 & 36.3 & 1.7 & 25.6 & 1.4 & 9.6 & 1.5 & 0.4 & 1.8 & 3.0 & 0.3 & 3.6 & 0.5 & 22.4 & 2.3 & 28.2 & 2.5 & 16.1 & 1.7 \\
\hline $0.80-0.95$ & 4.6 & 1.1 & 4.9 & 0.8 & 1.1 & 0.9 & 3.3 & 1.1 & 3.9 & 1.2 & 12.4 & 5.1 & 33.1 & 3.1 & 17.7 & 0.4 & 2.9 & 0.5 & 2.2 & 0.6 \\
\hline $0.95 \cdot 1.10$ & 4.1 & 1.9 & 5.8 & 2.4 & 4.8 & 1.4 & 9.8 & 5.0 & 17.8 & 5.6 & 39.3 & 2.3 & 17.9 & 1.4 & 0.2 & 0.5 & 1.5 & 0.6́ & 0.3 & 0.5 \\
\hline $1.10-1.25$ & 13.7 & 6.9 & 15.5 & 7.4 & 17.3 & 5.3 & 29.7 & 5.5 & 38.7 & 5.9 & 7.9 & 0.5 & 1.3 & 0.4 & 0.2 & 0.5 & 0.1 & 0.5 & 0.1 & 0.4 \\
\hline $1.25-1.40$ & 46.0 & 14.3 & 45.5 & 14.4 & 43.1 & 11.2 & 23.4 & 2.7 & 4.9 & $2 . \dot{2}$ & 0.1 & 0.6 & $0 . i$ & 0.3 & 0.0 & 0.3 & 0.3 & 0.3 & 0.2 & 0.3 \\
\hline $1.40-1.55$ & 20.1 & 4.9 & 17.6 & 5.9 & 7.5 & 3.8 & 1.3 & 0.3 & 1.6 & 0.4 & 0.7 & 0.2 & 0.2 & 0.3 & U.2 & 0.3 & 0.3 & 0.3 & 0.4 & 0.3 \\
\hline $1.55-1.70$ & 3.4 & 1.5 & 4.3 & 2.1 & 2.6 & 1.2 & 1.2 & 0.3 & 0.9 & 0.3 & 0.7 & 0.2 & 0.2 & 0.3 & 1.0 & 0.2 & 0.5 & 0.2 & 1.2 & 0.3 \\
\hline $1.70 \cdot 1.85$ & 2.8 & 0.6 & 3.0 & 0.4 & 2.1 & 0.4 & 1.8 & 0.4 & 0.9 & 0.4 & 0.0 & 0.3 & 0.1 & 0.3 & 1.2 & 0.2 & 1.5 & 0.2 & 0.9 & 0.3 \\
\hline $1.85-2.00$ & 1.7 & 0.6 & 3.1 & 0.6 & 1.7 & 0.5 & 0.7 & 0.4 & 0.7 & 0.5 & 1.3 & 0.4 & 1.6 & 0.3 & 1.4 & 0.2 & 1.8 & 0.2 & 1.6 & 0.3 \\
\hline $2.00-2.20$ & 1.7 & 0.6 & 1.2 & 0.6 & 0.7 & 0.5 & 0.8 & 0.5 & 1.2 & 0.5 & 1.4 & 0.5 & 1.7 & 0.3 & 1.7 & 0.3 & 2.1 & 0.4 & 1.8 & 0.3 \\
\hline $2.20-2.40$ & 1.9 & 0.6 & 2.4 & 0.6 & 1.6 & 0.4 & 1.2 & 0.4 & 0.9 & 0.5 & 1.5 & 0.4 & 1.5 & 0.4 & 2.2 & 0.4 & 4.8 & 0.6 & 7.5 & 0.4 \\
\hline $2.40-2.60$ & 2.7 & 0.5 & 2.9 & 0.5 & 1.8 & 0.4 & 1.4 & 0.4 & 1.0 & 0.5 & 1.6 & 0.4 & 1.8 & 0.4 & 4.2 & 0.4 & 24.8 & 2.8 & 40.3 & 1.8 \\
\hline $2.60 \cdot 2.80$ & 3.1 & 0.5 & 3.1 & 0.5 & 2.2 & 0.4 & 2.0 & 0.4 & 1.2 & 0.5 & 1.5 & 0.5 & 3.7 & 0.4 & 23.4 & 1.9 & 27.7 & 0.5 & 7.6 & 0.4 \\
\hline $2.80-3.00$ & 5.5 & 0.5 & 4.8 & 0.5 & 3.8 & 0.4 & 3.3 & 0.4 & 2.3 & 0.4 & 4.1 & 1.1 & 16.8 & 1.6 & 23.2 & 0.5 & 2.4 & 0.4 & 1.4 & 0.3 \\
\hline $3.00-3.20$ & 5.1 & 0.6 & 5.5 & 0.7 & 4.6 & 0.5 & 4.6 & 0.5 & 8.4 & 1.7 & 20.0 & 2.8 & 24.5 & 1.2 & 2.2 & 0.3 & 1.6 & 0.2 & 1.7 & 0.2 \\
\hline $3.20-3.40$ & 5.7 & 0.7 & 5.3 & 0.6 & 5.7 & 0.7 & 9.8 & 1.7 & 26.5 & 3.8 & 25.9 & 1.5 & 5.1 & 0.4 & 1.9 & 0.3 & 1.8 & 0.2 & 1.6 & $0 . ?$ \\
\hline $3.40 \cdot 3.60$ & 16.0 & 2.2 & 15.6 & 2.5 & 22.9 & 1.9 & 32.2 & 3.8 & 23.4 & 1.8 & 4.1 & 0.3 & 2.0 & 0.4 & 2.5 & 0.4 & 1.8 & 0.4 & 1.6 & 0.3 \\
\hline $3.60-3.80$ & 39.7 & 5.1 & 40.2 & 5.2 & 35.6 & 4.3 & 24.1 & 1.9 & 2.7 & 0.3 & 1.6 & 0.3 & 1.7 & 0.4 & 2.2 & 0.5 & 2.1 & 0.6 & 2.5 & 0.6 \\
\hline $3.80-4.00$ & 24.6 & 2.7 & 23.9 & 3.1 & 10.6 & 2.3 & 2.7 & 0.4 & 1.5 & 0.3 & 1.0 & 0.3 & 1.6 & 0.5 & 2.3 & 0.6 & 5.3 & 0.9 & 14.2 & 1.3 \\
\hline $4.00-4.25$ & 5.9 & 0.4 & 5.0 & 0.5 & 3.1 & 0.4 & 1.9 & 0.3 & 1.3 & 0.3 & 1.4 & บ. 3 & 2.2 & 0.6 & 5.8 & 0.9 & 46.0 & 0.7 & 118.4 & 1.7 \\
\hline $4.25 \cdot 4.50$ & 5.3 & 0.4 & 5.6 & 0.4 & 3.4 & 0.3 & 2.6 & 0.3 & 1.8 & 0.3 & 2.0 & 0.4 & 4.7 & 0.7 & 42.6 & 0.6 & 172.9 & 2.7 & 179.8 & 2.7 \\
\hline $4.50-4.75$ & 5.3 & 0.5 & 5.5 & 0.4 & 4.1 & $c .3$ & 2.3 & 0.3 & 2.5 & 0.4 & 5.5 & 0.3 & 32.7 & 0.7 & 159.7 & 3.8 & 99.8 & 1.7 & 22.1 & 0.5 \\
\hline $4.75-5.00$ & 10.4 & 0.7 & 9.4 & 0.5 & 6.9 & 0.4 & 4.6 & 0.5 & 6.6 & 0.4 & 21.5 & 0.6 & 114.2 & 3.5 & 109.7 & 2.8 & 7.6 & 0.3 & 2.7 & 0.2 \\
\hline $5.00-5.25$ & 32.2 & 1.2 & 24.7 & 0.6 & 31.3 & 0.5 & 22.6 & 0.5 & 29.5 & 0.6 & 60.7 & 1.4 & 96.3 & 1.6 & 8.8 & 0.4 & 1.3 & 0.1 & 0.6 & 0.1 \\
\hline $5.25 \cdot 5.50$ & 137.8 & 2.3 & 111.7 & 2.9 & 129.8 & 4.4 & 88.7 & 2.2 & 75.1 & 1.3 & 62.5 & 1.1 & 10.9 & 0.4 & 1.2 & 0.1 & 0.3 & 0.1 & & \\
\hline $5.50-5.75$ & 393.9 & 9.8 & 312.8 & 9.6 & 317.6 & 8.7 & 209.8 & 4.8 & 63.1 & 1.2 & 12.7 & 0.3 & 0.9 & 0.2 & 0.1 & 0.1 & & & & \\
\hline $5.75 \cdot 6.00$ & 703.5 & 18.5 & 618.8 & 14.7 & 361.6 & 7.4 & 176.1 & 4.1 & 10.2 & 0.5 & 0.9 & 0.1 & & & & & & & & \\
\hline $6.00-6.25$ & 403.4 & 12.1 & 359.8 & 8.7 & 89.9 & 2.6 & 24.3 & 1.5 & 0.4 & 0.1 & & & & & & & & & & \\
\hline $6.25-6.50$ & 56.9 & 4.1 & 32.2 & 2.5 & 3.2 & 0.2 & 0.9 & 0.1 & 0.1 & 0.1 & & & & & & & & & & \\
\hline $6.50-6.75$ & 3.5 & 0.2 & 2.1 & 0.2 & 1.5 & 0.1 & 0.6 & 0.1 & & & & & & & & & & & & \\
\hline$=75-7.00$ & 1.7 & 0.2 & 1.4 & 0.1 & & & & & & & & & & & & & & & & \\
\hline $7.00-7.25$ & 0.6 & 0.1 & & & & & & & & & & & & & & & & & & \\
\hline
\end{tabular}


- 0 M MNNT-

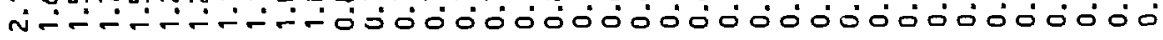

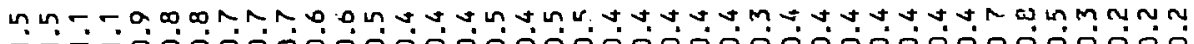

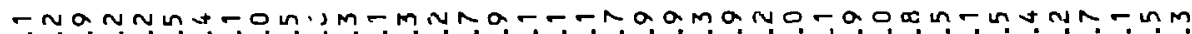

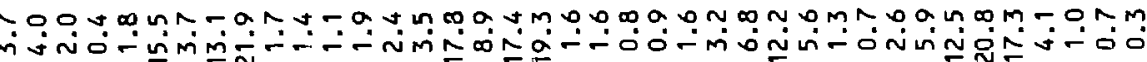



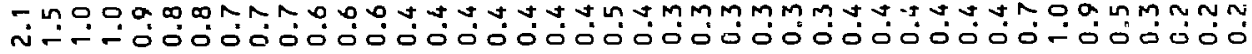

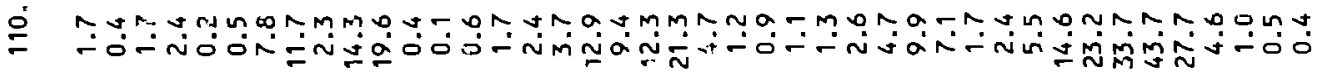


$\because \because 0$ -

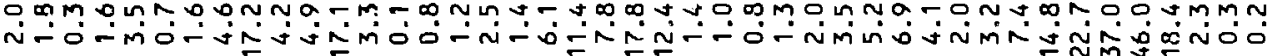

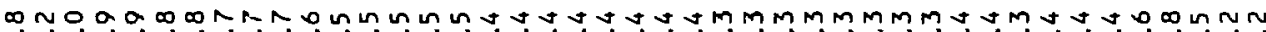

0

R

$\stackrel{\circ}{\circ}$

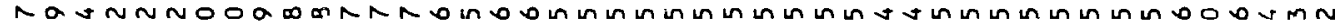

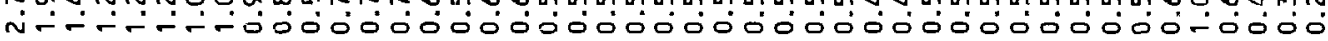



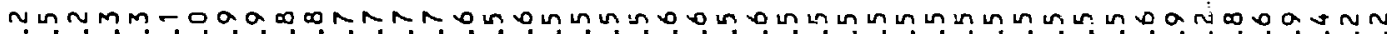
N- - - -

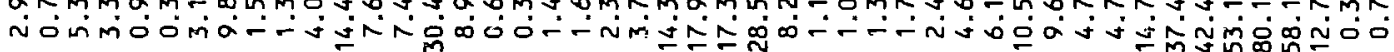

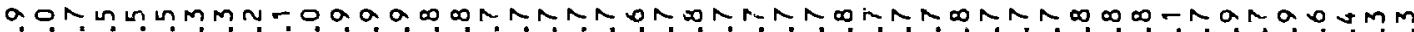

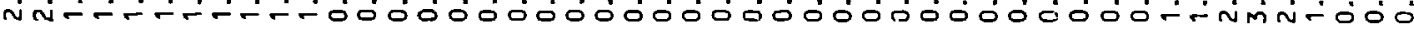

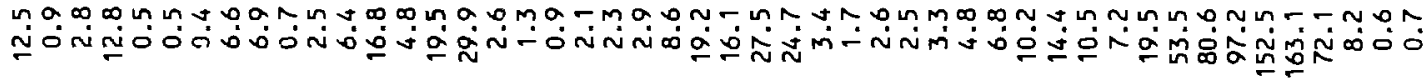

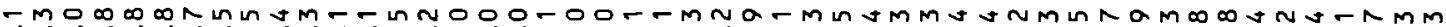
miñ

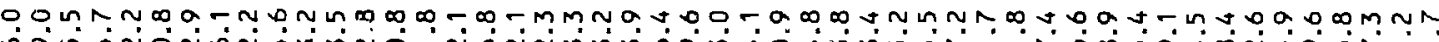

愚



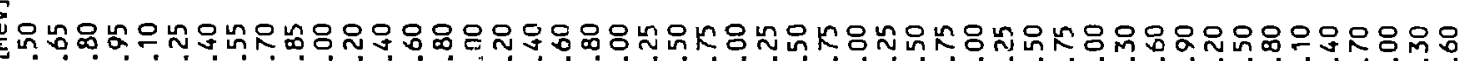
”̈

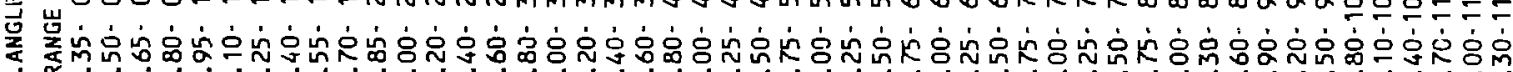
\& 
neutrons were tallied by energy and angle according to the reaction types that created them: single elastic, double elastic, elastic-inelastic, single inelastic, double inelastic, and inelastic-elastic processes. As can be seen in the spectra shown in the Appendix this simuiation does not stand up to realicy. In many cases the scale bf the inelastic portior is grossly too low; for ${ }^{11} \mathrm{~B}: 2=10 \mathrm{MeV}$ not eren the shape of the spectrum is properly simulated. Consequently, also the simulated multiple scattering corrections are expected to be equally poor. Since an improvement of the cross section library ENDF/B-IV was not within the scope of this work, the following procedure is believed to give a first order improvement: the elastic and the inelastic portions of the simulated spectra were so adjusted that their integrals agreed with those of the corresponding portions of the measured spectra, changing the original simulation (shown as dotted curves in the figures of the Appendix) to the piecewise adjusted solution (dashed curves). The same adjustment factors were then applied to the corrections, i.e., their energy shape remained unchanged. Because of the sometimes very poor agreement in shape, the corrections were then done additively rather than in the usual multiplicative way.

\section{RESULTS}

\section{A. Double Differential Cross Sections}

Tables $V$ through VII give the multiple scattering corrected double differential cross sections. Because the quality of the experiment is much better than that of the correction and a remeasurement is unlikely because of its complexity, it seems prudent to present both corrected and uncorrected data in numerical form. The former can be used to improve the 
data base for a better multiple scattering correction which $\mathrm{c}$ n then be applied to the uncorrected data. It is interescing to note that for both energies and both isotopes, practically all levels that can be excited are actually seen in the spectra, whereas only a. few have been seen before 7.8 in neutron scattering experiments. This is possible because of the much mr,re intense neutron source used in this experiment. However, no attempt was made to evaluate the inelastic differential cross sections of the individual. levels because the multiple scattering correction is so unreliable.

\section{B. Differential Elastic Cross Sections}

The multiple scattering correction of the elastically scattered neutrons is expected to be quite reliable. Therefore, differential cross sections for the elastic scattering were extracted. In the case of ${ }^{10} \mathrm{~B}$, scattering involving the first excited state at $0.72 \mathrm{MeV}$ could not be separated from the elastic scattering. Therefore, ia Table IX we give cross sections of the highest energy peak rather than elastic cross sections.

In addition to the measured data, zero-degree and integrated cross sections as obtained by Legendre fitting (see Sec. C) are included in this table. In addition we have listed Wick's limit $\sigma_{W}$, the lower limit for the elastic $0^{\circ}$ cross section. Its center-of-mass value is derived from the total reaction cross section $\sigma_{\mathrm{T}}$ by

$$
\frac{\mathrm{d} \sigma}{\mathrm{d} \Omega}\left(0^{0}\right) \geq \sigma_{W}=\left(\mathrm{k} \frac{\sigma_{T}}{4 \pi}\right)^{2}
$$


TABLE IX

ABSOLUTE DIFFERENTIAL LASORATORY CROSS SECTIONS OF

THE HIGHEST ENERGY PEAK

\begin{tabular}{|c|c|c|c|c|c|c|c|c|}
\hline \multirow{2}{*}{$\begin{array}{l}\text { Isotope } \\
\text { Energy }\end{array}$} & \multicolumn{4}{|c|}{${ }^{10} \mathrm{~B}$} & \multicolumn{4}{|c|}{${ }^{11} \mathrm{~B}$} \\
\hline & 6.0 & $\mathrm{MeV}$ & 10.0 & $\mathrm{MeV}$ & 6.0 & $\mathrm{MeV}$ & 10.0 & $\mathrm{MeV}$ \\
\hline $\begin{array}{l}\text { ANGLE } \\
\text { [deg] }\end{array}$ & $\begin{array}{l}\text { SIGMA } \\
{[\mathrm{mb} / \mathrm{sr}]}\end{array}$ & $\begin{array}{c}\text { ERROR } \\
\text { [ } 8]\end{array}$ & $\begin{array}{c}\text { SIGMA } \\
{[\mathrm{mb} / \mathrm{sIj}}\end{array}$ & $\begin{array}{c}\text { ERROR } \\
\text { [\%] }\end{array}$ & $\begin{array}{c}\text { SIGMA } \\
{[\mathrm{mb} / \mathrm{sr}]}\end{array}$ & $\begin{array}{c}\text { ERROR } \\
{[\%]}\end{array}$ & $\begin{array}{c}\text { SIGMA } \\
{[\mathrm{mb} / \mathrm{sI}]}\end{array}$ & $\begin{array}{c}\text { ERROR } \\
{[8]}\end{array}$ \\
\hline measured & data: & & & & & & & \\
\hline 20.0 & 331.0 & 2.2 & 435.2 & 1.8 & 437.2 & 1.4 & 429.3 & 1.0 \\
\hline 25.0 & 277.8 & 2.0 & & & 369.4 & 1.3 & & \\
\hline 35.0 & 171.9 & 2.0 & 206.8 & 1.6 & 236.7 & 1.3 & 195.3 & 0.9 \\
\hline 45.0 & 98.7 & 1.7 & 94.5 & 1.6 & 132.7 & 1.3 & 91.1 & 1.0 \\
\hline 60.0 & 37.2 & 1.5 & 22.9 & 1.4 & 47.5 & 1.0 & 25.7 & 2.1 \\
\hline 75.0 & 33.8 & 1.8 & 20.4 & 1.6 & 41.8 & 1.1 & 27.2 & 1.3 \\
\hline 90.0 & 48.6 & 1.9 & 35.4 & 1.7 & 65.6 & 1.5 & 43.3 & 1.0 \\
\hline $110 . \mathrm{C}$ & 52.8 & 2.0 & 33.9 & 1.6 & 82.9 & 1.5 & 40.9 & 1.1 \\
\hline 130.0 & 45.0 & 1.8 & 17.6 & 1.7 & 83.7 & 1.0 & 21.5 & 1.6 \\
\hline 145.0 & 461 & 1.6 & 12.3 & 1.9 & 84.5 & 1.0 & 16.4 & 2.6 \\
\hline
\end{tabular}

calculated data:

$\begin{array}{cllll}0.0 & 435.9 & 667.3 & 573.3 & 631.2 \\ \text { Wick's iimit } & 414 \mathrm{a}) & 637 \mathrm{a}) & 504 & 615 \\ \text { integrated } & 927 \mathrm{mb} \text { b) } & 844 \mathrm{mb} \text { b) } & 1331 \mathrm{mb} & 876 \mathrm{mb}\end{array}$

a) using present value of the total cross section

b) according to ENDF/B-V, inelastic scattering from the first excited state $(0.72 \mathrm{MeV})$ contributes 3 at $6 \mathrm{MeV}$ and $1 \%$ at $10 \mathrm{MeV}$ to this integrated value 
where $k$ is the center-of-mass wave number of the incoming neutron. Because of the quadratıc dependence on $\sigma_{\mathrm{T}}$, uncertainties in this quantity affect $\sigma_{\mathrm{W}}$ very strongly. In our case we took $\sigma_{\mathrm{T}}$ of ${ }^{1{ }^{1} \mathrm{~B}}$ from Reference 9 and that of $10 \mathrm{~B}$ from our present results, diminishing the effect of the scale uncertainty.

\section{Integrated Cross Sections}

The angular distributions of the elergy integrated spectra are given in Table $\mathrm{X}$. These data contain extrapolations to zero neutron energy. To account for events below the neutron detection bias, the following simple procedure was believed to be good enough considering the poor quality of the multiple scattering correction: it was assumed that the spectrum below the curoff at $0.35 \mathrm{MeV}$ was in the average as intense as the spectrum between 0.35 and $2.0 \mathrm{MeV}$. By assigning a 508 error to this extrapolation most eventualities should be taken care of.

The integration of the double differential cross sections over both the energy of the emitted neutrons and the solid angle was done in two steps. First, the cross section of the highest energy peak was converted into the center-of-mass system and integrated over the solid angle in this system, and then, the remaining spectrum was integrated over the energy and over the solid angle in the laboratory system.

The integrations over the solid angle were done by fitting the angular distributions by means of Legendre polynomials $\mathrm{P}_{i}$ so that the integrated cross section was obtained from the first coefficient $A_{0}$ after multiplying by $4 \pi$. 


\section{TABLE $X$}

ABSOLUTE DIFFERENTIAL NEUTRON EMISSION CROSS SECTIONS

\begin{tabular}{|c|c|c|c|c|c|c|c|c|}
\hline \multirow{2}{*}{$\begin{array}{l}\text { Isotope } \\
\text { Energy }\end{array}$} & \multicolumn{4}{|c|}{${ }^{10} \mathrm{~B}$} & \multicolumn{4}{|c|}{${ }^{11} \mathrm{~B}$} \\
\hline & \multicolumn{2}{|c|}{$6.0 \mathrm{MeV}$} & \multicolumn{2}{|c|}{$10.0 \mathrm{MeV}$} & \multicolumn{2}{|c|}{$6.0 \mathrm{MeV}$} & \multicolumn{2}{|c|}{$10.0 \mathrm{MeV}$} \\
\hline $\begin{array}{c}\text { ANGLE } \\
\text { [deg] }\end{array}$ & $\begin{array}{c}\text { SIGMA } \\
{[\mathrm{mb} / \mathrm{sr}]}\end{array}$ & $\begin{array}{c}\text { ERROR } \\
{\left[\begin{array}{c}\text { fo } \\
\end{array}\right]}\end{array}$ & $\begin{array}{c}\text { SIGMA } \\
{[\mathrm{mb} / \mathrm{sr}]}\end{array}$ & $\begin{array}{c}\text { ERROR } \\
{[z]}\end{array}$ & $\begin{array}{c}\text { SIGMA } \\
{[\mathrm{mb} / \mathrm{sr}]}\end{array}$ & $\begin{array}{c}\text { ERROR } \\
{[q]}\end{array}$ & $\begin{array}{c}\text { SIGMA } \\
{[\mathrm{mb} / \mathrm{sr}]}\end{array}$ & $\begin{array}{c}\text { ERROR } \\
{\left[\frac{q}{z}\right]}\end{array}$ \\
\hline \multicolumn{9}{|c|}{ measured data: } \\
\hline 20.0 & 357.8 & 2.0 & 490.3 & 1.6 & 489.3 & 1.7 & 521.8 & 1.1 \\
\hline 25.0 & 306.2 & 1.9 & & & 422.0 & 1.9 & & \\
\hline 35.0 & 195.8 & 1.9 & $25 i .8$ & 1.5 & 279.7 & 1.8 & 262.1 & 1.1 \\
\hline 45.0 & 118.8 & 1.7 & 3.27 .9 & 1.5 & 171.2 & 1.9 & 145.5 & 1.8 \\
\hline 60.0 & 52.9 & 1.9 & 52.2 & 2.1 & 80.1 & 2.9 & 63.4 & 2.1 \\
\hline 75.0 & 48.6 & 2.0 & 46.2 & 2.1 & 71.3 & 2.9 & 61.7 & 1.8 \\
\hline 90.0 & 60.5 & 2.0 & 50.1 & 2.3 & 90.8 & 1.9 & 75.7 & 1.6 \\
\hline 110.0 & 61.7 & 1.9 & 54.7 & 2.3 & 104.2 & 1.5 & 73.2 & 1.7 \\
\hline 130.0 & 52.6 & 1.7 & 36.4 & 2.7 & 105.0 & 1.3 & 53.6 & 2.1 \\
\hline 145.0 & 53.0 & 2.6 & 32.5 & 2.6 & 103.5 & 1.5 & 52.3 & 2.6 \\
\hline \multicolumn{9}{|c|}{ calculated data: } \\
\hline 0.0 & \multicolumn{2}{|c|}{469.4} & \multicolumn{2}{|c|}{730.5} & \multicolumn{2}{|c|}{634.1} & \multicolumn{2}{|c|}{738.3} \\
\hline integrated & \multicolumn{2}{|c|}{$1097 \mathrm{mb}$} & \multicolumn{2}{|c|}{$1187 \mathrm{mb}$} & \multicolumn{2}{|c|}{$1685 \mathrm{mb}$} & \multicolumn{2}{|c|}{$1396 \mathrm{mb}$} \\
\hline
\end{tabular}


The advantage of the two step procedure lies in the smaller number of coefficients needed in the center-of-mass presentation of the elastic cross section, so that for the limited number of experimental points the fit (and its extrapolation to $0^{\circ}$ and $180^{\circ}$ ) becomes more reliable. After removal of the strongly forward peaked elastic cross sections, the remaining data can be fitted even in the laboratory system with only a few coefficients.

Table XI collects all Legendre coefficients obtained in the presert work. For convenience, the Legendre coefficients for describing the angular distribution of the total neutron emission in the laboratory system are also given. The latter fit was obtained under the following three constraints: $A_{0}$ was fixed to the sum of the first coefficient of the elastic and that of the inelastic distribution, and the zero-degree and the 180-degree cross sections were taken to be the sum of the corresponding (extrapolated) values of the partial fits.

V. UNCERTAINTIES

A. Energy and Angle

The structure in the energy dependence and the rather strong angular dependence of the cross sections require a good knowledge both of the mean neutron energy and the scattering angle. For this work, the spread of the incoming energy was assumed to be rectangular, and the angular spread was disregarded. The incoming neutron energy is uncertain by $0.03 \mathrm{MeV}$, the zero degree direction by $0.4^{\circ}$. Compared to the latter, the individual uncertainty of each angle is unimportant $\left(0.1^{\circ}\right)$. 


\section{TABLE XI}

LEGENDRE COEFFICIENTS FOR THE PRESENTATION OF THE ANGULAR DISTRIBUTIONS

\section{Isotope}

Energy

\author{
${ }^{10} \mathrm{~B}$ \\ $6 \mathrm{MeV} 10 \mathrm{MeV}$
}

\author{
${ }^{11} \mathrm{~B}$ \\ $6 \mathrm{MeV} 10 \mathrm{MeV}$
}

Elastic (C.M.):

$\begin{array}{rrr}\mathrm{A}_{0} & 73.8 & 67.2 \\ \mathrm{~A}_{1} & 60.5 & 104.9 \\ \mathrm{~A}_{2} & 94.9 & 126.9 \\ \mathrm{~A}_{3} & 84.1 & 127.1 \\ \mathrm{~A}_{4} & 43.9 & 79.5 \\ \mathrm{~A}_{5} & 2.2 & 16.9 \\ \mathrm{~A}_{6} & & 15.4 \\ \mathrm{~A}_{7} & & 6.3 \\ \mathrm{~A}_{8} & & 5.5\end{array}$

$\begin{array}{rr}105.9 & 69.7 \\ 65.6 & 98.4 \\ 138.4 & 117.7 \\ 116.0 & 126.2 \\ 51.2 & 80.9 \\ 3.3 & 17.3 \\ & 11.6 \\ & 4.6 \\ & 2.3\end{array}$

Nonelastic (1ab):

$A_{0}$
$A_{1}$
$A_{2}$
$A_{3}$
$A_{4}$
$A_{5}$

Total Enission (lab):

$\begin{array}{rr}13.5 & 27.3 \\ 10.1 & 13.2 \\ 4.6 & 10.7 \\ 2.1 & 4.2 \\ 2.3 & 6.9 \\ 0.9 & 0.8\end{array}$

87.3

81.6

104.0

101.7

69.8

20.2

4.0

1.0
94.5

126.0

146.3

150.0

119.2

45.3

23.9

11.9

10.6

2.8

$\begin{array}{rr}28.2 & 41.4 \\ 15.6 & 18.1 \\ 6.9 & 24.6 \\ 5.5 & 12.3 \\ 1.2 & 10.1 \\ 3.2 & 0.6\end{array}$

$134.1 \quad 111.1$

$95.6 \quad 124.9$

$147.7 \quad 149.2$

$143.2 \quad 153.6$

$84.9 \quad 119.9$

$26.5 \quad 43.9$

$2.1 \quad 20.2$

9.5

6.0 


\section{B. Individual Cross Section Errors}

The final error given with each data point of the secondary neutron spectra consists of the "subined counting statistics of the individual specira plus curicributions of various corrections:

- subtraction of white (time-uncorrelated) time-of-flight background

- subtraction of the spectrum of the admixed isotope and other impurities (hydrogen in $10 \mathrm{~B}$ )

- multiple scattering correction.

For each data point these corrections are uncorrelated and, therefore, their error contributions (see Table XII) were added quadratically. However, within each spectrum these error contributions are correlated (to a degree depending on the $t_{j}$ pe of correction), so that combining uncertainties of individual energy bins quadratically will give a distorted combined error.

To avoid an even stronger masking of the truly random errors, the uncertainty in the neutron detection efficiency which is also strongly correlated is not included. At the secondary neutron energies at which the cross section standard was used $(4.0$ and $6.7 \mathrm{MeV}$ for the $6-$ and $10 \mathrm{MeV}$ data, respectively) this uncertainty is even zero. For energies down to about $0.65 \mathrm{MeV}$ the uncertainty can best be expressed ${ }^{5}$ by $28 / 10 \mathrm{MeV}$. Below that energy it increases strongly due to the steepness of the efficiency curve and the increasing dependence on the stability of the bias. The uncertainty in the dead-time correction which is less than 0.1 was not folded in either. 


\title{
TABLE XII
}

PERCENT EFFECT OF CORRECTIONS ON THE INTEGRATED VALUES OF THE ELASTIC, NONELASTIC, AND TOTAL EMISSION CROSS SECTIONS.

\section{Isotope}

Energy

Hydrogen Contamination ( $\left.108^{a}\right)$ :
elastic
nonelastic
cotal emission

Monte Carlo Correction ${ }^{b}\left(20 q^{a}\right)$ :

elastic

total emission

\author{
$10 \mathrm{~B}$ \\ $6 \mathrm{MeV} 10 \mathrm{MeV}$ \\ $1.6 \quad 0.6$ \\ $20.8 \quad 11.9$ \\ $5.2 \quad 3.5$
}

$16.3 \quad 16.4$

$15.0 \quad 14.3$

Extrapolation of Energy $\operatorname{Spectra}^{c}\left(50{ }^{a}\right)$ : nonelastic total emission

$8.8 \quad 8.3$

7. 56.2

$6 \mathrm{MeV} \quad 10 \mathrm{MeV}$

$\begin{array}{llll}76 & 3.6 & 8.4 & 2.8 \\ 1.2 & 1.0 & 1.8 & 1.0\end{array}$

Extrapolation of Angular Range ${ }^{d}$

typically 0.58 , up to 1.18

\begin{abstract}
a) this fraction of the correction has been added quadratically to the final random orrors of the individiual points b) consisting of multiple scattering, flux attenuation, and geometry
corrections

c) correcting for the $0.35 \mathrm{MeV}$ cut-off of the neutron emission spectra

d) effect on $\dot{A}_{0}$ of the Legendre fit when the zero degree value and the 180 degree value are varied within a reasonable range and the number of coefficients used in the fit is changed by one

C. Scale Uncertainty

Table XII compiles the contributions to the scale error. The common scale error is $0.7 \%$ and consists of the uncertainty in the number of hydrogen nuclei and in the cross section of the reference sample. The inclusion of the latter into the common scale error is justified by the closeness of the two energies. No substantial systematic error contribution is expected from using polyethelene
\end{abstract}


instead of hydrogen itself. The close agreement in shape and mass of the polyethylene and the carbon foam samples diminishes background subtraction problems. In addition, the flux depression and multiple scattering in both samples were corrected for by Monte Carlo calculation.

The other contributions listed in Table XIII except for the last one are identical either for the two energies or the two samples. Neglecting the correlations, the combined scale errors of the four measurements are between $1.9 \%$ and 2.28 .

D. Uncertainties of Integrated Data

The uncertainties given in Tables IX and $X$ were obtained by adding quadratically the individual errors disregarding any correlation. To provide more information on possible correlations, Table XII lists the effect of the corrections on the integrated data together with the percentage which was used for the quadratic addition to the random error of the individual points. More details are given in the footnotes of the table.

\section{VI, DISCUSSION}

Except for data near $14 \mathrm{MeV}^{10,11}$ no previous data on neutron emission spectra for these targets are known. Only scattering from elastic and some inelastic levels has been reported in the energy range covered in this experiment:

Glendinning et al. ${ }^{12}$ from 8 to $14 \mathrm{MeV}$, Cookson and Locke ${ }^{13}$ at $\zeta .72 \mathrm{MeV}$, and white et al. ${ }^{14}$ and Knox et al. 15 , respectively, below $8 \mathrm{MeV}$. The present data at $10 \mathrm{MeV}$ are generally lower by 5 to $10 \%$ than previous data ${ }^{12,13}$. The agreement in the shape is moderate to poor except for ${ }^{11} \mathrm{~B}$.

There, an astoundingly good agreement in shape is found with the data of Glendinning et al. ${ }^{12}$ when a difference in the $0^{\circ}$ direction of $0.7^{\circ}$ is taken into account. 
1. Energy Independent Contributions:

1.1 Standard

mass (we: ght, purity)

cross $s$ ition a

1.2 Sample

mass (weight, purity)
${ }^{10} \mathrm{~B}$

$11 \mathrm{~B}$

$<0.58$

$<0.53$

$<18$

$<18$

2. Energy Dependent Contributions:

$6 \mathrm{MeV}$

$10 \mathrm{MeV}$

2.1 Standard

peak evaluation $b$

0.58

0.98

normalization $c$

Monte Carlo correction d

geometry e

2.2 Samples

Monte Carl, correction $f$
$<0.58$

0.78

1.08
$<0.58$

1.18

1.08

a) uncertainty in the ${ }^{1} \mathrm{H}(\mathrm{n}, \mathrm{n}){ }^{1} \mathrm{H}$ reference cross section at 6 and $10 \mathrm{MeV}$ is expected to be so strongly correlated that it can be assumed to be "energy independent"

b) consists of the corioined statistical errors, the error in the deadtime correction and in the background subtraction

c) calibration of the experimental set-up using a detector with a given efficiency curve in a given geometry; it depends on the evaluation of the monitor counts and the beam charge collection

d) the Monte Carlo simulation corrects for flux attenuation, multiple scattering, and finite sample size

e) difference in the positioning of the sample with regard to the neutron source and the detector; within one angular distribution this uncertainty is uncorreliced, but not for the reference measurement

f) uncertainties connected with the flux depression in the sample are clearly correlated within each angular distribution; in the present case this systematic error contribution was not extracted from the total uncertainty of this correction 
Table XIV compares the integrated cross sections with the values of ENDF/B-V and some recent total cross section measurements ${ }^{9}$. At these lower energies the total cross sections of $11 \mathrm{~B}$ are also too low in ENDF/B-V, analogous to our findings ${ }^{3}$ at $14 \mathrm{MeV}$.

A comparison with the data of Auchampaugh et al. ${ }^{9}$ gives a very good agreement for ${ }^{11} \mathrm{~B}$ where there is no or only little difference between the integrated total emission closs section and the total cross section. However, for ${ }^{10} \mathrm{~B}$ it suggests that the present ${ }^{10} \mathrm{~B}$ data are low by about 2.88 if the charged particle data taken from FNDF/B-V are correct. This difference itself is only slightly outside the combined scale errors. However, the ratio between the ${ }^{10} \mathrm{D}$ and ${ }^{11} \mathrm{~B}$ cross sections is much more accurate because of the strong correlation in the scale errors (see Table XIII) so that there appears to be a systematic discrepency.

On the other hand, a comparison of the integrated olastic cross sections : $10 \mathrm{MeV}$ with those of Giendinning et al. ${ }^{12}$ suggests that the scale of our ${ }^{10} \mathrm{~B}$ data is high compared to that of ${ }^{11} \mathrm{~B}$. Also, the very good agreement of the integrated total emission data of ${ }^{10} \mathrm{~B}$ with the values given in ENDF/B-V further supports our present soluiion. Thus, the (sma11) difference between our data and tlose of Auchampaugh et al. ${ }^{9}$ is supported by other data. Possible causes for it are either the non-neutron cross sections involved or the insufficient quality of the lata bases for the Monte Carlo simulation. 


\section{COMPARISON OF INTEGRATED CROSS SECTIONS (in barns)}

\begin{tabular}{|c|c|c|c|c|c|c|c|}
\hline $\begin{array}{l}\text { Energy } \\
(\mathrm{MeV})\end{array}$ & Sample & $\exp ^{\text {Ej) }}$ & $\begin{array}{l}\text { ic } \\
\left.\text { eval }{ }^{b}\right)\end{array}$ & $\begin{array}{l}\text { Total } \\
\text { expa) }\end{array}$ & $\begin{array}{l}\text { mission } \\
\text { eval }\end{array}$ & $\begin{array}{l}\text { Total } \\
\exp ^{a 5}\end{array}$ & $\begin{array}{l}\text { aciion } \\
\text { Ref.9 }\end{array}$ \\
\hline 6.00 & ${ }^{10} \mathrm{~B}$ & $\left.0.898^{c}\right)$ & 0.951 & 1.097 & 1.096 & $1.50^{d)}$ & 1.54 \\
\hline 10.00 & ${ }^{10} \mathrm{~B}$ & $\left.0.834^{c}\right)$ & 0.931 & 1.187 & 1.196 & $1.44^{\mathrm{d})}$ & 1.49 \\
\hline 6.00 & ${ }^{11} \mathrm{~B}$ & 1.331 & 1.426 & 1.685 & 1.555 & 1.69 & 1.66 \\
\hline 10.00 & ${ }^{11} \mathrm{~B}$ & 0.876 & 0.886 & 1.396 & 1.274 & $\left.1.41^{d}\right)$ & 1.41 \\
\hline
\end{tabular}
a) this work
b) ENDF/B - V
c) cross section of unresolved excited states subtracted
d) uses ENDF/B-V values for non-neutron reactions 


\section{REFERENCES}

1. D. M. Drake, G. F. Auchampaugh, E. D. Arthur, C. E. Ragan, and P. G. Young, Nucl. Sci. Eng. 63, 401 (1977).

2. P. W. Lisowski, G. F. Auchampaugh, D. M. Drake, M. Drosg, G. Haouat, N. W. Hill, and L. Nilsson, "Cross Sections For Neutron-Induced, Neutron-Producing Reactions In ${ }^{6} \mathrm{Li}$ and ${ }^{7} \mathrm{Li}$ At 5.96 And $9.83 \mathrm{MeV}, "$ Los Alamos Scientific Laboratory report LA-8342-MS (1980).

3. M. Drosg, P. W. Lisowski, D. M. Drake, R. A. Hardekopf, and M. Muel1ner, "Cross Sections For Neutron-Producing Reactions Induced By 14.1 MeV Neutrons in ${ }^{6} \mathrm{Li},{ }^{7} \mathrm{Li},{ }^{10} \mathrm{~B},{ }^{11} \mathrm{~B}$, and Carbon, " (in preparation).

4. J. C. Hopkins and G. Breit, Nuc1. Data Tables A9, 137 (1971).

5. M. Drosg, D. M. Drake. and P. Lisowski, Nucl. Instr. Methods 176,477 (1980).

6. Los Alamos Scientific Laboratory Group X-6, "MCNP - A General Monte Carlo Code for Neutron and Photon Transport, "Los Alamos Scientific Laboratory report LA-7396-M, Rev. (November 1979).

7. F. Ajzenberg-Selove, Nuc1. Phys. A413, 1 (1984).

8. F. Ajzenberg-Selove, Nucl. Phys. A433, 1 (1985).

9. G. F. Auchampaugh, C. E. Ragan, S. Plattard, and N. W. Hill, "Neutron Total Cross-Section Measurements of ${ }^{9} \mathrm{Be}, 10,{ }^{11} \mathrm{~F}$, and ${ }^{12,13} \mathrm{C}$ from 1.0 to $14 \mathrm{MeV}$ using the ${ }^{9} \mathrm{Be}(\mathrm{d}, \mathrm{n})^{10} \mathrm{~B}$ Reaction as a "White" Neutron Source," Los Alamos Scientific Laboratory report LA-6761-MS (1977).

10. S. C. Mathur, P. S. Buchanan, and L. L. Morgan, Phys. Rev. 186, 1038 (1969).

11. M. Baba, M. Ono, N. Yabuta, T. Kikuti, and N. Hirakawa, "Scattering of 14.1-MeV Neutrons from ${ }^{10} \mathrm{~B},{ }^{11} \mathrm{~B}, \mathrm{C}, \mathrm{N}, \mathrm{O}, \mathrm{F}$ and Si," Radiation Effects, to be published.

12. S. G. Glendinning, S. El-Kadi, C. E. Nelson, R. S. Pedroni, F. O. Purser, R. L. Walter, A. G. Beyerle, C. R. Gould, L. W. Seagondollar, and P. Thambidurai, Nuc1, Sci. Eng. 80, 256 (1982).

13. J. A. Cookson and J. G. Locke, Nucl. Phys. Al46, 417 (1970).

14. R. M. White, R. O. Lane, and H. D. Knox, Nucl. Phys. A340, 13 (1980).

15. H. D. Knox, R. M. White, and R. O. Lane, Nuc1. Sci. Eng. 65, 65 (1978). 


\section{APPENDIX}

SPECTRA OF THE UNCORRECTED DOUBLE DIFFERENTIAL LABORATORY CROSS SECTIONS FOR ${ }^{10} \mathrm{~B}$ AND $11 \mathrm{~B}$ AT 6 AND $10 \mathrm{MEV}$ FOR ANGLES BETWEEN $20^{\circ}$ AND $145^{\circ}$

In Figs. A-1 through A-38, the full curves are the experimental results, the dotted curves are the straight answer of a Monte Carlo simulation using ENDF/B-IV input data and the dashed curves are the piecewise adjusted curves used in this paper for determining the multiple scattering correction. 


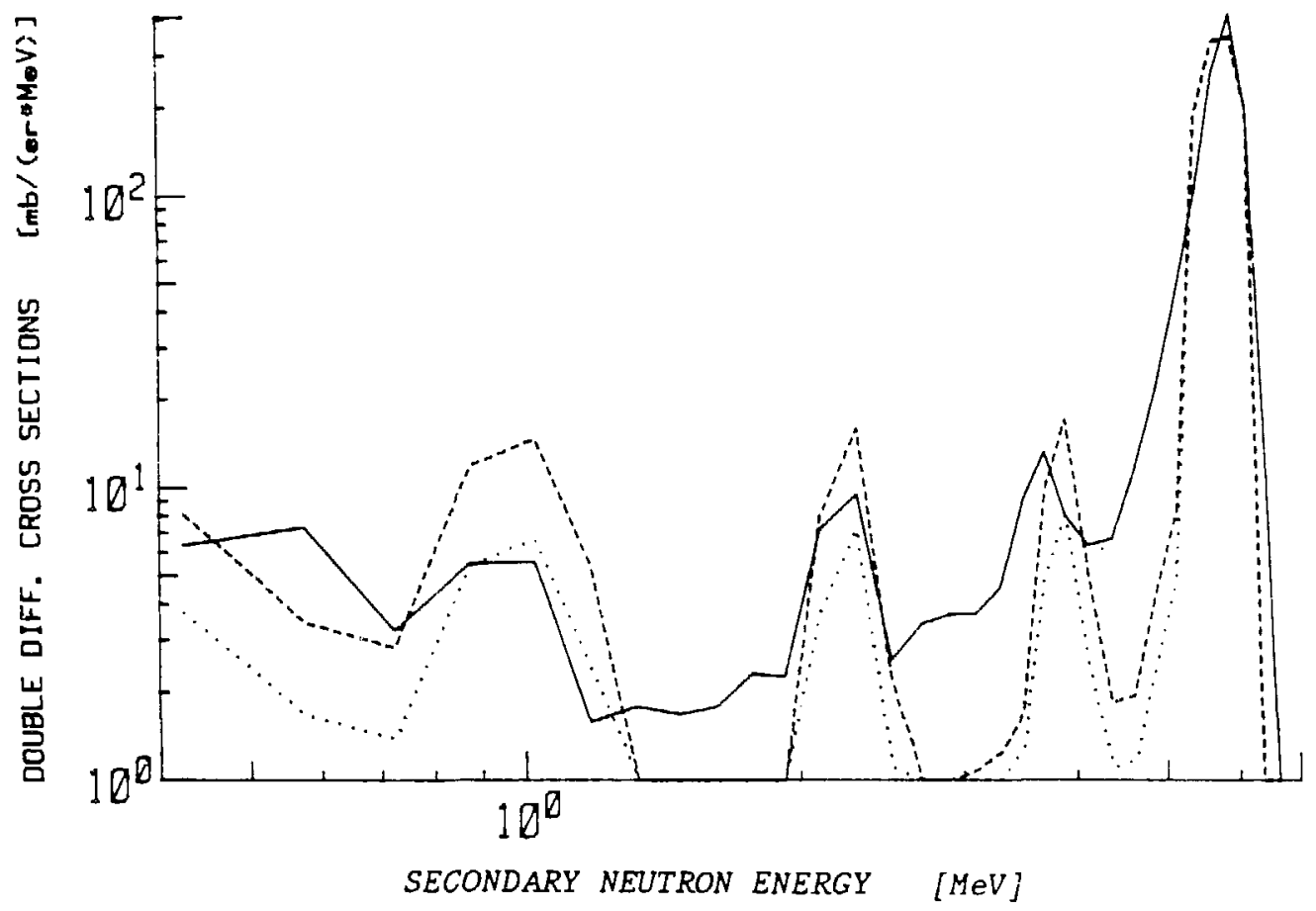

FIg. A-1. $6.0 \mathrm{MeV}$ Neutrons on Boron-10 20.0 Deg.



Fig. A-2. 6.0 MeV Neutrons on Boron-10 25.0 Deg. 


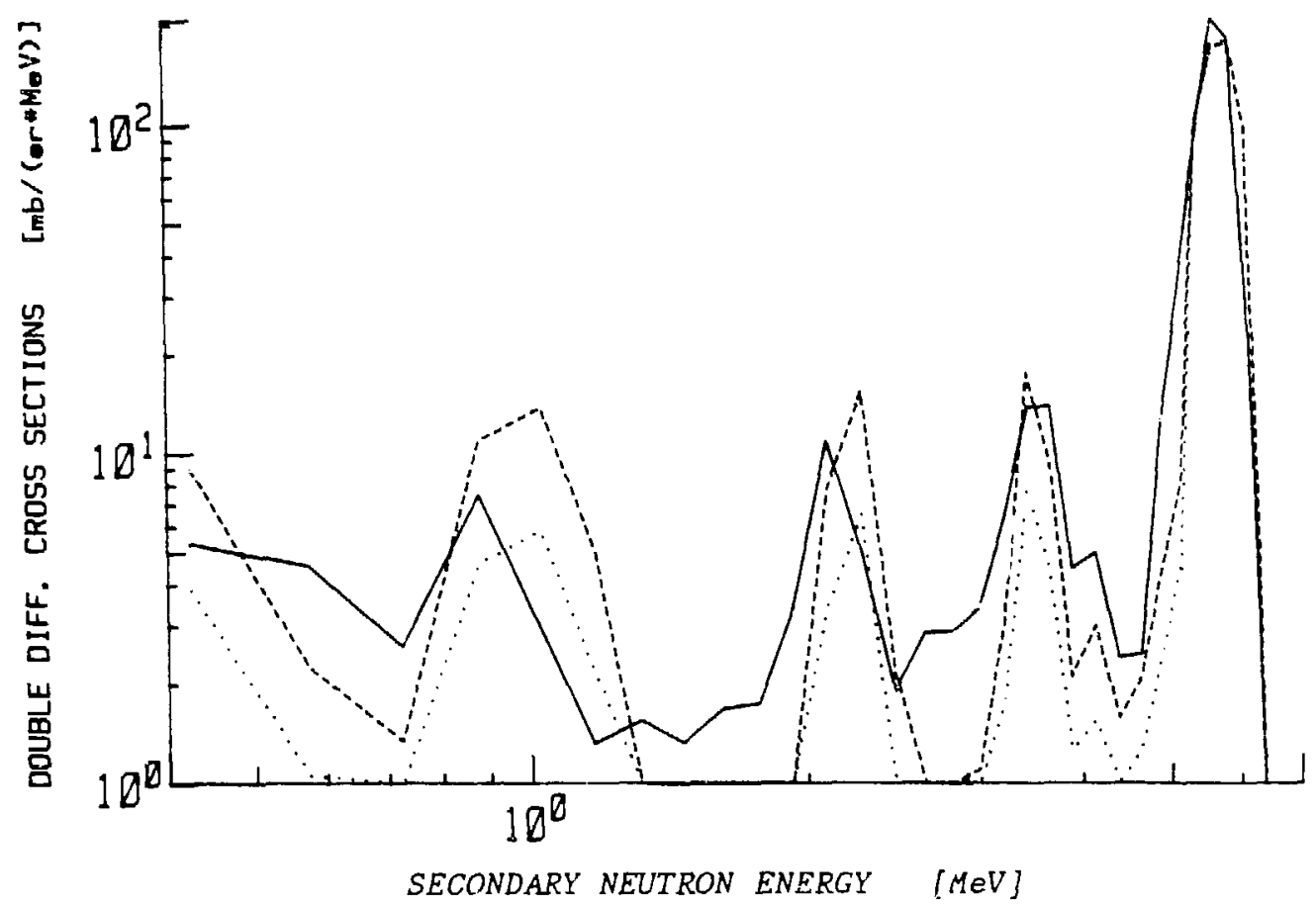

Fig. A-3. $6.0 \mathrm{MeV}$ Neutrons on Boron-10 35.0 Deg.

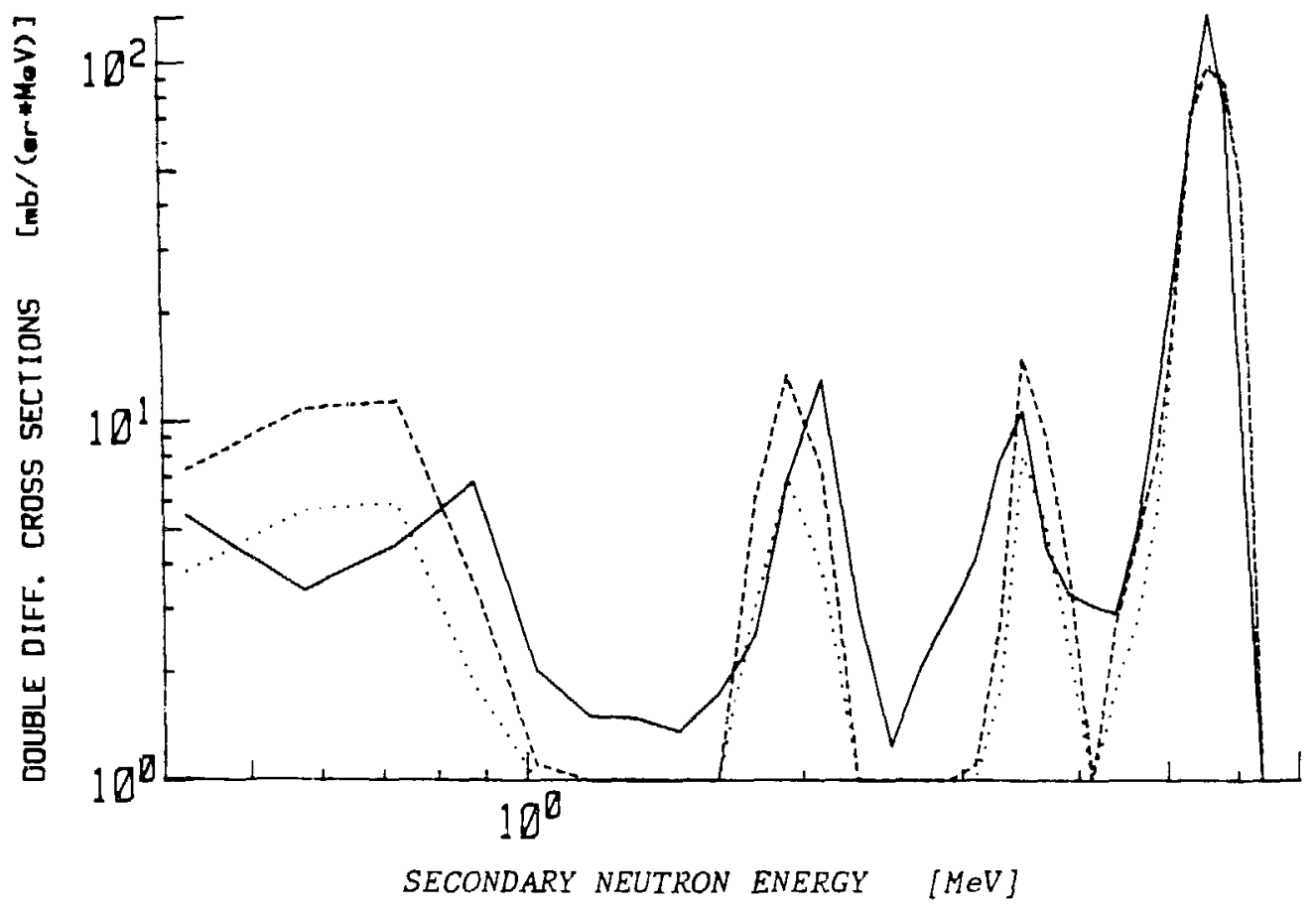

Fig. A-4. 6.0 MeV Neutrons on Boron-10 45.0 Deg. 


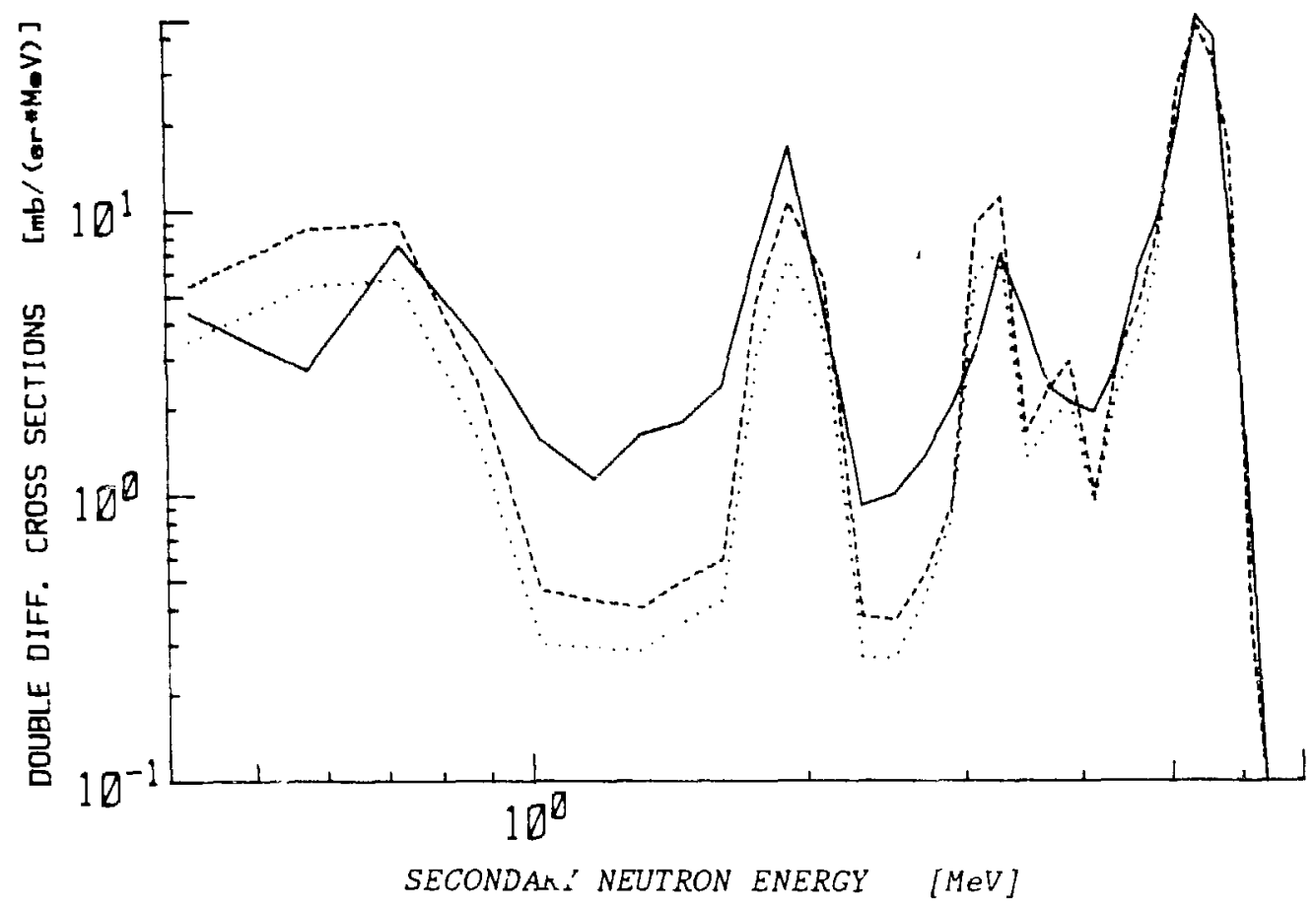

Fig. A-5. 6.0 MeV Neutrons on Boron-10 60.0 Deg.



Fig. A-6. 6.0 MeV Neutrons on Boron-10 75.0 Deg. 


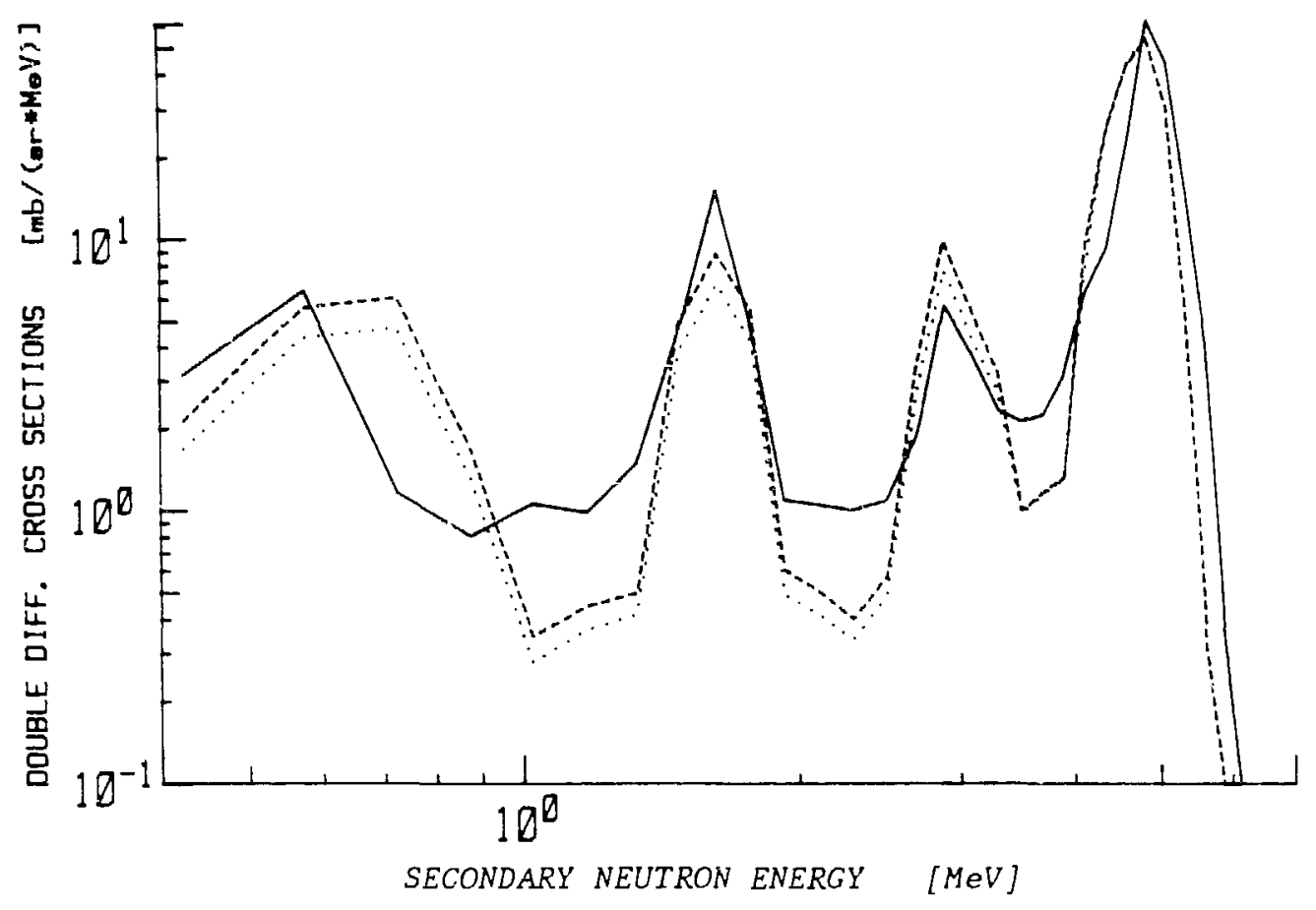

Fig. A-7. 6.0 MeV Neutrons on Boron-10 90.0 Deg.

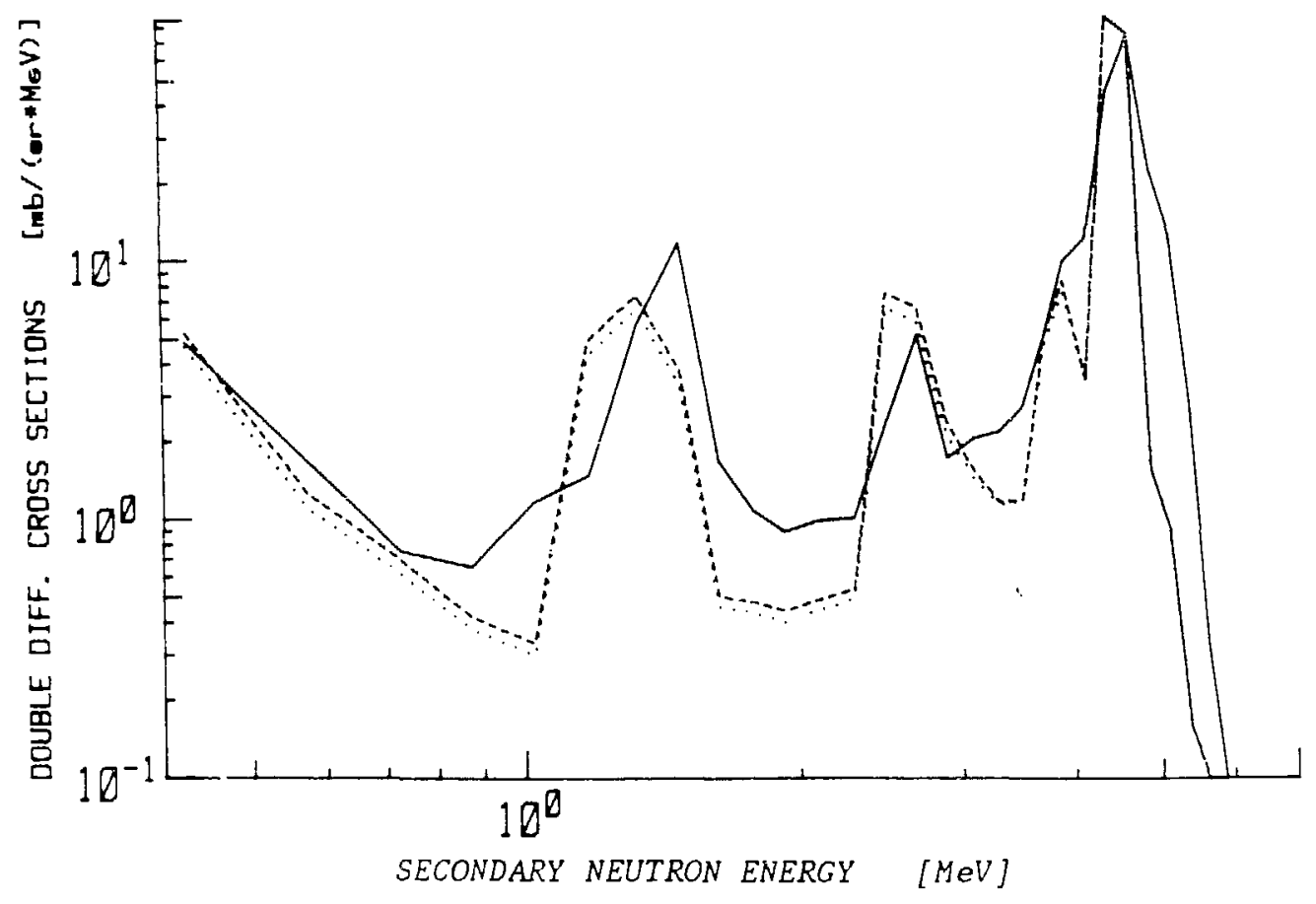

Fig. A-8. 6.0 MeV Neutrons on Boron-10 110.0 Deg. 


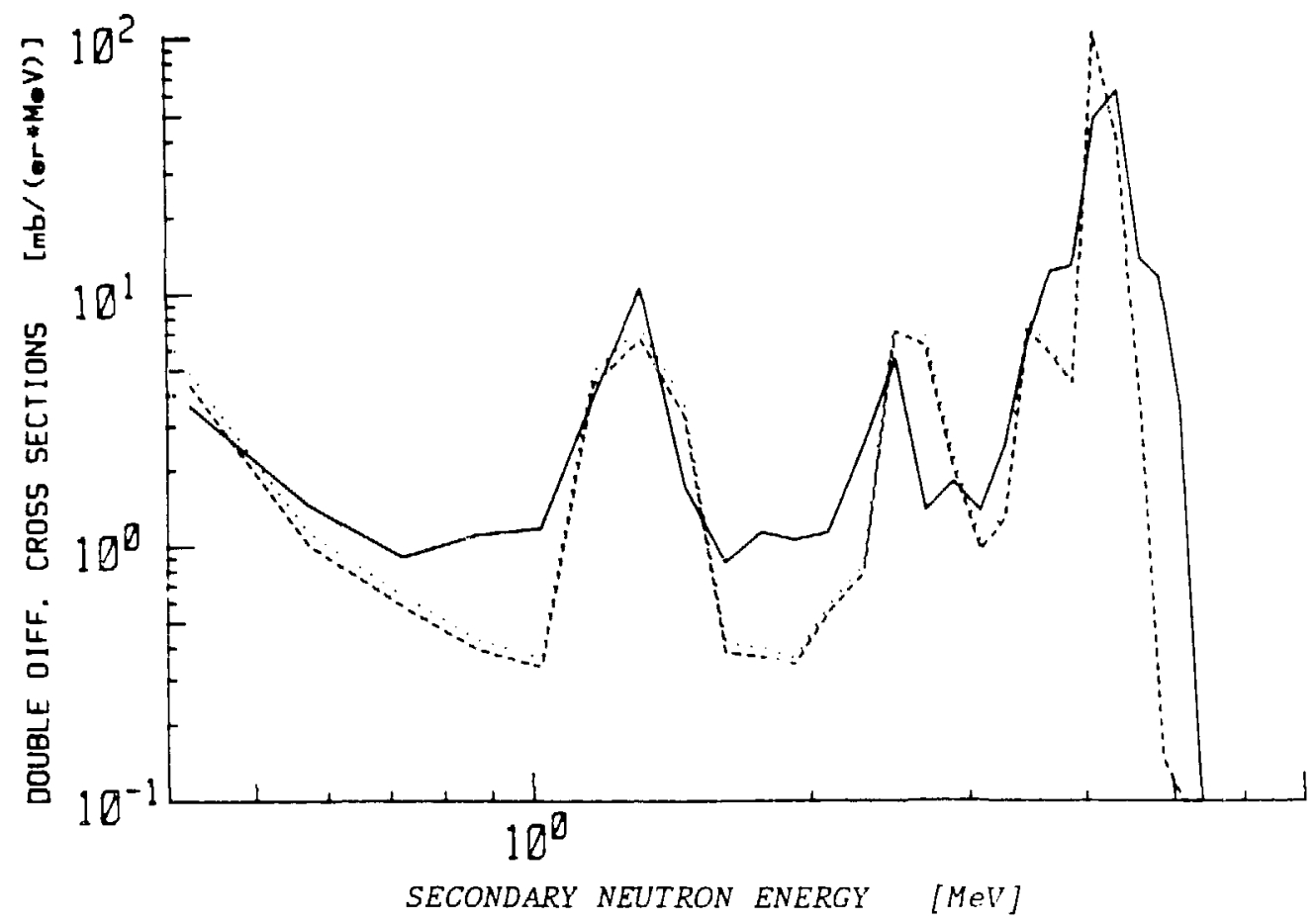

Fig. A-9. 6.0 MeV Neutrons on Boron-10 130.0 Deg.

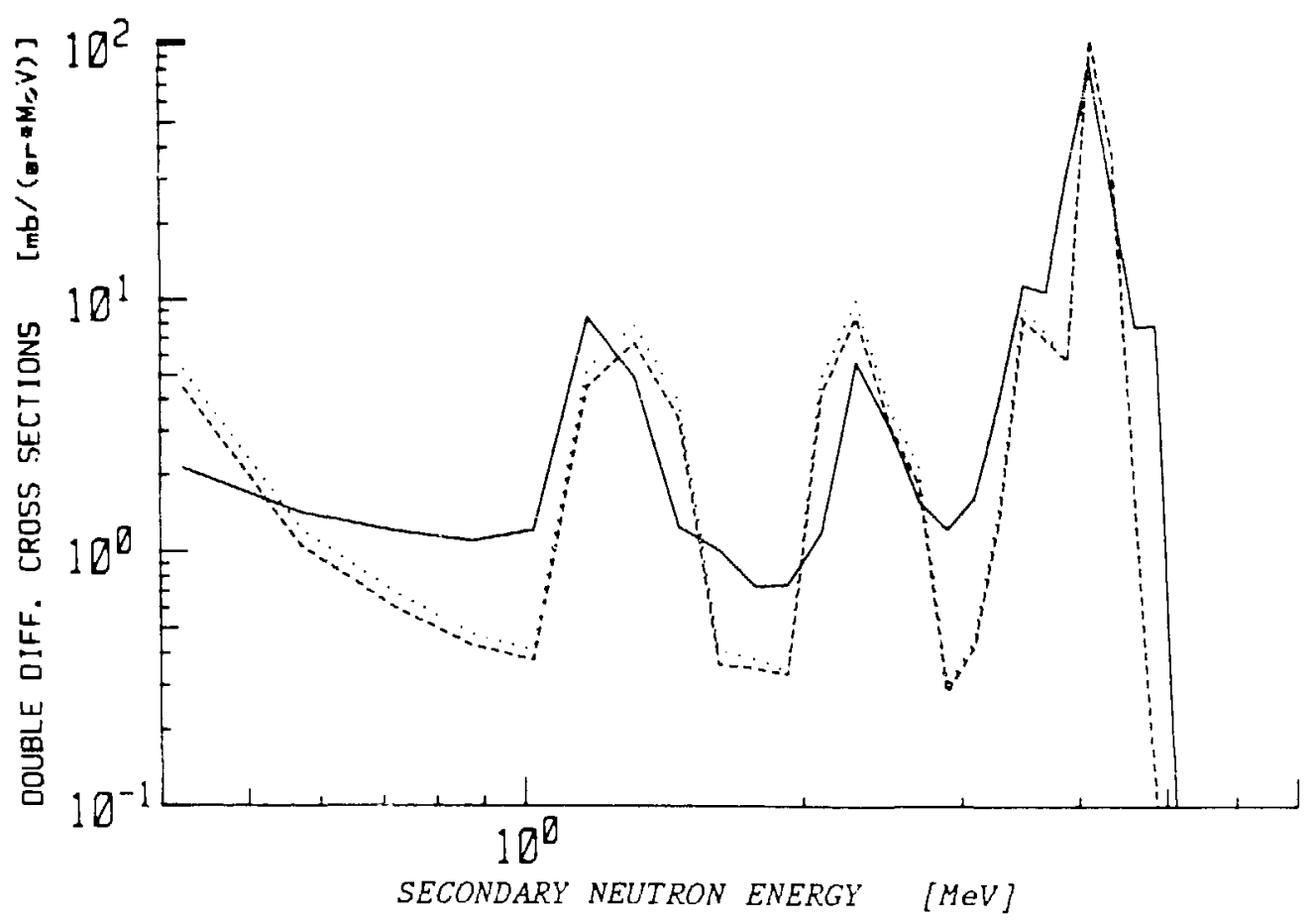

Fig. A-10. 6.0 MeV Neutrons on Boron-10 145.0 Deg. 


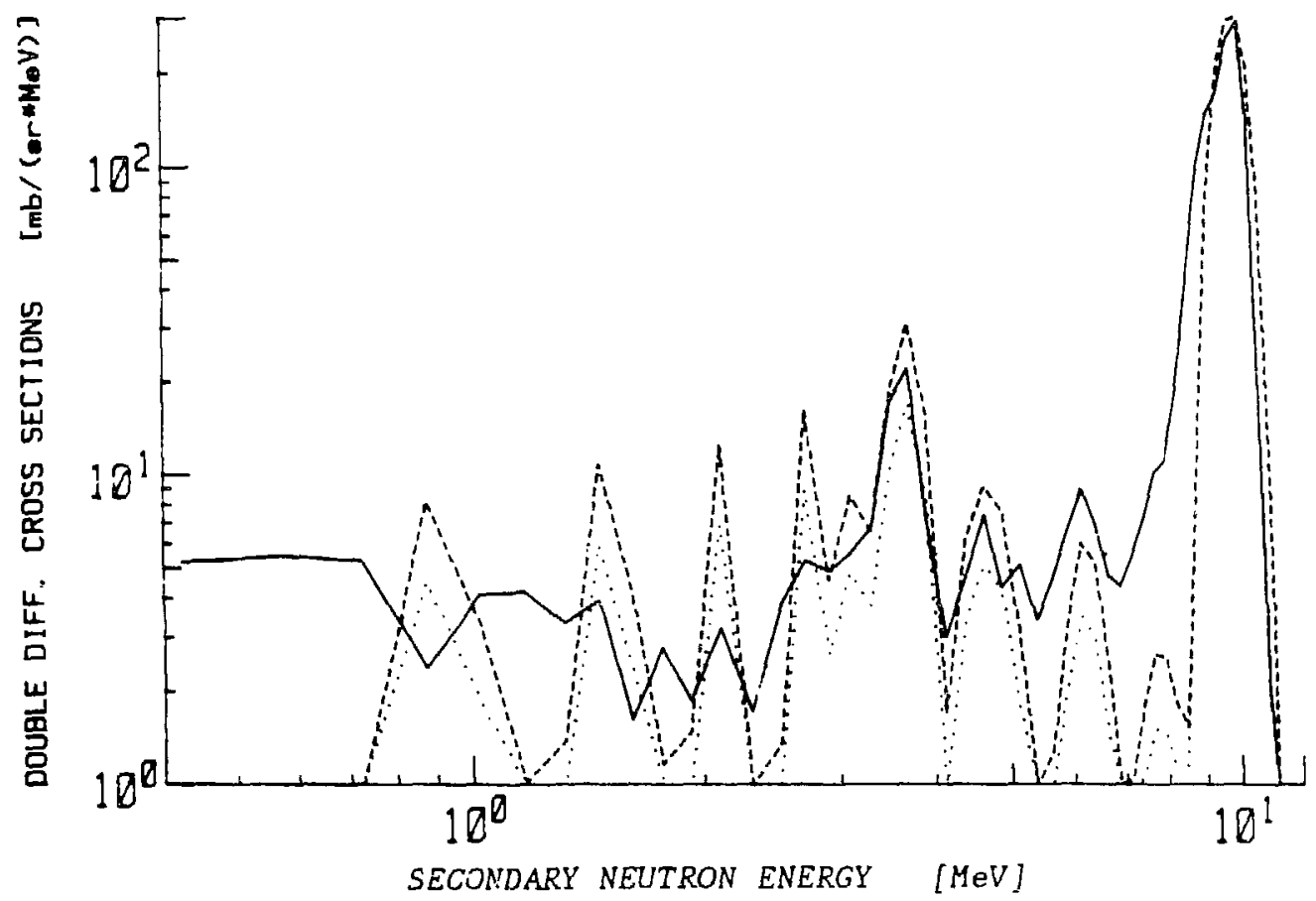

Fig. A-11. 10.0 MeV Neutrons on Boron-10 20.0 Deg.



Fig. A-12. $10.0 \mathrm{MeV}$ Neutrons on Boron-10 35.0 Deg. 


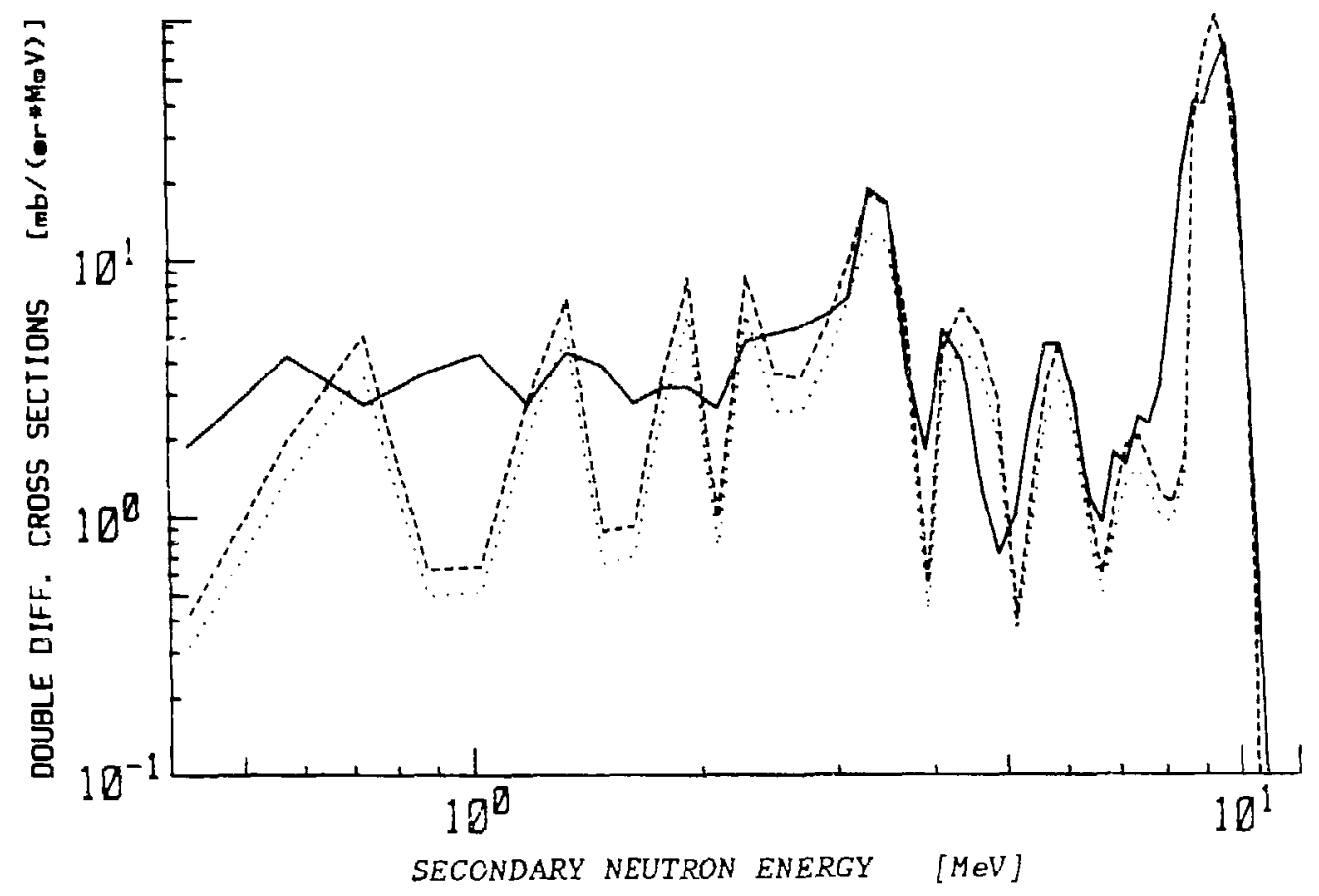

Fig. A-13. 10.0 MeV Neutrons on Boron-10 45.0 Deg.

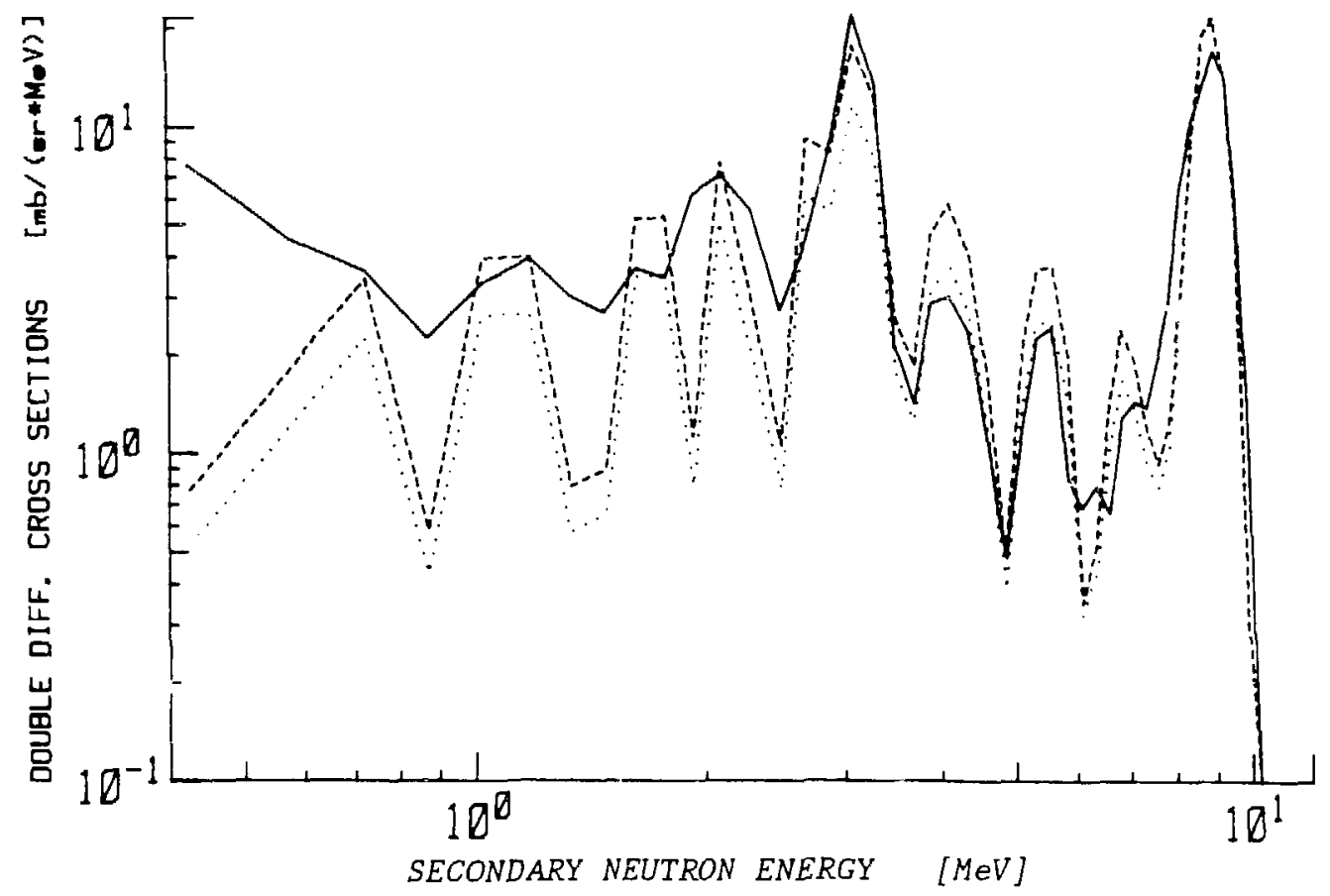

Fig. A-14. 10.0 MeV Neutrons or. Boron-1.0 60.0 Deg. 




Fig. A-15. 10.0 MeV Neutrons on Boron-10 75.0 Deg.

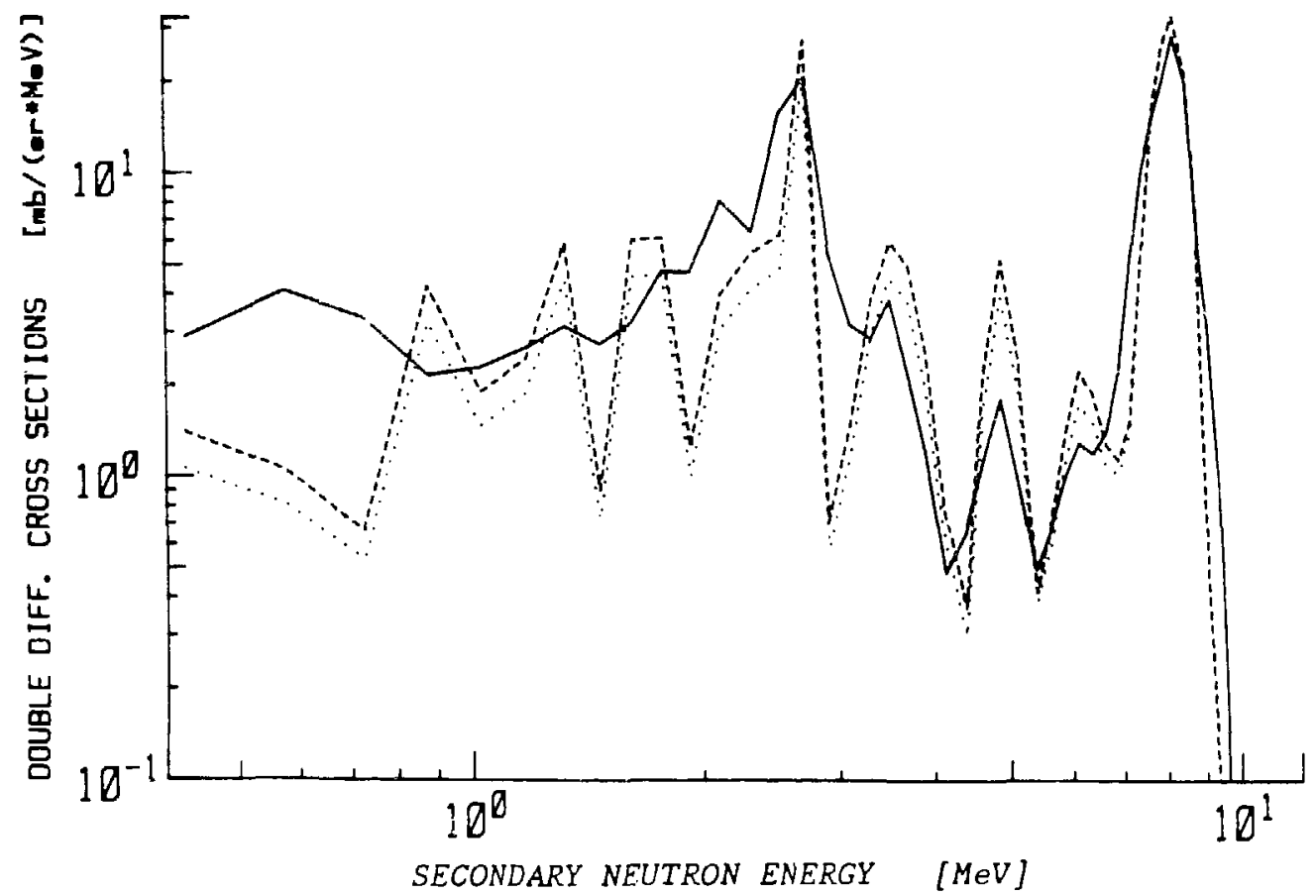

Fig. A-16. 10.0 MeV Neutrons on Boron-10 90.0 Deg. 


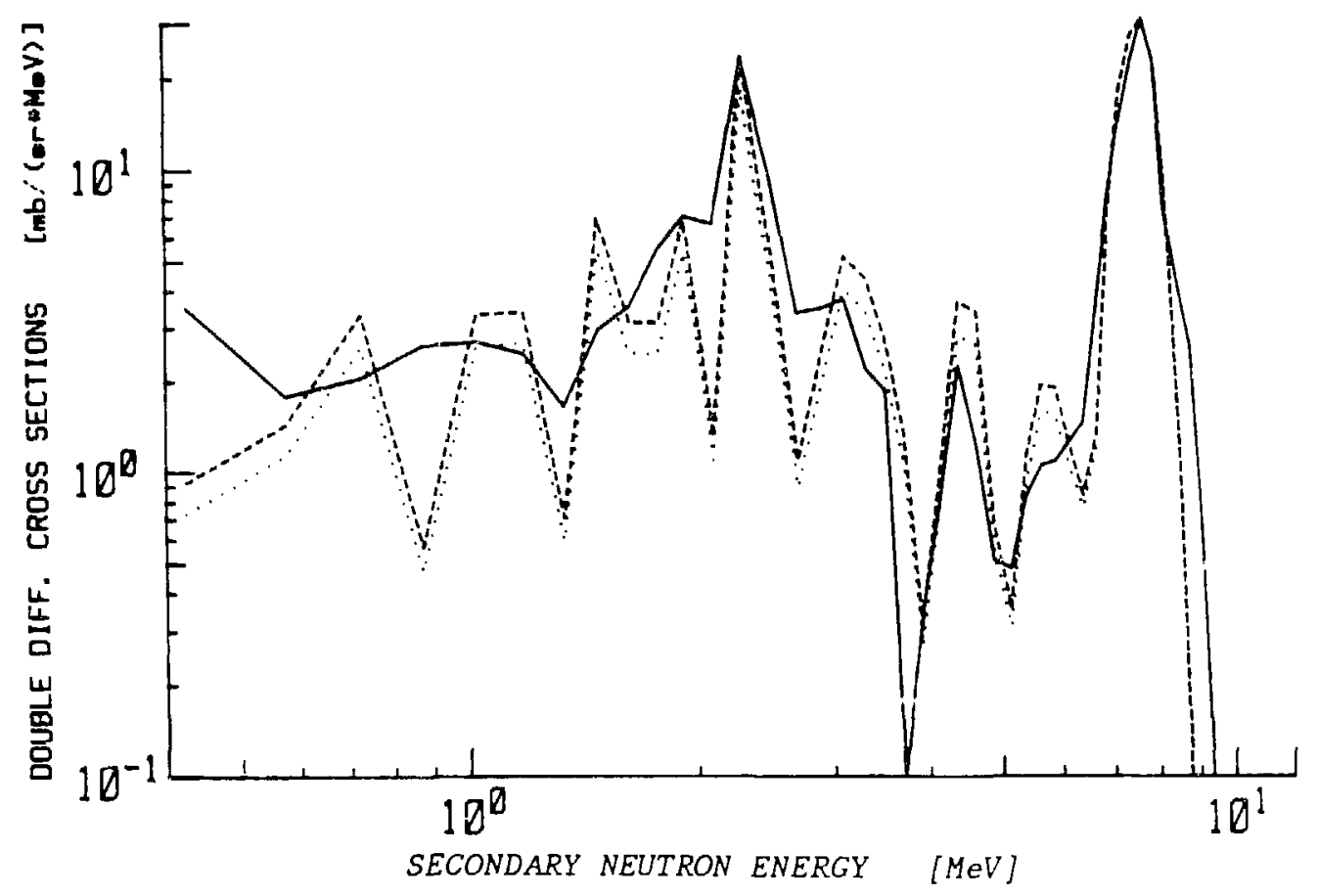

Fig. A-17. $10 \mathrm{MeV}$ Neutrons on Boron-10 $110.0 \mathrm{Deg}$.

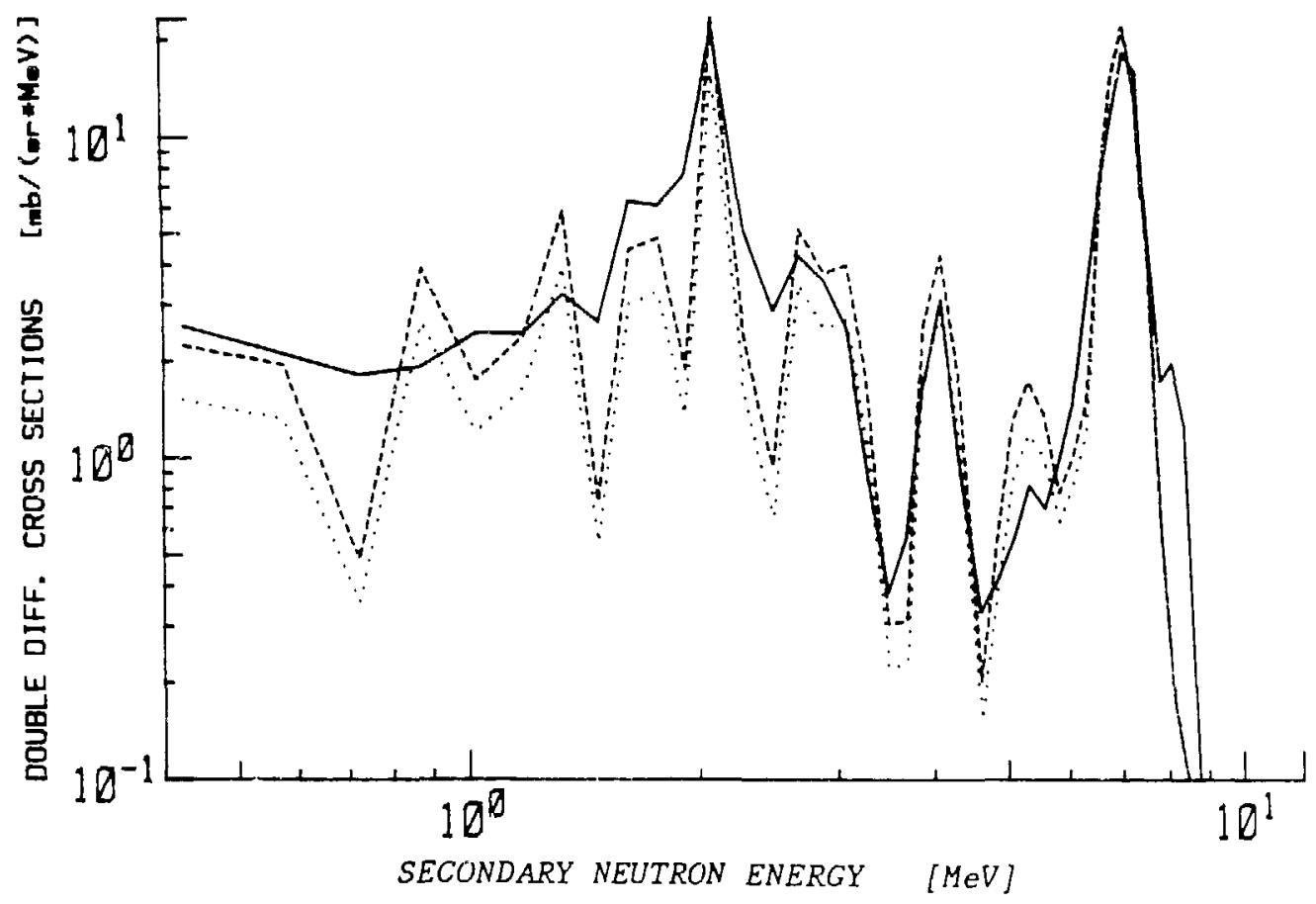

Fig. A. 18. 10.0 MeV Neutrons on Boron-10 130.0 Deg. 


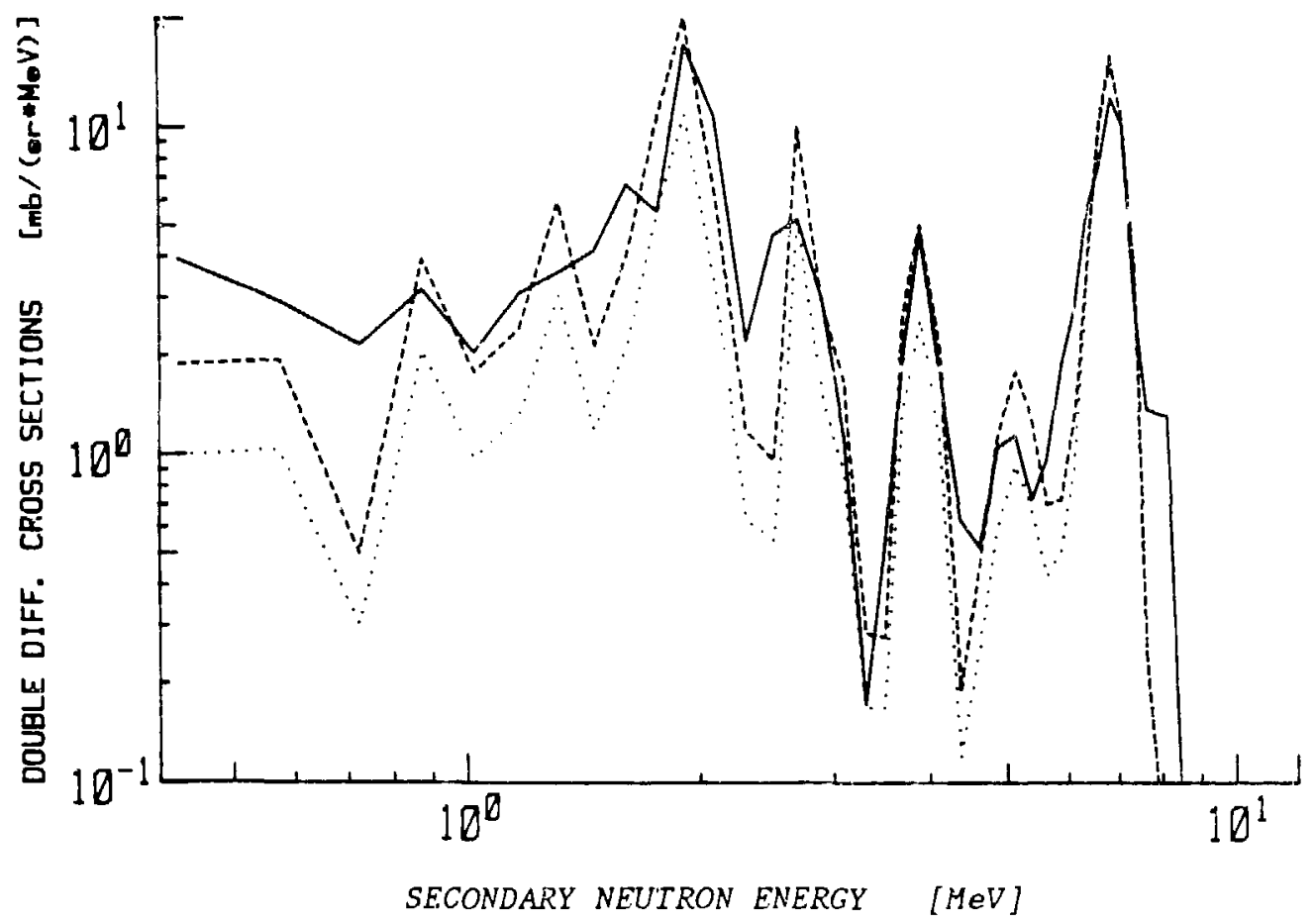

Fig. A-19. 10.0 MeV Neutrons on Boron-10 145.0 Deg. 


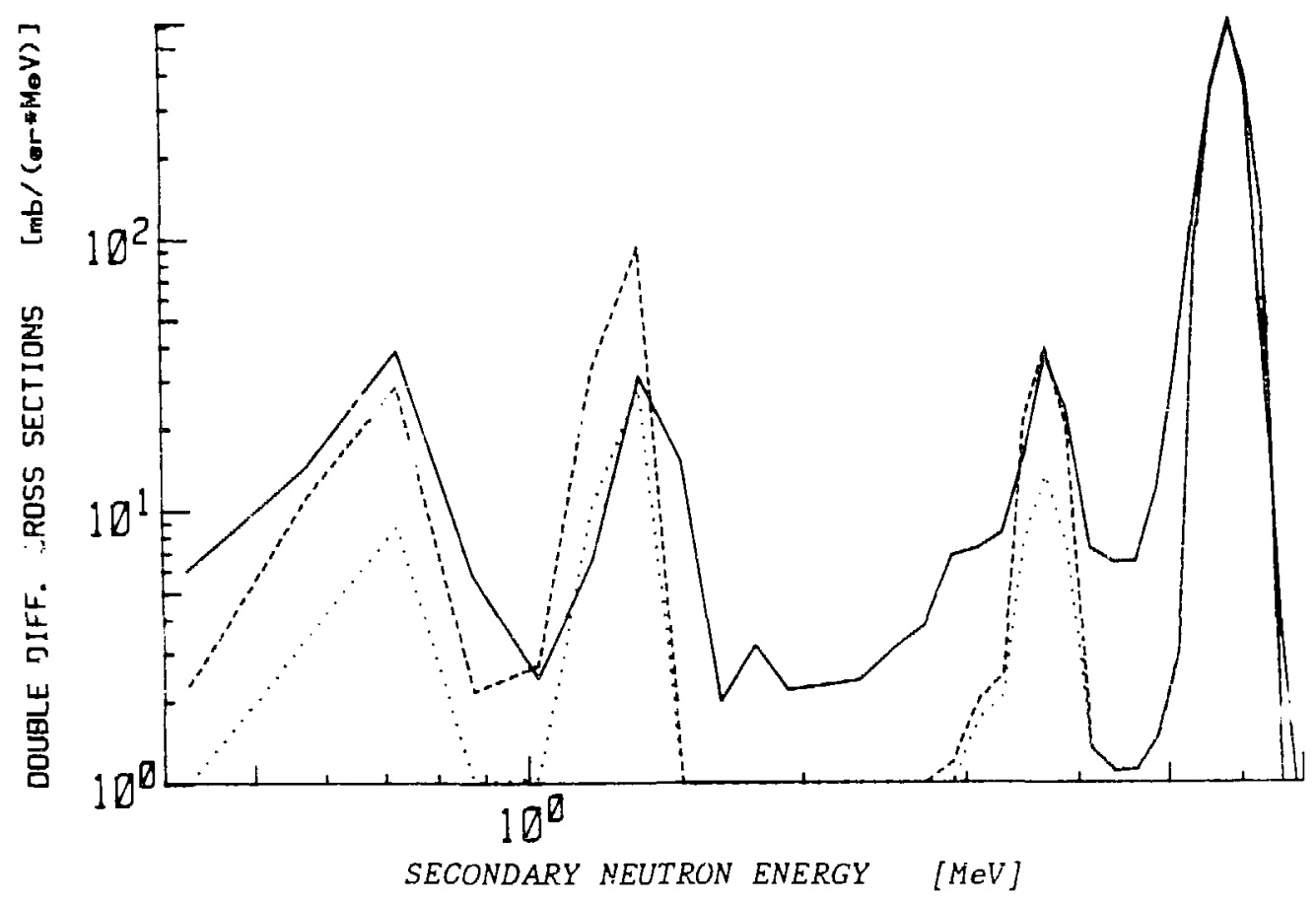

Fig. A-20. 6.0 MeV Neutrons on Boroll-11 20.0 Deg.



Fif. A-21. 6.0 MeV Neutrons on Boron-11 25.0 Deg. 


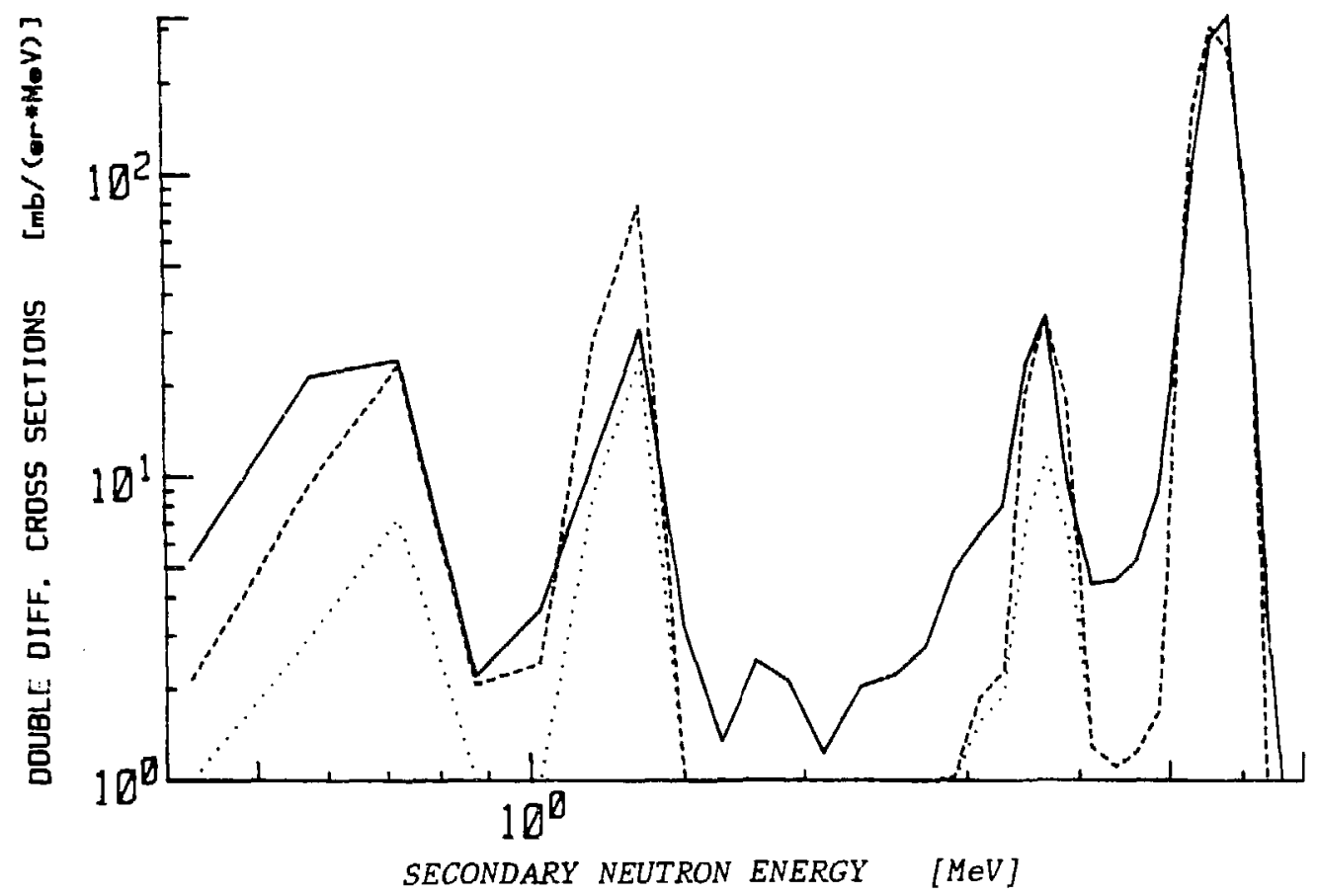

Fig. A-22. 6.0 MeV Neutrons on Boron-11 35.0 Deg.

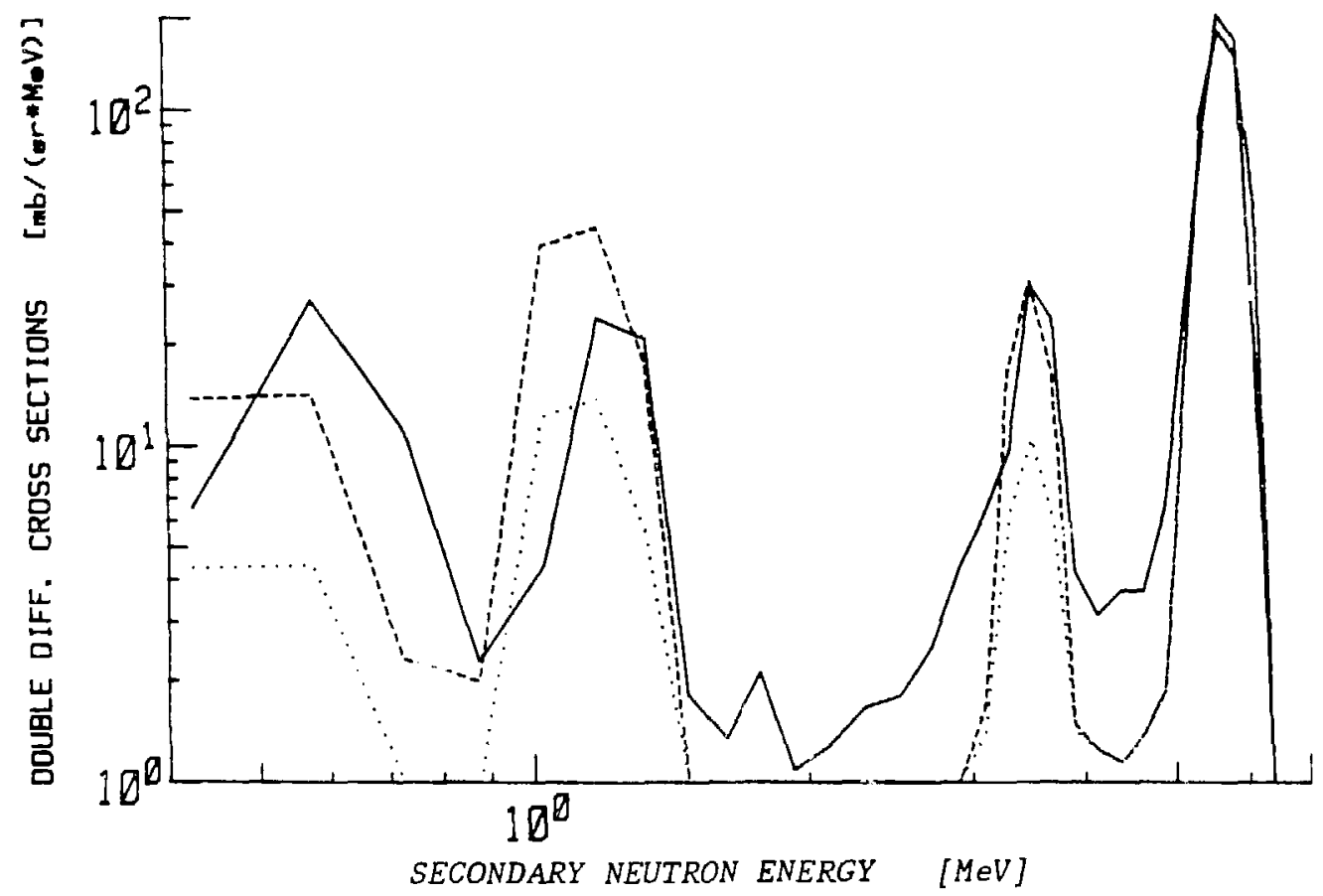

Fig. A-23. 6.0 MeV Neutrons on Boron-11 45.0 Deg. 


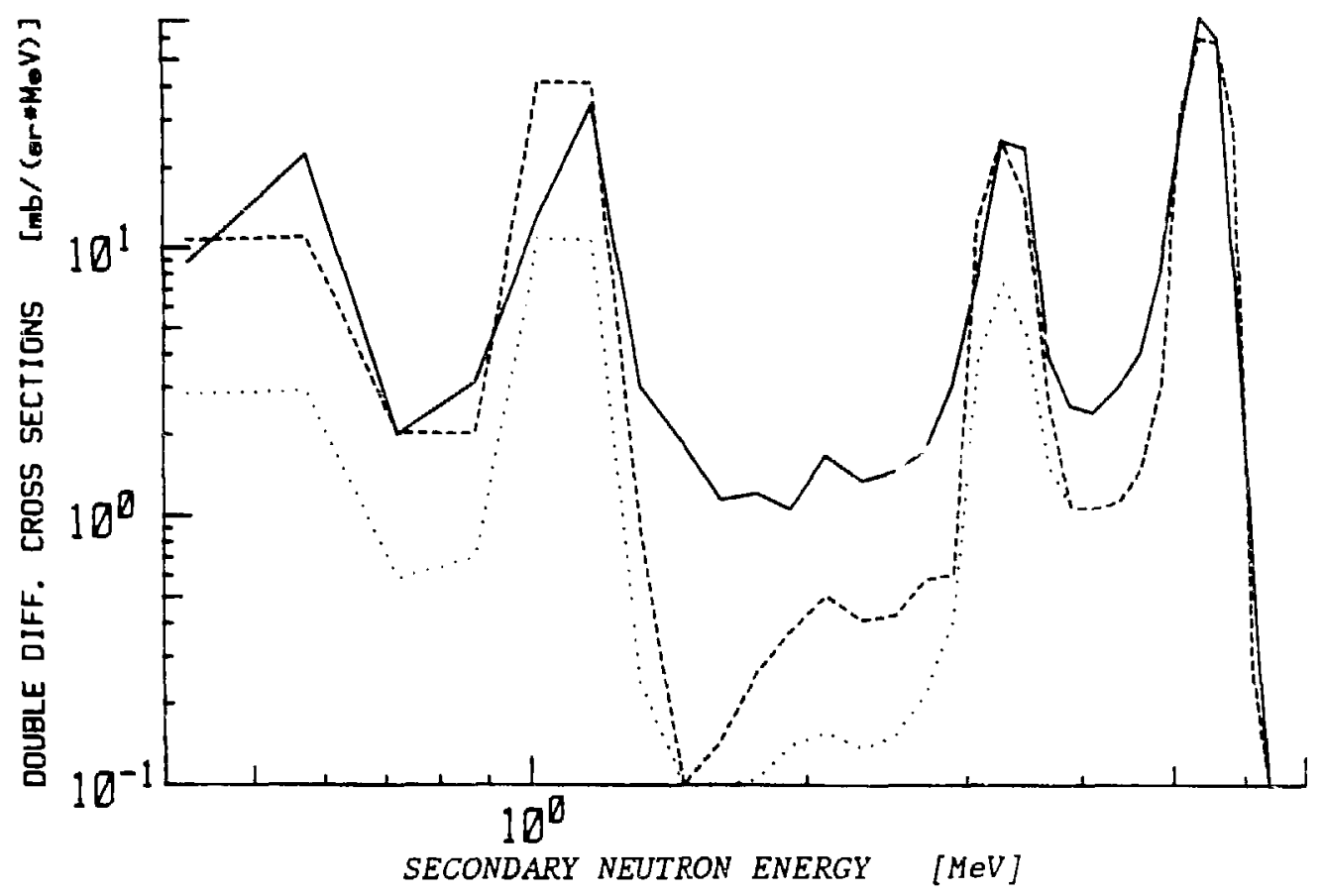

Fig. A-24. 6.0 MeV Neutrons on Boron-11 60.0 Deg.

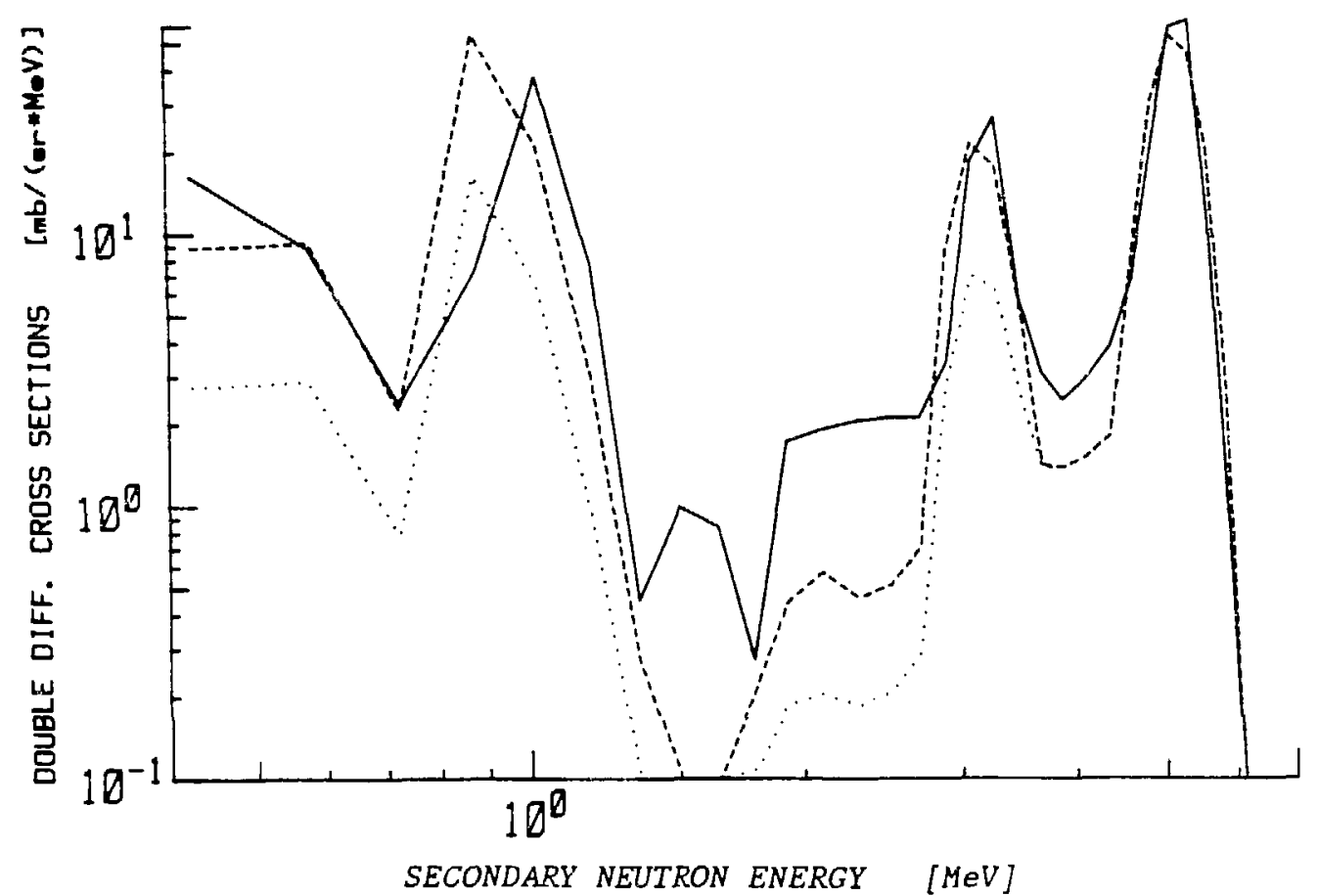

Fig. A-25. 6.0 MeV Neutrons on Boron-11 75.0 Deg. 




Fig. A-26. $6.0 \mathrm{MeV}$ Neutrons on Boron-11 $90.0 \mathrm{Deg}$.

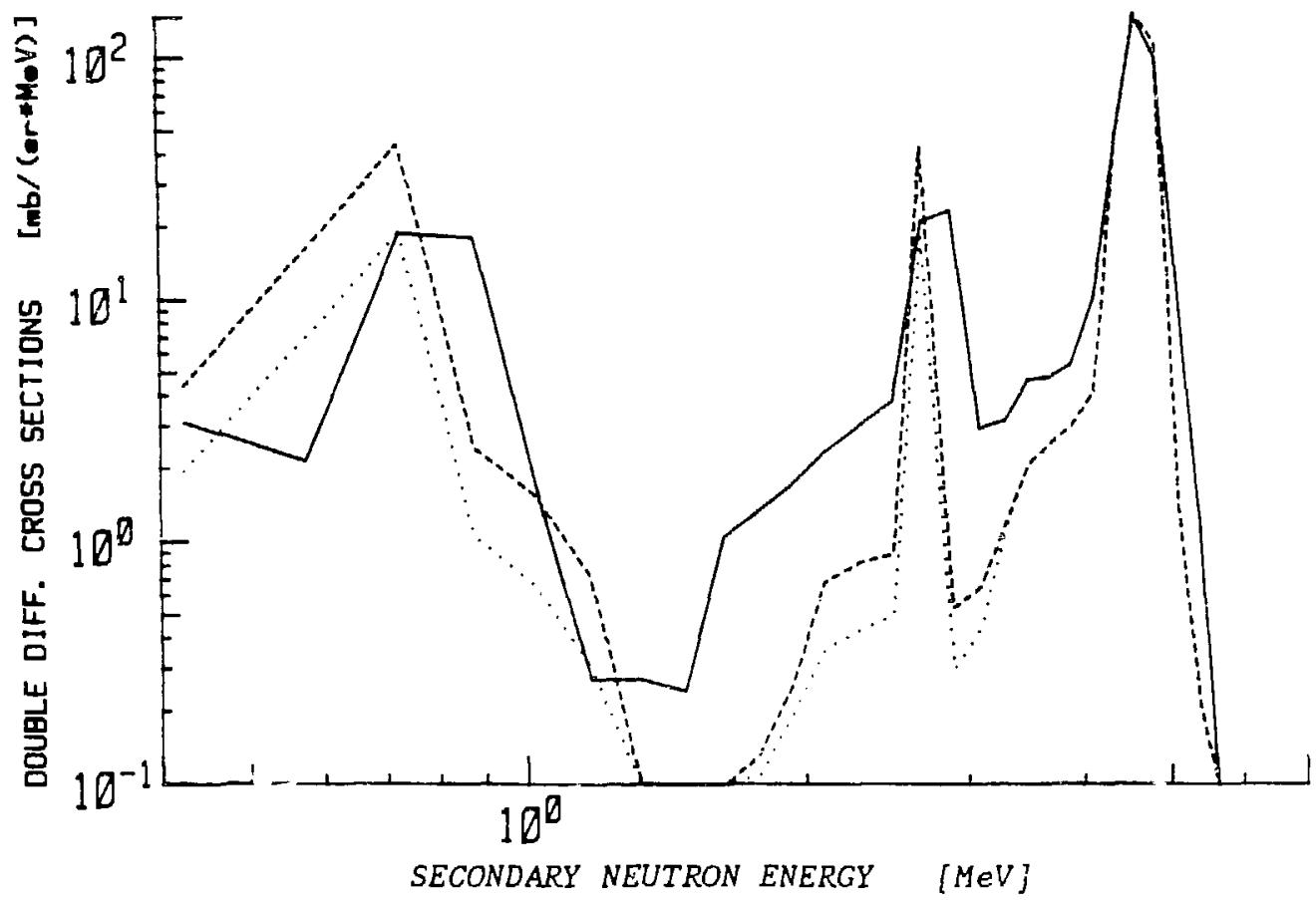

Fig. A-27. 6.0 MeV Neutrons on Boron-11 110.0 Deg. 


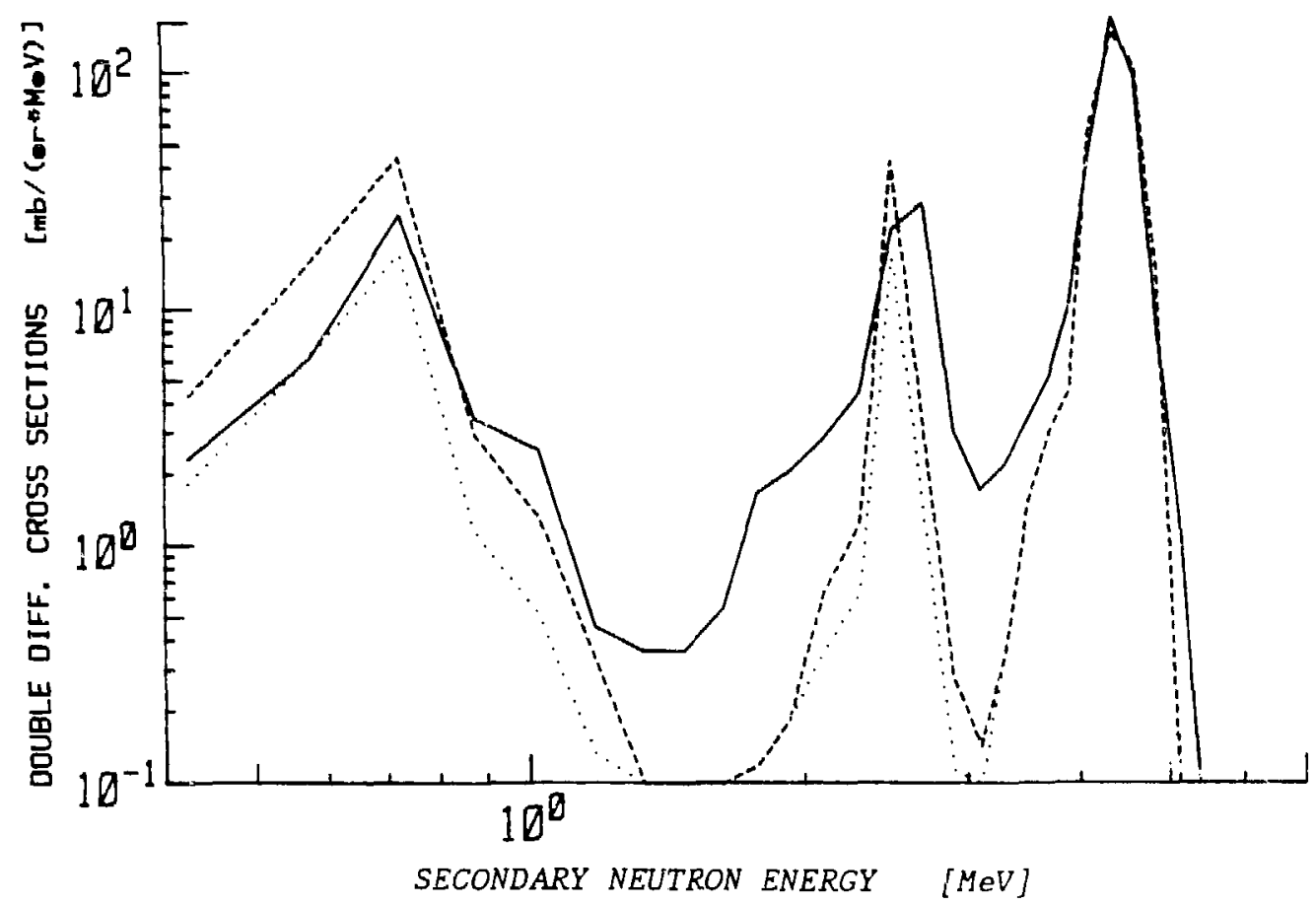

Fig. A-28. 6.0 MeV Neutrons on Boron-11 130.0 Deg.

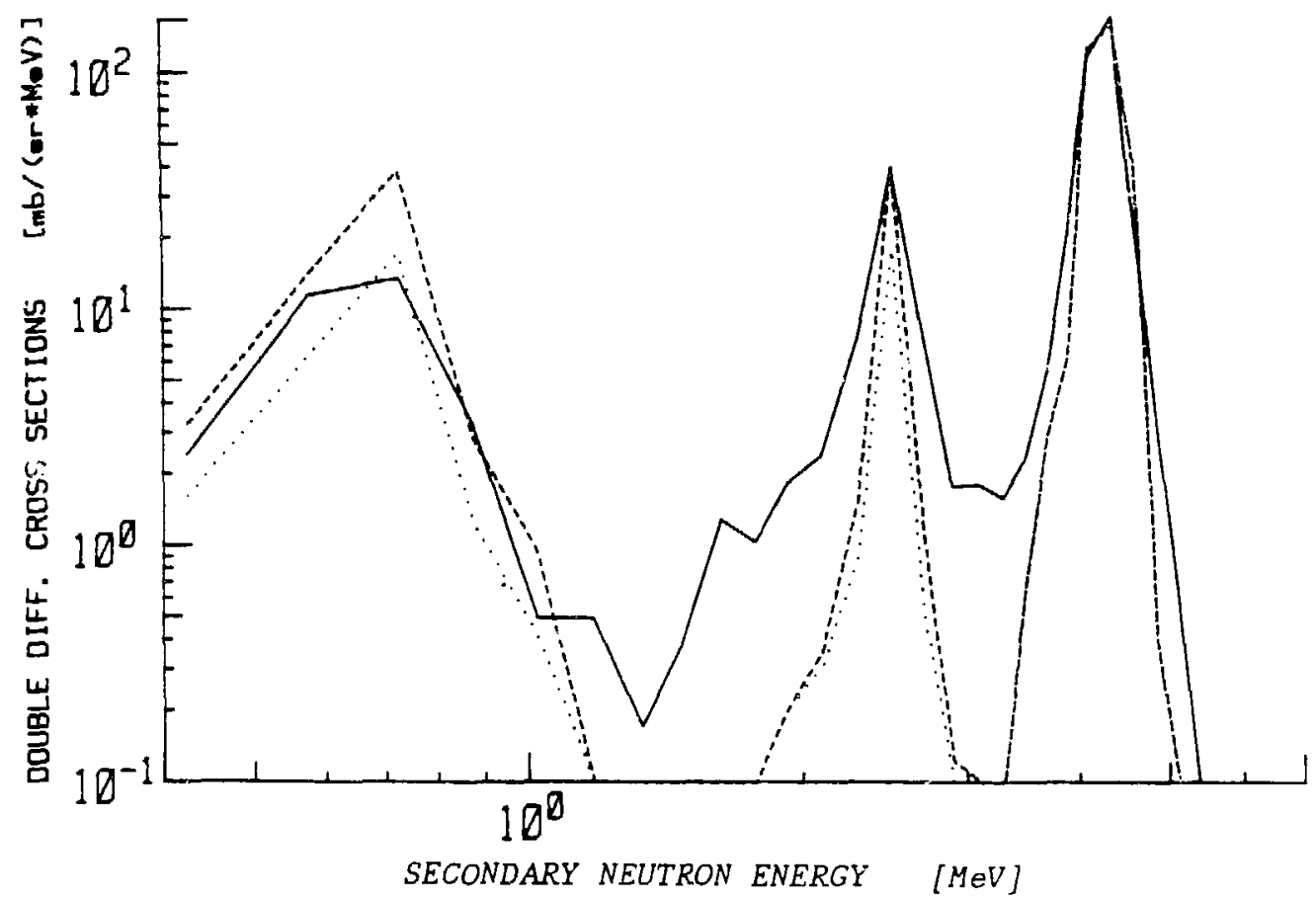

Fig. A-29. 6.0 MeV Neutrons on Boron-11 $145.0 \mathrm{Deg}$. 


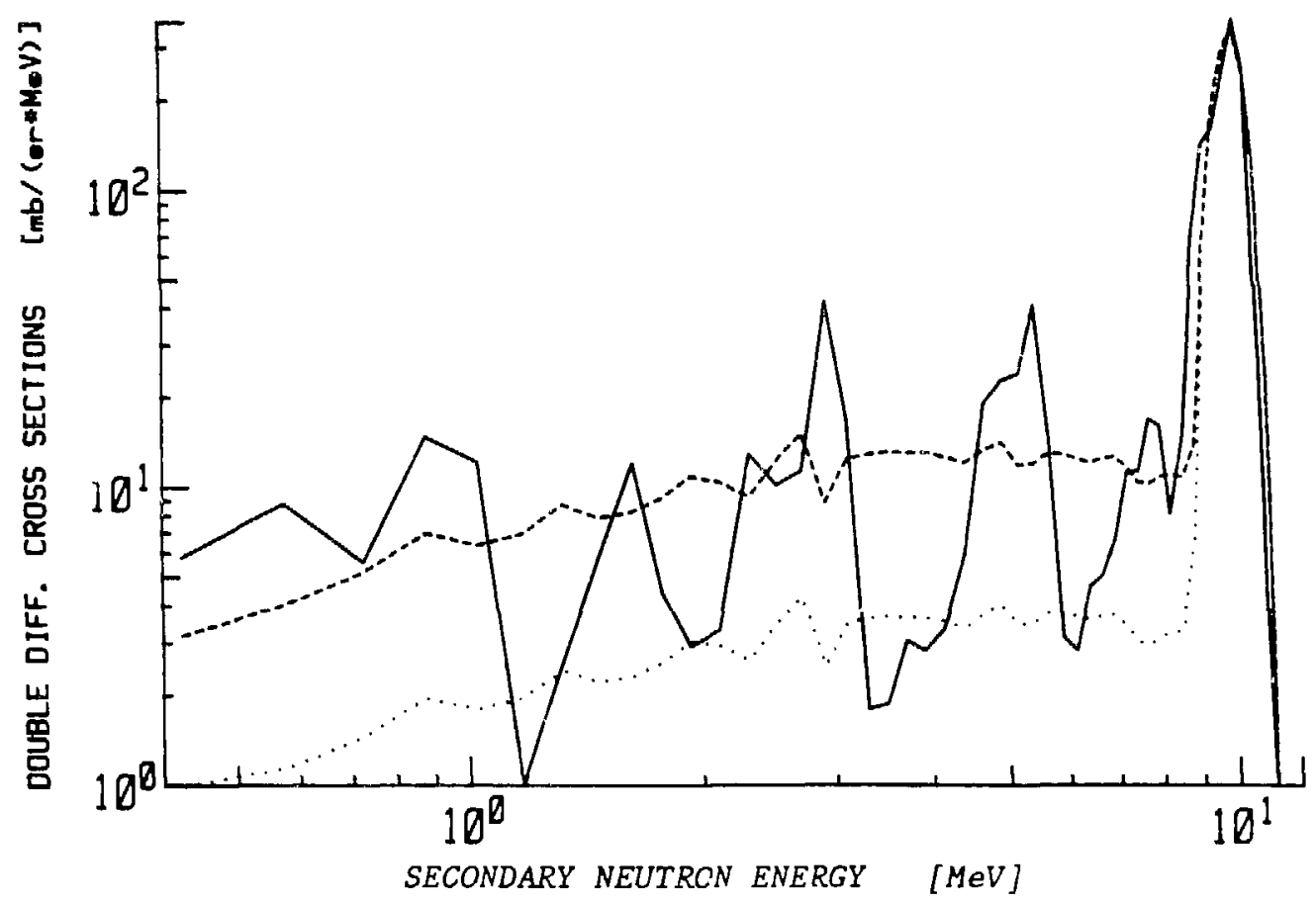

Fig. A-30. 10.0 MeV Neutrons on Boron-11 20.0 Deg.

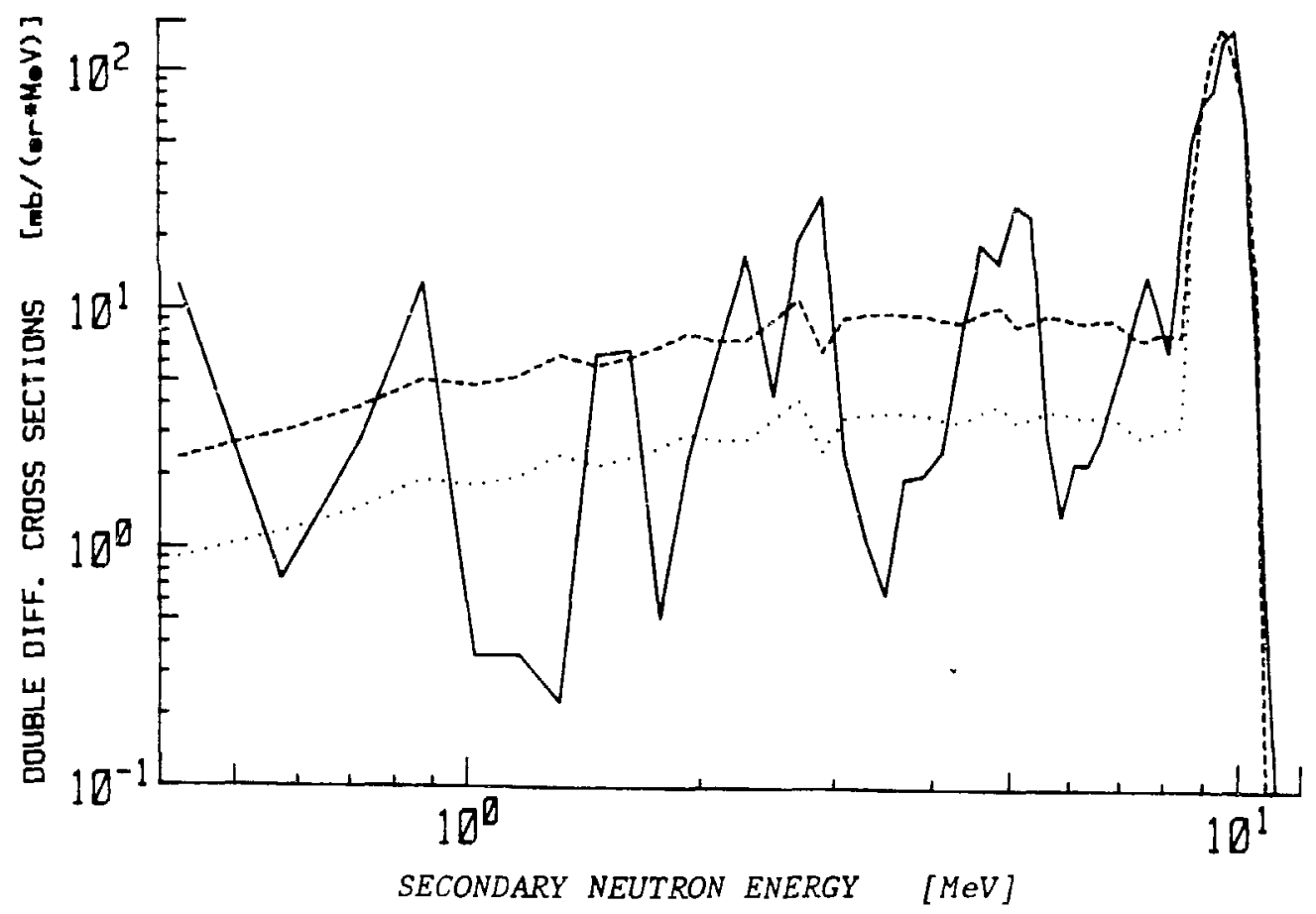

Fig. A-31. 10.0 MeV Neutrons on Boron-11 35.0 Deg. 




Fig. A-32. $10.0 \mathrm{MaV}$ Neutrons on Boron-11 45.0 Deg.

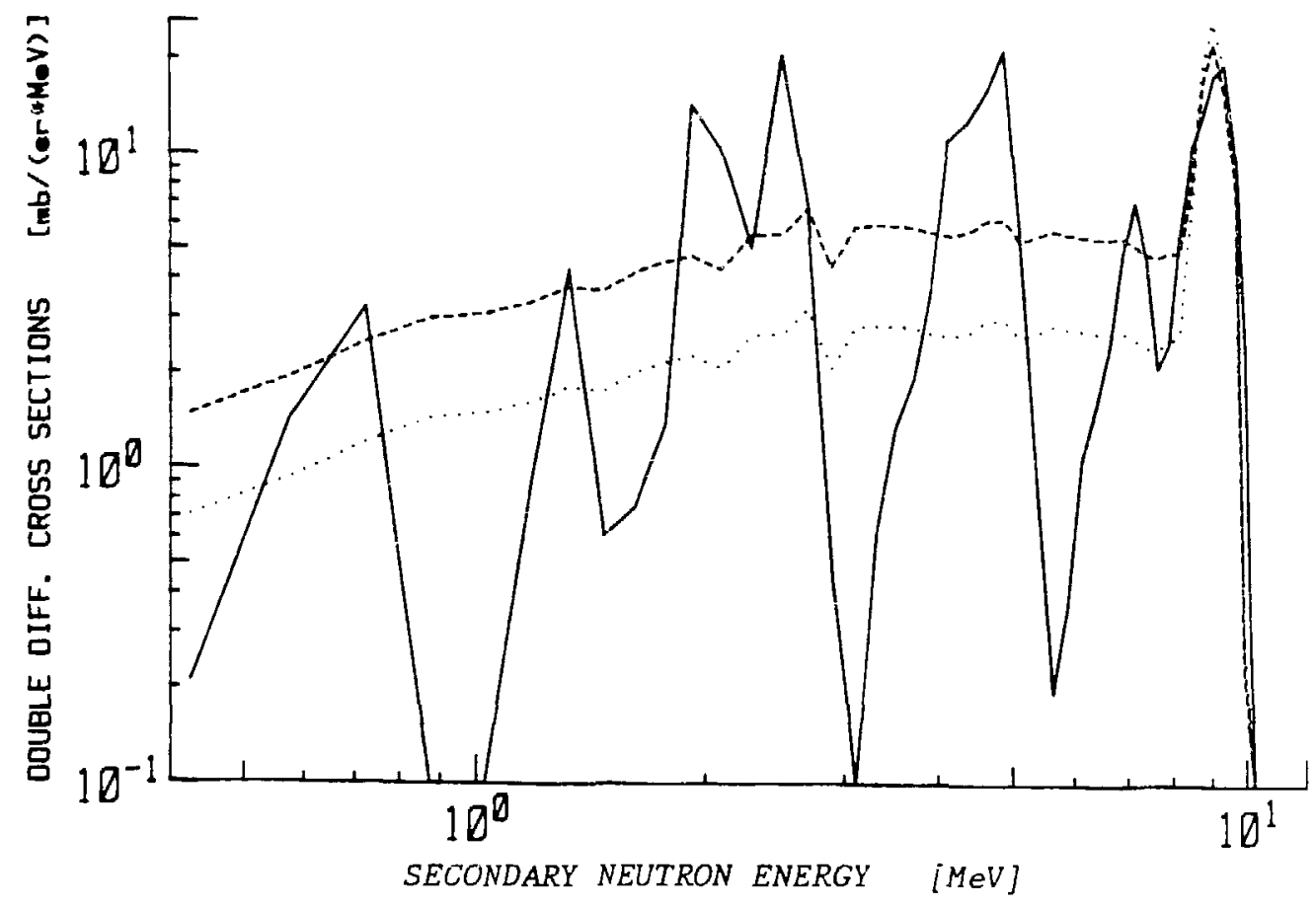

Fig. A-33. 10.0 MeV Neutrons on Boron-11 60.0 Deg. 


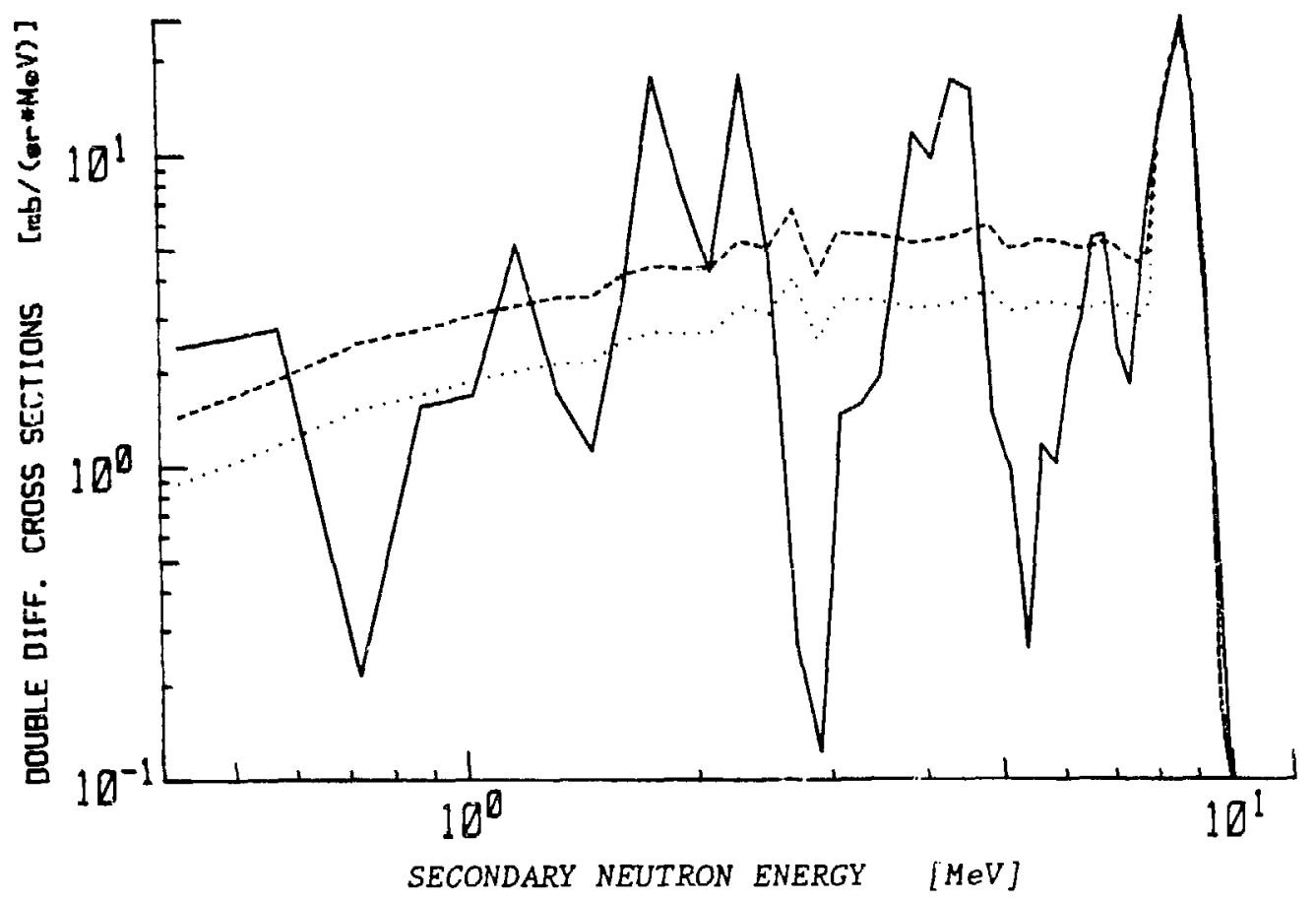

Fig. A-34 $10.0 \mathrm{MeV}$ Neutrons on Boron-11 $75.0 \mathrm{Deg}$.

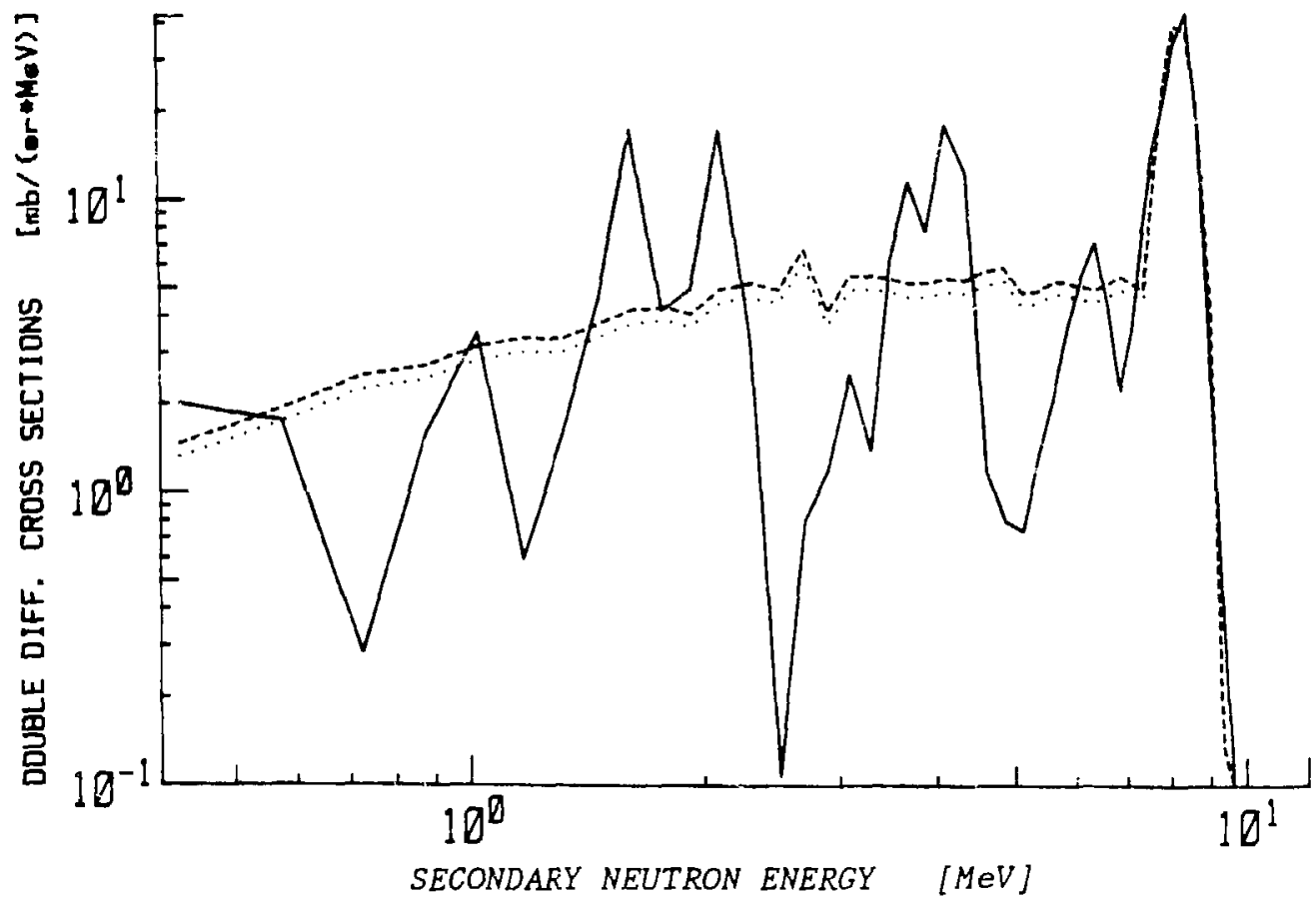

Fig. A-35. 10.0 MeV Neutrons on Boron-11 90.0 Deg. 


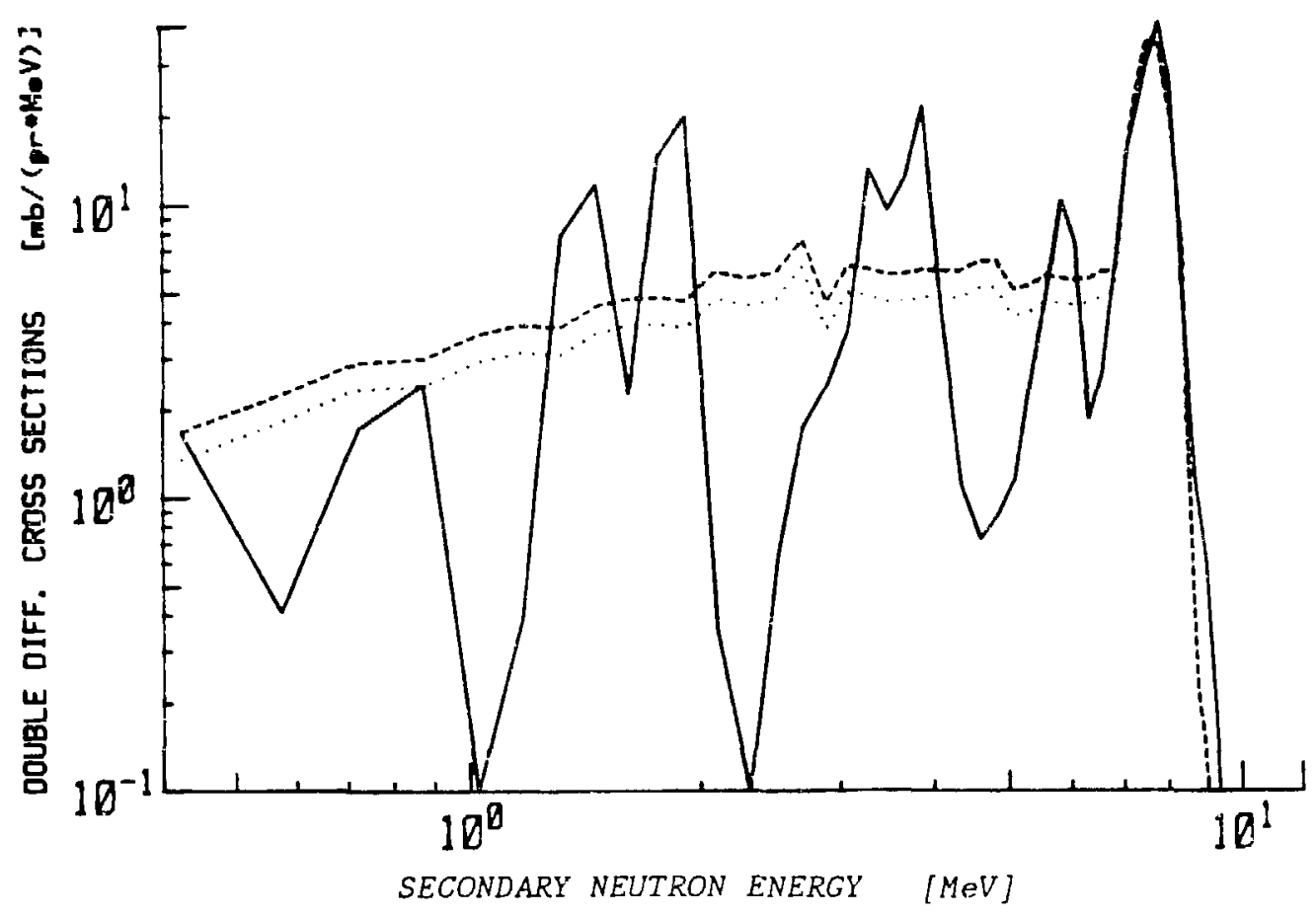

Fig. A-36. 10.0 MeV Neutrons on Boron-11 110.0 Deg.

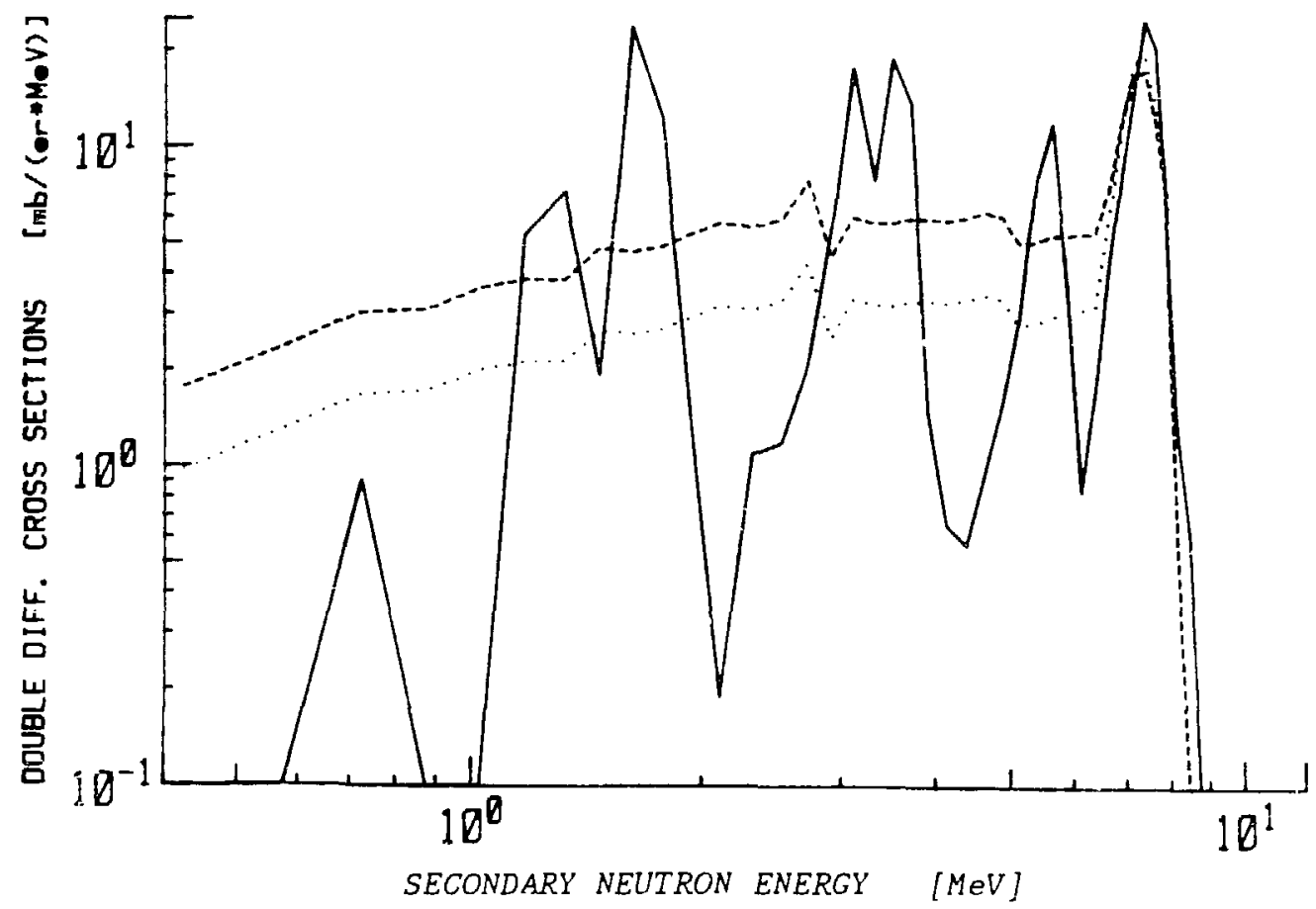

Fig. A-37. 10.0 MeV Neutrons on Boron-11 130.0 Deg. 


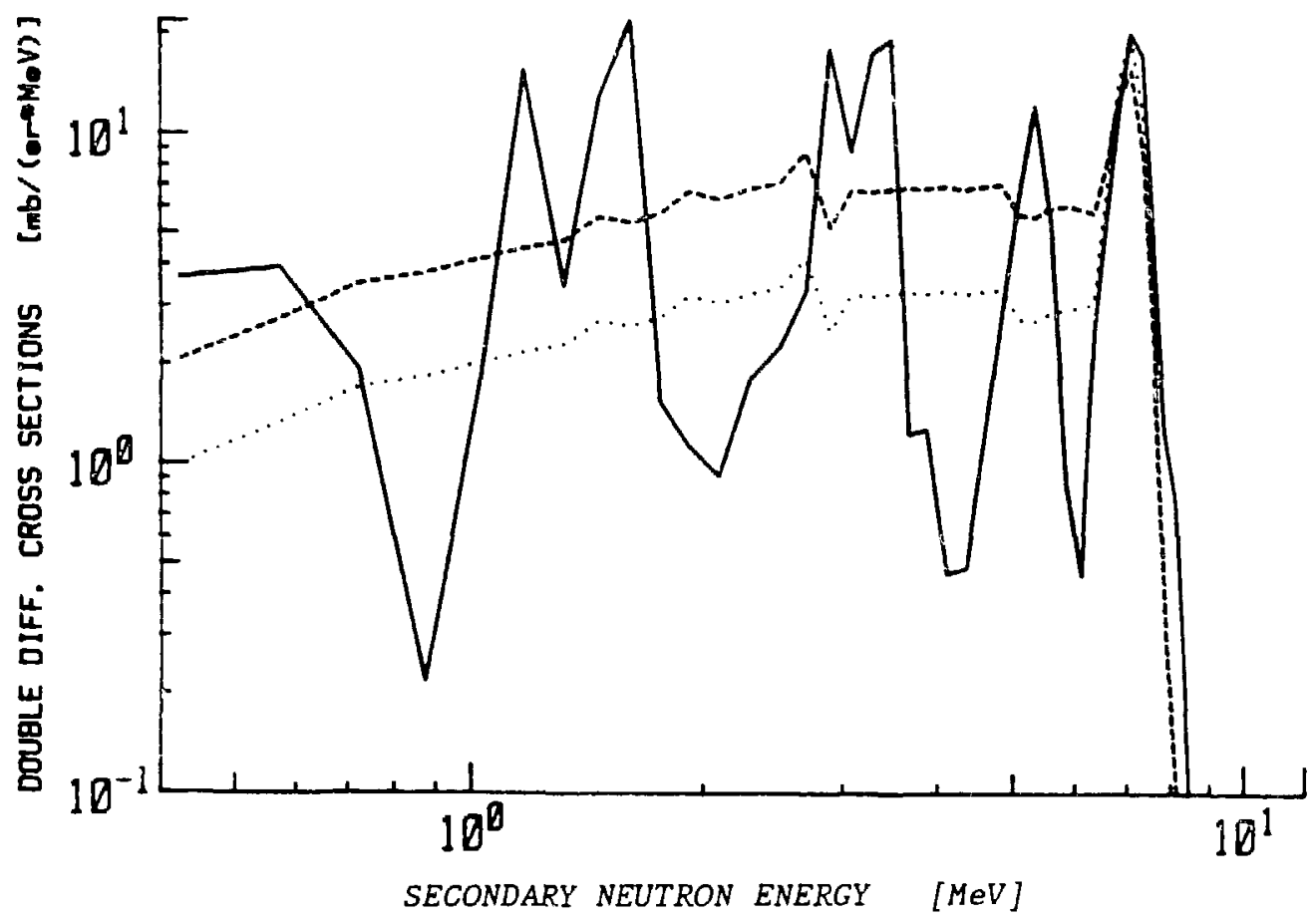

Fig. A-38. 10.0 MeV Neutrons on Boron-11 $145.0 \mathrm{Deg}$. 